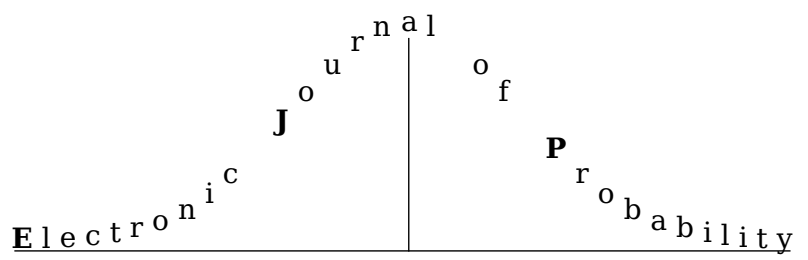

Electron. J. Probab. 26 (2021), article no. 37, 1-46.

ISSN: 1083-6489 https://doi.org/10.1214/21-EJP598

\title{
Scaling limits of crossing probabilities in metric graph GFF $^{*}$
}

\author{
Mingchang $\mathrm{Liu}^{\dagger} \quad \mathrm{Hao} \mathrm{Wu}^{\ddagger}$
}

\begin{abstract}
We consider metric graph Gaussian free field (GFF) defined on polygons of $\delta \mathbb{Z}^{2}$ with alternating boundary data. The crossing probabilities for level-set percolation of metric graph GFF have scaling limits. When the boundary data is well-chosen, the scaling limits of crossing probabilities can be explicitly constructed as "fusion" of multiple $\mathrm{SLE}_{4}$ pure partition functions.
\end{abstract}

Keywords: Gaussian free field; crossing probability; Schramm Loewner evolution.

MSC2020 subject classifications: 60G15; 60G60; 60J67.

Submitted to EJP on May 21, 2020, final version accepted on March 3, 2021.

\section{Introduction}

This article concerns crossing probability of level-set percolation of Gaussian free field (GFF) on the square lattice $\mathbb{Z}^{2}$. For $L>0$, consider the rectangle

$$
R_{L}=\{z: 0<\Re z<L, 0<\Im z<1\} .
$$

Let $y_{1}, y_{2}, y_{3}, y_{4}$ be its four corners, listed in counterclockwise order with $y_{2}=0$. For $\delta>0$, let $V_{\delta}=R_{L} \cap \delta \mathbb{Z}^{2}$ and let $y_{1}^{\delta}, y_{2}^{\delta}, y_{3}^{\delta}, y_{4}^{\delta}$ be its four corners, listed in counterclockwise order such that $y_{2}^{\delta}$ is closest to $y_{2}$. For two vertices $u, v \in \partial V_{\delta}$, we denote by (uv) the arc of $\partial V_{\delta}$ from $u$ to $v$ in counterclockwise order. Let $\Gamma^{\delta}$ be a discrete GFF (see Section 5.1) on $V_{\delta}$ with alternating boundary data:

$$
\mu \text { on }\left(y_{1}^{\delta} y_{2}^{\delta}\right) \cup\left(y_{3}^{\delta} y_{4}^{\delta}\right), \quad-\mu \text { on }\left(y_{2}^{\delta} y_{3}^{\delta}\right) \cup\left(y_{4}^{\delta} y_{1}^{\delta}\right),
$$

where $\mu>0$ is a positive constant. Let $\tilde{\Gamma}^{\delta}$ be the GFF on the metric graph $\tilde{V}_{\delta}$ (see Section 5.1) with the same boundary data. We are interested in the event that there

\footnotetext{
*Supported by Beijing Natural Science Foundation (JQ20001, Z180003) and Thousand Talents Plan for Young Professionals.

${ }^{\dagger}$ Tsinghua University, China. E-mail: liumc18@mails.tsinghua.edu.cn

${ }^{\ddagger}$ Tsinghua University, China. E-mail: hao.wu. proba@gmail.com
} 


\section{Crossing probabilities in metric graph GFF}

exists a path in $V_{\delta}$ (resp. $\tilde{V}_{\delta}$ ) from $\left(y_{1}^{\delta} y_{2}^{\delta}\right)$ to $\left(y_{3}^{\delta} y_{4}^{\delta}\right)$ such that $\Gamma^{\delta}$ (resp. $\tilde{\Gamma}^{\delta}$ ) is non-negative on this path. We denote this event by

$$
\left\{\left(y_{1}^{\delta} y_{2}^{\delta}\right) \stackrel{\Gamma^{\delta} \geq 0}{\longleftrightarrow}\left(y_{3}^{\delta} y_{4}^{\delta}\right)\right\} \quad \text { and } \quad\left\{\left(y_{1}^{\delta} y_{2}^{\delta}\right) \stackrel{\tilde{\Gamma}^{\delta} \geq 0}{\longleftrightarrow}\left(y_{3}^{\delta} y_{4}^{\delta}\right)\right\}
$$

for $\Gamma^{\delta}$ and $\tilde{\Gamma}^{\delta}$ respectively. Although, both discrete GFF $\Gamma^{\delta}$ and metric graph GFF $\tilde{\Gamma}^{\delta}$ converge as distributions to the continuum GFF as $\delta \rightarrow 0$, the probabilities for such crossing events have distinct scaling limits, as proved in [DWW20, Theorem 1.2]. It is then natural to ask whether we are able to give explicit formula for scaling limits of such crossing probabilities.

The answer to this question relies on Schramm-Sheffield's famous work on level lines of GFF. We call $(\Omega ; x, y)$ a Dobrushin domain if $\Omega \subsetneq \mathbb{C}$ is non-empty simply connected and $x, y$ are distinct boundary points. In [SS09], the authors prove that there exists $\lambda=\lambda\left(\mathbb{Z}^{2}\right)>0$ such that the zero level line of discrete GFF on Dobrushin domains of $\delta \mathbb{Z}^{2}$ with boundary data $\pm \lambda$ converges in distribution to Schramm-Loewner Evolution ( $\mathrm{SLE}_{4}$, see Section 2.3). Based on this result, one is able to show that [DWW20, Theorem 1.3], when $\mu=\lambda$,

$$
\lim _{\delta \rightarrow 0} \mathbb{P}\left[\left(y_{1}^{\delta} y_{2}^{\delta}\right) \stackrel{\Gamma^{\delta} \geq 0}{\longleftrightarrow}\left(y_{3}^{\delta} y_{4}^{\delta}\right)\right]=q
$$

where $q$ is the cross-ratio of the rectangle: let $\varphi$ be any conformal map from $R_{L}$ onto the upper-half plane $\mathbb{H}$ with $\varphi\left(y_{1}\right)<\varphi\left(y_{2}\right)<\varphi\left(y_{3}\right)<\varphi\left(y_{4}\right)$, then

$$
q=\frac{\left(\varphi\left(y_{2}\right)-\varphi\left(y_{1}\right)\right)\left(\varphi\left(y_{4}\right)-\varphi\left(y_{3}\right)\right)}{\left(\varphi\left(y_{3}\right)-\varphi\left(y_{1}\right)\right)\left(\varphi\left(y_{4}\right)-\varphi\left(y_{2}\right)\right)} .
$$

This gives answer to the case of discrete GFF. The authors in [DWW20] derive (1.1) by showing that the scaling limit of the crossing probability in discrete GFF is the same as the one for continuum GFF whose crossing probability is calculated in [PW19, Theorem 1.4]. Such probability is also calculated in [KW11]. It remains to answer the question for the case of metric graph GFF.

The goal of this article is to derive explicit formula for scaling limits of crossing probability in metric graph GFF. We will show that, when $\mu=2 \lambda$,

$$
\lim _{\delta \rightarrow 0} \mathbb{P}\left[\left(y_{1}^{\delta} y_{2}^{\delta}\right) \stackrel{\tilde{\Gamma}^{\delta} \geq 0}{\longleftrightarrow}\left(y_{3}^{\delta} y_{4}^{\delta}\right)\right]=q^{4},
$$

where $q$ is the cross-ratio of the rectangle as in (1.2). In fact, we are able to give answer in a more general setting: we can calculate the scaling limits of crossing probabilities for the metric graph GFF with alternating boundary data on a polygon with $2 N$ marked points on the boundary. To state our main result, we first introduce some notations about planar link patterns.

For $p \in \mathbb{Z}_{>0}$, we call $\left(\Omega ; x_{1}, \ldots, x_{p}\right)$ a polygon if $\Omega \nsubseteq \mathbb{C}$ is non-empty simply connected and $x_{1}, \ldots, x_{p}$ are $p$ boundary points in counterclockwise order lying on locally connected boundary segments. We first introduce planar pair partitions. Suppose $p=2 N$ is even and suppose there are $N$ non-intersecting simple curves in $\Omega$ connecting the $2 N$ boundary points pairwise. These $N$ curves form a planar pair partition that we denote by $\alpha=\left\{\left\{a_{1}, b_{1}\right\}, \ldots\left\{a_{N}, b_{N}\right\}\right\}$ with $\left\{a_{1}, b_{1}, \ldots, a_{N}, b_{N}\right\}=\{1,2, \ldots, 2 N\}$. We call the pairs $\{a, b\}$ in $\alpha$ links. We denote by $\mathrm{PP}_{\mathrm{N}}$ the set of planar pair partitions with $2 N$ points and set $\mathrm{PP}=\bigsqcup_{\mathrm{N} \geq 0} \mathrm{PP}_{\mathrm{N}}$.

Next, we introduce general planar link patterns. The planar pair partitions then arise as a special case. Suppose $\left(\Omega ; x_{1}, \ldots, x_{p}\right)$ is a polygon. Fix a multiindex $\varsigma=\left(s_{1}, \ldots, s_{p}\right) \in$ 
$\mathbb{Z}_{>0}^{p}$ such that $\sum_{i=1}^{p} s_{i}$ is even and denote by

$$
\ell=\frac{1}{2} \sum_{i=1}^{p} s_{i} \in \mathbb{Z}_{>0}
$$

Suppose there are $\ell$ simple curves in $\Omega$ connecting the $p$ boundary points pairwise such that they do not intersect except at their common end points. These $\ell$ curves form a planar link pattern that we call planar $\ell$-link patterns of $p$ points. Precisely, we call planar $\ell$-link patterns of $p$ points with index valences $\varsigma=\left(s_{1}, \ldots, s_{p}\right)$ as collections $\omega=\left\{\left\{a_{1}, b_{1}\right\}, \ldots,\left\{a_{\ell}, b_{\ell}\right\}\right\}$ of $\ell$-links $\{a, b\}$ which connect a pair of distinct indices $a, b \in$ $\{1,2, \ldots, p\}$ such that, for any $i \in\{1,2, \ldots, p\}$, the index $i$ is an endpoint of exactly $s_{i}$ links and that none of the links intersect except at their common endpoints. We denote the collection of $\ell$-link patterns of $p$ points with index valences $\varsigma$ by $\operatorname{LP}_{\varsigma}$. With such definition, when $p=2 N$ is even, the planar $N$-link pattern of $2 N$ points with index valences $\varsigma=(1, \ldots, 1)$ is a planar pair partition and $\mathrm{LP}_{(1, \ldots, 1)}=\mathrm{PP}_{\mathrm{N}}$.

In this article, we are interested in planar $2 N$-link patterns of $2 N$ points with index valences $\varsigma=(2, \ldots, 2)$, see Figure 1 for $N=2$. With the above definition, the collection of such planar link patterns is denoted by $\mathrm{LP}_{(2, \ldots, 2)}$ where the index has length $2 N$.
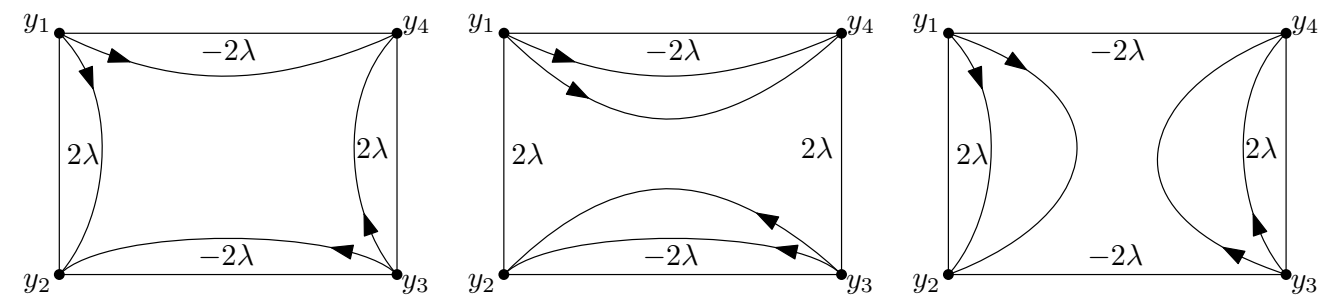

Figure 1: Consider metric graph GFF in rectangle with alternating boundary data when $\mu=2 \lambda$. Consider the positive first passage sets attached to $\left(y_{1}^{\delta} y_{2}^{\delta}\right)$ and to $\left(y_{3}^{\delta} y_{4}^{\delta}\right)$ and consider the negative first passage sets attached to $\left(y_{2}^{\delta} y_{3}^{\delta}\right)$ and to $\left(y_{4}^{\delta} y_{1}^{\delta}\right)$. Their frontier form a planar 4-link pattern of 4 points with index valences $\varsigma=(2,2,2,2)$. There are three possibilities as indicated in the figure. In the right panel, there is negative vertical crossing of the metric graph GFF. In the middle panel, there is positive horizontal crossing. In the left panel, there is neither positive horizontal crossing nor negative vertical crossing. As $\delta \rightarrow 0$, the frontier converges to level lines of continuum GFF with the same boundary data and the four level lines are as follows: there are two level lines starting from $y_{1}$ (resp. from $y_{3}$ ), one has height $-\lambda$ and the other one has height $\lambda$.

Fix a polygon $\left(\Omega ; y_{1}, \ldots, y_{2 N}\right)$ such that $\Omega \subset[-C, C]^{2}$ for some $C>0$. Suppose $\left\{\left(\Omega^{\delta} ; y_{1}^{\delta}, \ldots, y_{2 N}^{\delta}\right)\right\}_{\delta>0}$ are polygons such that $\Omega^{\delta} \subset[-C, C]^{2}$ for all $\delta>0$. Suppose $\left(\Omega^{\delta} ; y_{1}^{\delta}, \ldots, y_{2 N}^{\delta}\right)$ converges to $\left(\Omega ; y_{1}, \ldots, y_{2 N}\right)$ as $\delta \rightarrow 0$ in the following sense:

$$
\begin{aligned}
& {[-C, C]^{2} \backslash \Omega^{\delta} \text { converges to }[-C, C]^{2} \backslash \Omega \text { in Hausdorff metric }} \\
& \text { and } y_{i}^{\delta} \rightarrow y_{i} \text { for each } 1 \leq i \leq 2 N .
\end{aligned}
$$

Consider metric graph GFF $\tilde{\Gamma}^{\delta}$ in $\left(\Omega^{\delta} ; y_{1}^{\delta}, \ldots, y_{2 N}^{\delta}\right)$ with alternating boundary data:

$$
2 \lambda \text { on }\left(y_{2 j-1}^{\delta} y_{2 j}^{\delta}\right), \quad \text { and } \quad-2 \lambda \text { on }\left(y_{2 j}^{\delta} y_{2 j+1}^{\delta}\right), \quad \text { for } j \in\{1, \ldots, N\} \text {, }
$$

where $y_{2 N+1}=y_{1}$ by convention. Consider positive first passage set (see Section 5.2) of $\tilde{\Gamma}^{\delta}$ attached to the boundary segments $\left(y_{2 j-1}^{\delta} y_{2 j}^{\delta}\right)$, and negative first passage set attached to the boundary segments $\left(y_{2 j}^{\delta} y_{2 j+1}^{\delta}\right)$, for $j \in\{1, \ldots, N\}$. The frontier of these 
first passage sets is a collection of $2 N$ curves connecting the $2 N$ boundary points so that their end points form a planar $2 N$-link pattern of $2 N$ points with index valences $\varsigma=(2,2, \ldots, 2)$. See Figure 1 . We denote the link pattern by $\mathcal{A}^{\delta}$. Our main result is the following.

Theorem 1.1. Fix $N \geq 1$ and the index valences $\varsigma=(2, \ldots, 2)$ of length $2 N$. Consider the frontier of first passage sets of metric graph GFF in $\Omega^{\delta}$ with alternating boundary data (1.5). The frontier is a collection of $2 N$ curves connecting the $2 N$ boundary points whose end points form a planar link pattern $\mathcal{A}^{\delta} \in \mathrm{LP}_{\varsigma}$. We have

$$
\lim _{\delta \rightarrow 0} \mathbb{P}\left[\mathcal{A}^{\delta}=\hat{\alpha}\right]=\mathcal{M}_{\omega, \tau(\hat{\alpha})} \frac{\mathcal{Z}_{\hat{\alpha}}\left(\Omega ; y_{1}, \ldots, y_{2 N}\right)}{\mathcal{Z}_{\mathrm{mGFF}}^{(N)}\left(\Omega ; y_{1}, \ldots, y_{2 N}\right)}, \quad \text { for all } \hat{\alpha} \in \mathrm{LP}_{\varsigma},
$$

where the coefficient $\mathcal{M}_{\omega, \tau(\hat{\alpha})}$ is given by Lemma 5.9, the function $\mathcal{Z}_{\hat{\alpha}}$ is given by Proposition 5.6 and Corollary 5.8, and

$$
\mathcal{Z}_{\mathrm{mGFF}}^{(N)}\left(\Omega ; y_{1}, \ldots, y_{2 N}\right)=\sum_{\hat{\alpha} \in \mathrm{LP}_{\varsigma}} \mathcal{M}_{\omega, \tau(\hat{\alpha})} \mathcal{Z}_{\hat{\alpha}}\left(\Omega ; y_{1}, \ldots, y_{2 N}\right)
$$

The definition for $\mathcal{M}_{\omega, \tau(\hat{\alpha})}$ and $\mathcal{Z}_{\hat{\alpha}}$ is quite involved, and we omit it from the introduction. Nevertheless, let us mention in the introduction nice properties that $\mathcal{Z}_{\hat{\alpha}}$ enjoys. First of all, they are conformally covariant: for any polygon $\left(\Omega ; y_{1}, \ldots, y_{2 N}\right)$ such that $y_{1}, \ldots, y_{2 N}$ lie on sufficiently regular segments of $\partial \Omega$ (e.g. $C^{1+\epsilon}$ for some $\epsilon>0$ ) and any conformal map $\varphi$ on $\Omega$, we have

$$
\mathcal{Z}_{\hat{\alpha}}\left(\Omega ; y_{1}, \ldots, y_{2 N}\right)=\prod_{i=1}^{2 N} \varphi^{\prime}\left(y_{i}\right) \times \mathcal{Z}_{\hat{\alpha}}\left(\varphi(\Omega) ; \varphi\left(y_{1}\right), \ldots, \varphi\left(y_{2 N}\right)\right) .
$$

When $\Omega=\mathbb{H}$ and $y_{1}<\cdots<y_{2 N}$, we write

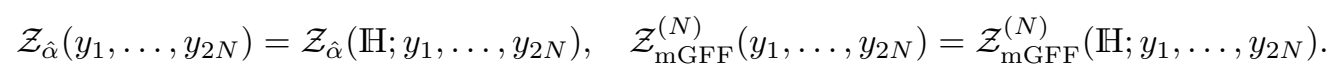

Then, we have

$$
\mathcal{Z}_{\mathrm{mGFF}}^{(N)}\left(y_{1}, \ldots, y_{2 N}\right)=\prod_{1 \leq i<j \leq 2 N}\left(y_{j}-y_{i}\right)^{2(-1)^{j-i}} .
$$

Proposition 1.2. Fix $N \geq 1$ and the index valences $\varsigma=(2, \ldots, 2)$ of length $2 N$. For any $\hat{\alpha} \in \mathrm{LP}_{\varsigma}$, the function $\mathcal{Z}_{\hat{\alpha}}: \mathfrak{X}_{2 N} \rightarrow \mathbb{R}_{>0}$ given by Proposition 5.6 and Corollary 5.8 satisfies the following PDE system: for all $j \in\{1, \ldots, 2 N\}$,

$$
\begin{gathered}
{\left[\frac{\partial^{3}}{\partial y_{j}^{3}}-4 \mathcal{L}_{-2}^{(j)} \frac{\partial}{\partial y_{j}}+2 \mathcal{L}_{-3}^{(j)}\right] \mathcal{Z}_{\hat{\alpha}}\left(y_{1}, \ldots, y_{2 N}\right)=0,} \\
\text { where } \mathcal{L}_{-2}^{(j)}:=\sum_{i \neq j}\left(\frac{1}{\left(y_{i}-y_{j}\right)^{2}}-\frac{1}{y_{i}-y_{j}} \frac{\partial}{\partial y_{i}}\right), \\
\mathcal{L}_{-3}^{(j)}:=\sum_{i \neq j}\left(\frac{2}{\left(y_{i}-y_{j}\right)^{3}}-\frac{1}{\left(y_{i}-y_{j}\right)^{2}} \frac{\partial}{\partial y_{i}}\right) .
\end{gathered}
$$

In Section 2, we will give preliminaries on planar link patterns and SLEs. In Section 3, we will introduce multiple SLE partition functions and prove a preliminary result about "fusion" of partition functions-Proposition 3.1. In Section 4, we will introduce continuum GFF and prove a result on connection probabilities-Theorem 4.1. In Section 5, we will introduce metric graph GFF and complete the proof of Theorem 1.1 and (1.6), (1.7) and (1.8) by combining the results from preceding sections. 


\section{Crossing probabilities in metric graph GFF}

The proof for Theorem 1.1 relies on the following three ingredients: a). Aru-LupuSepúlveda's work on the convergence of first passage sets of metric graph GFF [ALS20], see Sections 5.2 and 5.3. b). A generalization of Peltola and the second author's work [PW19] on crossing probabilities in continuum GFF, see Theorem 4.1. c). Analysis on the asymptotics of multiple $\mathrm{SLE}_{4}$ partition functions, see Proposition 5.6. With these three at hand, let us briefly describe how we derive Theorem 1.1 with $N=2$ which gives (1.3). The proof for general $N$ uses a similar idea. Our strategy is as follows: First, we use a) to show that the frontiers of first passage set of metric graph GFF converge to level lines of continuum GFF with boundary data $(-2 \lambda, 2 \lambda,-2 \lambda, 2 \lambda)$ and proper heights, see Figure 1. Second, we use b) to calculate the crossing probabilities in continuum GFF with boundary data $(-2 \lambda, 0,2 \lambda, 0,-2 \lambda, 0,2 \lambda, 0)$, see Figure 5 . Finally, in Figure 5 , we let $x_{1}, x_{2} \rightarrow y_{1}$, and $x_{3}, x_{4} \rightarrow y_{2}$ and $x_{5}, x_{6} \rightarrow y_{3}$ and $x_{7}, x_{8} \rightarrow y_{4}$, then the four level lines in Figure 5 become the level lines in Figure 1 and, due to $\mathrm{c}$ ), the crossing probabilities calculated in the second step admit limits which give the desired probability in (1.3). See Corollary 5.10.

The proof for Proposition 1.2 relies on Proposition 3.1. Note that the third order PDEs are not surprising. SLE partition functions are solutions to 2nd order PDEs (3.2) and they can be understood as certain correlation functions in terms of conformal field theory (CFT). Then the third order PDEs can be obtained by specific fusion channel, see [BB03, BB04, BBK05, FK04, Dub15, KP16, Pel20, Pel19]. See also discussion after Proposition 3.1. The 2nd order PDEs (3.2) arise from stochastic differentials of certain local martingales and SLE partition functions are related to crossing probabilities for the critical statistical physics models, see [FSKZ17, KKP20, PW19, PW18]. However, there seems no known direct probabilistic interpretation of higher order PDEs of CFT before. In this sense, our work provides an example that gives a probabilistic interpretation to higher order PDEs of CFT.

\section{Preliminaries}

\subsection{Planar pair partitions and Dyck paths}

In this section, we will give a one-to-one correspondence between planar pair partitions and Dyck paths. A Dyck path is a walk on $\mathbb{Z}_{\geq 0}$ with steps of length one, starting and ending at zero: $\alpha:\{0,1, \ldots, 2 N\} \rightarrow \mathbb{Z}_{\geq 0}$ such that $\alpha(0)=\alpha(2 N)=0$, and $|\alpha(k)-\alpha(k-1)|=1$ for all $k \in\{1,2, \ldots, 2 N\}$. For $N \geq 1$, we denote the set of all Dyck paths of $2 N$ steps by $\mathrm{DP}_{\mathrm{N}}$. There is a natural partial order on Dyck paths:

$$
\alpha \preceq \beta \text { if and only if } \alpha(k) \leq \beta(k) \text {, for all } k \in\{0,1, \ldots, 2 N\} \text {. }
$$

We set $\mathrm{DP}=\bigsqcup_{\mathrm{N} \geq 0} \mathrm{DP}_{\mathrm{N}}$.

To each planar pair partition $\alpha \in \mathrm{PP}_{\mathrm{N}}$, we write it as

$$
\begin{gathered}
\alpha=\left\{\left\{a_{1}, b_{1}\right\}, \ldots,\left\{a_{N}, b_{N}\right\}\right\}, \\
\text { where } a_{1}<a_{2}<\cdots<a_{N} \text { and } a_{j}<b_{j}, \text { for all } j \in\{1, \ldots, N\} .
\end{gathered}
$$

We associate it with a Dyck path, also denoted by $\alpha \in \mathrm{DP}_{\mathrm{N}}$, as follows. We set $\alpha(0)=0$ and, for all $k \in\{1, \ldots, 2 N\}$, we set

$$
\alpha(k)= \begin{cases}\alpha(k-1)+1, & \text { if } k \in\left\{a_{1}, a_{2}, \ldots, a_{N}\right\}, \\ \alpha(k-1)-1, & \text { if } k \in\left\{b_{1}, b_{2}, \ldots, b_{N}\right\} .\end{cases}
$$

One may check, this defines a Dyck path $\alpha \in \mathrm{DP}_{\mathrm{N}}$. Conversely, for any Dyck path $\alpha:\{0,1, \ldots, 2 N\} \rightarrow \mathbb{Z}_{\geq 0}$, we associate a planar pair partition $\alpha$ by giving to each up-step 


\section{Crossing probabilities in metric graph GFF}

(i.e., step away from zero) an index $a_{r}$, for $r=1,2, \ldots, N$, and to each down-step (i.e., step towards zero) an index $b_{s}$, for $s=1,2, \ldots, N$, and setting $\alpha:=\left\{\left\{a_{1}, b_{1}\right\}, \ldots,\left\{a_{N}, b_{N}\right\}\right\}$. These two mappings define a bijection between $\mathrm{PP}_{\mathrm{N}}$ and $\mathrm{DP}_{\mathrm{N}}$. We thus identify the elements $\alpha$ of these two sets and use the indistinguishable notation $\alpha \in \mathrm{PP}_{\mathrm{N}}$ and $\alpha \in \mathrm{DP}_{\mathrm{N}}$ for both. See Figure 2 .

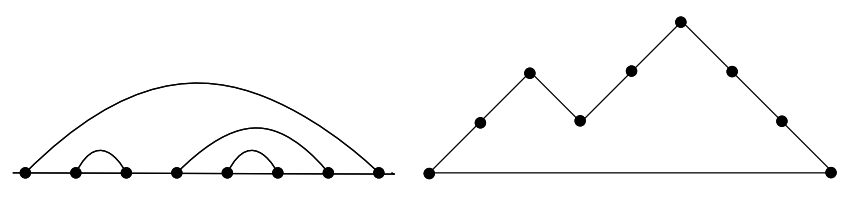

Figure 2: Illustration of the bijection $\mathrm{PP}_{\mathrm{N}} \leftrightarrow \mathrm{DP}_{\mathrm{N}}$, identifying planar pair partition and Dyck path for $\alpha=\{\{1,8\},\{2,3\},\{4,7\},\{5,6\}\}$.

For a Dyck path $\alpha \in \mathrm{DP}_{\mathrm{N}}$, we say that $\alpha$ has a local maximum at $j$ if $\alpha(j)-\alpha(j-1)=1$ and $\alpha(j+1)-\alpha(j)=-1$, and we denote $\wedge^{j} \in \alpha$; we say that $\alpha$ has a local minimum at $j$ if $\alpha(j)-\alpha(j-1)=-1$ and $\alpha(j+1)-\alpha(j)=1$, and we denote $\vee_{j} \in \alpha$; we say that $\alpha$ has a slope at $j$ if otherwise, and we denote $\times_{j} \in \alpha$. We say that $\alpha$ has a local extremum at $j$ if $\alpha$ has a local minimum or maximum at $j$, and we denote $\diamond_{j} \in \alpha$.

If a planar pair partition $\alpha \in \mathrm{PP}_{\mathrm{N}}$ has a link $\{j, j+1\} \in \alpha$, then $\wedge^{j} \in \alpha$. Let $\alpha /\{j, j+1\}$ denote the planar pair partition by removing from $\alpha$ the link $\{j, j+1\}$ and relabelling the remaining indices by $1,2, \ldots, 2 N-2$. In terms of Dyck path, we denote this operation by $\alpha / \wedge^{j} \in \mathrm{DP}_{\mathrm{N}-1}$. We define operation $\alpha / \vee_{j} \in \mathrm{DP}_{\mathrm{N}-1}$ analogously when $\alpha$ has a local minimum at $j$. When $\alpha$ has a local extremum at $j$, we denote such operation by $\alpha / \diamond_{j}$. If $\alpha$ has a local minimum at $j$, we associate $\alpha$ with another Dyck path by converting the local minimum at $j$ to local maximum, and denote this operation by $\alpha \uparrow \diamond_{j}$.

\subsection{From planar link pattern to planar pair partition}

Fix an index valences $\varsigma=\left(s_{1}, \ldots, s_{p}\right) \in \mathbb{Z}_{>0}^{p}$ such that $\sum_{i=1}^{p} s_{i}$ is even and we denote this even number by $2 \ell$. Recall that $\mathrm{LP}_{\varsigma}$ is the collection of all planar $\ell$-link patterns of $p$ points with index valences $\varsigma$. We define a natural map which associates to each planar link pattern a planar pair partition. This map, denoted by

$$
\tau: \mathrm{LP}_{\varsigma} \rightarrow \mathrm{PP}_{\ell}, \quad \hat{\alpha} \mapsto \tau(\hat{\alpha}),
$$

is defined as following: in $\hat{\alpha}$, for each $j \in\{1,2, \ldots, p\}$, we split the $j$ th point to $s_{j}$ distinct points and attach the $s_{j}$ links of $\hat{\alpha}$ ending there to these new $s_{j}$ points so that each of them has valence one. See Figure 3.

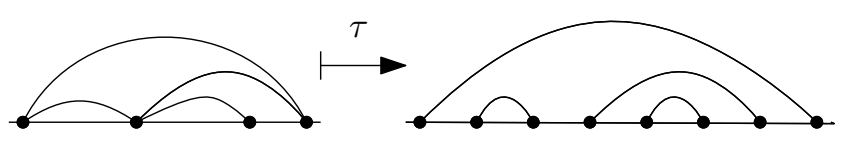

Figure 3: In this figure, we have a planar link pattern with index valences $\varsigma=(2,3,1,2)$. It is associated to a planar pair partition by splitting the four points into eight points according to the valences and attaching the corresponding links.

In this article, we are interested in planar link patterns with index valences $\varsigma=$ $(2, \ldots, 2)$. Fix $N \geq 1$ and the index valences $\varsigma=(2, \ldots, 2)$ of length $2 N$. Then $\tau$ introduces a bijection between $\mathrm{LP}_{\varsigma}$ and the collection $\left\{\beta \in \mathrm{PP}_{2 \mathrm{~N}}: \wedge_{2 \mathrm{j}-1} \notin \beta\right.$, for all $\left.1 \leq \mathrm{j} \leq 2 \mathrm{~N}\right\}$. 


\section{Crossing probabilities in metric graph GFF}

\subsection{Loewner chain and SLE}

We call a compact subset $K$ of $\overline{\mathrm{H}}$ an $\mathbb{H}$-hull if $\mathbb{H} \backslash K$ is simply connected. Riemann's mapping theorem implies that there exists a unique conformal map $g_{K}$ from $\mathbb{H} \backslash K$ onto $\mathbb{H}$ with the property that $\lim _{z \rightarrow \infty}\left|g_{K}(z)-z\right|=0$. We say that $g_{K}$ is normalized at $\infty$.

Consider families of conformal maps $\left(g_{t}, t \geq 0\right)$ obtained by solving the Loewner equation: for each $z \in \mathbb{H}$,

$$
\partial_{t} g_{t}(z)=\frac{2}{g_{t}(z)-W_{t}}, \quad g_{0}(z)=z,
$$

where $\left(W_{t}, t \geq 0\right)$ is a real-valued continuous function, which we call the driving function. Let $T_{z}$ be the swallowing time of $z$ defined as $\sup \left\{t \geq 0: \inf _{s \in[0, t]}\left|g_{s}(z)-W_{s}\right|>0\right\}$. Denote $K_{t}:=\overline{\left\{z \in \mathbb{H}: T_{z} \leq t\right\}}$. Then, $g_{t}$ is the unique conformal map from $H_{t}:=\mathbb{H} \backslash K_{t}$ onto $\mathbb{H}$ normalized at $\infty$. The collection of H-hulls $\left(K_{t}, t \geq 0\right)$ associated with such maps is called a Loewner chain.

Fix $\kappa>0$. The Schramm-Loewner Evolution $\mathrm{SLE}_{\kappa}$ in $\mathbb{H}$ from 0 to $\infty$ is the random Loewner chain $\left(K_{t}, t \geq 0\right)$ driven by $W_{t}=\sqrt{\kappa} B_{t}$, where $\left(B_{t}, t \geq 0\right)$ is the standard one-dimensional Brownian motion. Rohde-Schramm prove in [RS05] that $\left(K_{t}, t \geq 0\right)$ is almost surely generated by a continuous transient curve, i.e., there almost surely exists a continuous curve $\eta$ such that for each $t \geq 0, H_{t}$ is the unbounded connected component of $\mathbb{H} \backslash \eta[0, t]$ and $\lim _{t \rightarrow \infty}|\eta(t)|=\infty$. This random curve is called the SLE $\mathrm{S}_{\kappa}$ trace in $\mathbb{H}$ from 0 to $\infty$. When $\kappa \in(0,4]$, the $\mathrm{SLE}_{\kappa}$ curves are simple; when $\kappa \in(4,8)$, they have self-touchings; when $\kappa \geq 8$, they are space-filling. In this article, we focus on $\kappa=4$ as $\mathrm{SLE}_{4}$ is the level line of Gaussian free field, see Section 4.

\section{Partition functions for multiple SLEs}

At the beginning of this section, we will give a summary on "pure partition functions". As it is more convenient to see the connection to previous works, we write the summary for general $\kappa$. Fix

$$
\kappa \in(0,6], \quad h=\frac{6-\kappa}{2 \kappa}, \quad H=\frac{8-\kappa}{\kappa} .
$$

Pure partition functions for multiple $\mathrm{SLE}_{\kappa}$ is a collection of smooth functions

$$
\mathcal{Z}_{\alpha}: \mathfrak{X}_{2 N} \rightarrow \mathbb{R}
$$

defined on the configuration space $\mathfrak{X}_{2 N}:=\left\{\left(x_{1}, \ldots, x_{2 N}\right) \in \mathbb{R}^{2 N}: x_{1}<\cdots<x_{2 N}\right\}$ and indexed by planar pair partitions $\alpha \in \mathrm{PP}_{\mathrm{N}}$ and satisfying the normalization $\mathcal{Z}_{\emptyset}=1$ and the following properties:

- Partial differential equations of second order (PDE): for all $j \in\{1, \ldots, 2 N\}$,

$$
\left[\frac{\kappa}{2} \frac{\partial^{2}}{\partial x_{j}^{2}}+\sum_{i \neq j}\left(\frac{2}{x_{i}-x_{j}} \frac{\partial}{\partial x_{i}}-\frac{2 h}{\left(x_{i}-x_{j}\right)^{2}}\right)\right] \mathcal{Z}\left(x_{1}, \ldots, x_{2 N}\right)=0
$$

- Möbius covariance (COV): For all Möbius maps $\varphi$ of $\mathbb{H}$ such that $\varphi\left(x_{1}\right)<\cdots<$ $\varphi\left(x_{2 N}\right)$,

$$
\mathcal{Z}\left(x_{1}, \ldots, x_{2 N}\right)=\prod_{i=1}^{2 N} \varphi^{\prime}\left(x_{i}\right)^{h} \times \mathcal{Z}\left(\varphi\left(x_{1}\right), \ldots, \varphi\left(x_{2 N}\right)\right) .
$$




\section{Crossing probabilities in metric graph GFF}

- Asymptotics (ASY): For all $\alpha \in \mathrm{PP}_{\mathrm{N}}$ and for all $j \in\{1, \ldots, 2 N-1\}$ and $\xi \in$ $\left(x_{j-1}, x_{j+2}\right)$, we have

$$
\begin{aligned}
& \lim _{x_{j}, x_{j+1} \rightarrow \xi} \frac{\mathcal{Z}_{\alpha}\left(x_{1}, \ldots, x_{2 N}\right)}{\left(x_{j+1}-x_{j}\right)^{-2 h}} \\
= & \begin{cases}0, & \text { if }\{j, j+1\} \notin \alpha, \\
\mathcal{Z}_{\alpha /\{j, j+1\}}\left(x_{1}, \ldots, x_{j-1}, x_{j+2}, \ldots, x_{2 N}\right), & \text { if }\{j, j+1\} \in \alpha,\end{cases}
\end{aligned}
$$

where $\alpha /\{j, j+1\} \in \mathrm{PP}_{\mathrm{N}-1}$ denotes the link pattern obtained from $\alpha$ by removing the link $\{j, j+1\}$ and relabelling the remaining indices by $1,2, \ldots, 2 N-2$, as defined in Section 2.1.

- Power law bound: For all $\alpha=\left\{\left\{a_{1}, b_{1}\right\}, \ldots,\left\{a_{N}, b_{N}\right\}\right\} \in \mathrm{PP}_{\mathrm{N}}$,

$$
0<\mathcal{Z}_{\alpha}\left(x_{1}, \ldots, x_{2 N}\right) \leq \prod_{i=1}^{N}\left|x_{b_{i}}-x_{a_{i}}\right|^{-2 h}
$$

The uniqueness of such collection of smooth functions was proved in [FK15] ${ }^{1}$ and the existence of such collection was proved in [Wu20] for $\kappa \leq 6$. See [Dub06], [Dub07], [BBK05], [KP16], and [PW19] for earlier works on partition functions.

In (3.4), we see that, if $\{j, j+1\} \in \alpha$, we normalize the function $\mathcal{Z}_{\alpha}$ by $\left(x_{j+1}-x_{j}\right)^{-2 h}$ and we obtain the limiting function $\mathcal{Z}_{\alpha /\{j, j+1\}}$. The goal of this section is to investigate the correct normalization of $\mathcal{Z}_{\alpha}$ when $\{j, j+1\} \notin \alpha$ and to analyze the limiting function.

Proposition 3.1. Fix $\kappa=4$. For $\alpha \in \mathrm{PP}_{\mathrm{N}}$ and for $j \in\{1,2, \ldots, 2 N-1\}$, we assume $\{j, j+1\} \notin \alpha$. For all $\xi \in\left(x_{j-1}, x_{j+2}\right)$, the following limit exists:

$$
\mathcal{Z}_{\alpha / \amalg_{j}}\left(x_{1}, \ldots, x_{j-1}, \xi, x_{j+2}, \ldots, x_{2 N}\right):=\lim _{x_{j}, x_{j+1} \rightarrow \xi} \frac{\mathcal{Z}_{\alpha}\left(x_{1}, \ldots, x_{2 N}\right)}{\left(x_{j+1}-x_{j}\right)^{2 / \kappa}} .
$$

Furthermore, the limiting function $\mathcal{Z}_{\alpha / \amalg_{j}}$ satisfies the following system of $(2 N-1)$ PDEs 2 and the conformal covariance with $\kappa=4$.

- Partial differential equations of second order (PDE): for $n \in\{1, \ldots, 2 N\} \backslash\{j, j+1\}$, we have

$$
\begin{gathered}
{\left[\frac{\partial^{2}}{\partial x_{n}^{2}}-\frac{4}{\kappa} \mathcal{L}_{-2}^{(n)}\right] \mathcal{Z}_{\alpha / \amalg_{j}}\left(x_{1}, \ldots, x_{j}, x_{j+2}, \ldots, x_{2 N}\right)=0} \\
\text { where } \mathcal{L}_{-2}^{(n)}=\sum_{\substack{1 \leq i \leq 2 N, i \neq j, j+1, n}}\left(\frac{h}{\left(x_{i}-x_{n}\right)^{2}}-\frac{1}{x_{i}-x_{n}} \frac{\partial}{\partial x_{i}}\right)+\left(\frac{H}{\left(x_{j}-x_{n}\right)^{2}}-\frac{1}{x_{j}-x_{n}} \frac{\partial}{\partial x_{j}}\right) .
\end{gathered}
$$

\footnotetext{
${ }^{1}$ In fact, [FK15, Lemma 1] proves a much stronger uniqueness, and such stronger uniqueness plays essential role in deriving (3.12). As we will not need this stronger uniqueness directly in the current article, we do not include the precise statement and refer interested readers to [FK15, Lemma 1] and [PW19].

${ }^{2}$ Note that, the operators $\mathcal{L}_{-2}^{(j)}$ and $\mathcal{L}_{-3}^{(j)}$ in Proposition 3.1 are distinct from the ones in Proposition 1.2. In fact, this kind of operators also depends on the index valences of planar link patterns. To simplify notations, we omit the dependence.
} 
- Partial differential equation of third order (PDE):

$$
\begin{gathered}
{\left[\frac{\partial^{3}}{\partial x_{j}^{3}}-\frac{16}{\kappa} \mathcal{L}_{-2}^{(j)} \frac{\partial}{\partial x_{j}}+\frac{8(8-\kappa)}{\kappa^{2}} \mathcal{L}_{-3}^{(j)}\right] \mathcal{Z}_{\alpha / \amalg_{j}}\left(x_{1}, \ldots, x_{j}, x_{j+2}, \ldots, x_{2 N}\right)=0,} \\
\text { where } \mathcal{L}_{-2}^{(j)}=\sum_{\substack{1 \leq i \leq 2 N, i \neq j, j+1}}\left(\frac{h}{\left(x_{i}-x_{j}\right)^{2}}-\frac{1}{\left(x_{i}-x_{j}\right)} \frac{\partial}{\partial x_{i}}\right), \\
\mathcal{L}_{-3}^{(j)}=\sum_{\substack{1 \leq i \leq 2 N, i \neq j, j+1}}\left(\frac{2 h}{\left(x_{i}-x_{j}\right)^{3}}-\frac{1}{\left(x_{i}-x_{j}\right)^{2}} \frac{\partial}{\partial x_{i}}\right) .
\end{gathered}
$$

- Möbius covariance (COV): For all Möbius maps $\varphi$ of $\mathbb{H}$ such that $\varphi\left(x_{1}\right)<\cdots<$ $\varphi\left(x_{2 N}\right)$,

$$
\begin{aligned}
& \mathcal{Z}_{\alpha / \amalg_{j}}\left(x_{1}, \ldots, x_{j}, x_{j+2}, \ldots, x_{2 N}\right) \\
= & \prod_{i} \varphi^{\prime}\left(x_{i}\right)^{\Delta_{i}} \times \mathcal{Z}_{\alpha / \amalg_{j}}\left(\varphi\left(x_{1}\right), \ldots, \varphi\left(x_{j}\right), \varphi\left(x_{j+2}\right), \ldots, \varphi\left(x_{2 N}\right)\right),
\end{aligned}
$$

where $\Delta_{i}=h$ for $i \in\{1, \ldots, j-1, j+2, \ldots, 2 N\}$ and $\Delta_{j}=H$.

The connection of $\mathrm{SLE}_{\kappa}$ with conformal field theory (CFT) is now well-known [BB03, BB04, BBK05, FK04]. In that sense, solutions to PDE (3.2) correspond to correlation functions in CFT with central charge $c=(3 \kappa-8)(6-\kappa) / 2 \kappa$. Then PDE (3.7) and (3.8) come as specific fusion channel of correlation functions in terms of CFT [BBK05, Dub15, KP16]. Note that the parameters $h, H$ in (3.1) coincide with the Kac conformal weights $h_{1,2}$ and $h_{1,3}$. In fact, Peltola proves in [Pel20] a more general conclusion for $\kappa \in(0,8) \backslash \mathbb{Q}$. From there, all conclusions in Proposition 3.1 hold for $\kappa \in(0,8) \backslash \mathbb{Q}$. Our results indicate that a similar conclusion as in [Pel20] also holds for $\kappa=4$. Our method is straight forward but is specific for $\kappa=4$, as our proof uses the explicit formulae for $\mathrm{SLE}_{4}$ partition functions constructed in [PW19]. The explicit formulae involve "conformal block functions" which we will introduce in Section 3.1.

Finally, let us describe the connection between the 2nd order PDE (3.2) and the third order PDE (1.8). Consider $y_{1}<\ldots<y_{2 N}$ and $x_{1}<x_{2}<\cdots<x_{4 N-1}<x_{4 N}$. Fix $\alpha \in \mathrm{PP}_{2 \mathrm{~N}}$ and suppose $\mathcal{Z}_{\alpha}\left(x_{1}, \ldots, x_{4 N}\right)$ satisfies the 2 nd order PDE (3.2). We take limits $x_{2 n-1}, x_{2 n} \rightarrow y_{n}$ for all $n \in\{1,2, \ldots, 2 N\}$ and normalize $\mathcal{Z}_{\alpha}\left(x_{1}, \ldots, x_{4 N}\right)$ properly. From (3.6), we see that the proper normalization should be $\prod_{1 \leq n \leq 2 N}\left(x_{2 n}-x_{2 n-1}\right)^{2 / \kappa}$. We will show in Proposition 5.6 that the function $\mathcal{Z}_{\alpha}\left(x_{1}, x_{2}, \ldots, x_{4 N-1}, x_{4 N}\right)$ normalized by $\prod_{1<n<2 N}\left(x_{2 n}-x_{2 n-1}\right)^{2 / \kappa}$ admits a limit and then show that the limit satisfies the third order PDE (1.8). To check the third order PDE (1.8), we will use PDE (3.8). See Proof of Proposition 1.2 in Section 5.4. Again, our proof is specific for $\kappa=4$. The same conclusion holds for $\kappa \in(0,8) \backslash \mathbb{Q}$ due to [Pel20].

In the rest of the article, we fix $\kappa=4$.

\subsection{Conformal block functions}

For $\alpha=\left\{\left\{a_{1}, b_{1}\right\}, \ldots,\left\{a_{N}, b_{N}\right\}\right\} \in \mathrm{DP}_{\mathrm{N}}$ ordered as in (2.2), we define conformal block function $\mathcal{U}_{\alpha}: \mathfrak{X}_{2 N} \rightarrow \mathbb{R}_{>0}$ as follows:

$$
\mathcal{U}_{\alpha}\left(x_{1}, \ldots, x_{2 N}\right):=\prod_{1 \leq i<j \leq 2 N}\left(x_{j}-x_{i}\right)^{\frac{1}{2} \vartheta_{\alpha}(i, j)},
$$

where $\vartheta_{\alpha}(i, j):= \begin{cases}+1, & \text { if } i, j \in\left\{a_{1}, a_{2}, \ldots, a_{N}\right\}, \text { or } i, j \in\left\{b_{1}, b_{2}, \ldots, b_{N}\right\}, \\ -1, & \text { otherwise. }\end{cases}$ 


\section{Crossing probabilities in metric graph GFF}

The function $\mathcal{U}_{\alpha}$ satisfies the second order PDEs (3.2), see [PW19, Lemma 6.4]. These functions appear in CFT as "conformal blocks". In particular, there are analog of such functions for $\kappa \in(0,8) \backslash \mathbb{Q}$ discussed in [KKP19] in terms of CFT.

Next, we give the relation between the two collections $\left\{\mathcal{Z}_{\alpha}: \alpha \in \mathrm{PP}_{\mathrm{N}}\right\}$ and $\left\{\mathcal{U}_{\alpha}\right.$ : $\left.\alpha \in \mathrm{DP}_{\mathrm{N}}\right\}$ : they are related by a linear transformation. To give the transformation, we introduce a binary relation $\stackrel{()}{\longleftarrow}$. Let $\alpha=\left\{\left\{a_{1}, b_{1}\right\}, \ldots,\left\{a_{N}, b_{N}\right\}\right\} \in \mathrm{PP}_{\mathrm{N}}$ be ordered as in (2.2). Let $\beta \in \mathrm{PP}_{\mathrm{N}}$. Then, $\alpha \stackrel{()}{\longleftarrow} \beta$ if and only if there exists a permutation $\sigma \in \mathfrak{S}_{N}$ such that

$$
\beta=\left\{\left\{a_{1}, b_{\sigma(1)}\right\}, \ldots,\left\{a_{N}, b_{\sigma(N)}\right\}\right\} .
$$

Note that the right-hand side in the above expression may not be ordered as in (2.2). We denote by $\mathcal{M}=\left(\mathcal{M}_{\alpha, \beta}\right)$ the $C_{N} \times C_{N}$ incidence matrix of this relation:

$$
\mathcal{M}_{\alpha, \beta}= \begin{cases}1, & \text { if } \alpha \stackrel{()}{\longleftarrow} \beta \\ 0, & \text { if else. }\end{cases}
$$

We collect some properties of $\mathcal{M}$ in the following lemma. Recall from Section 2.1 that each planar pair partition $\alpha \in \mathrm{PP}_{\mathrm{N}}$ is associated with a Dyck path which we also denote by $\alpha \in \mathrm{DP}_{\mathrm{N}}$.

Lemma 3.2. The matrix $\mathcal{M}$ is invertible and we denote its inverse by $\mathcal{M}^{-1}=\left(\mathcal{M}_{\alpha, \beta}^{-1}\right)$. The entry $\mathcal{M}_{\alpha, \beta}^{-1}$ is non-zero if and only if $\alpha \preceq \beta$ as in (2.1). Furthermore, we have the following properties of $\mathcal{M}^{-1}$. Suppose $\alpha, \beta \in \mathrm{DP}_{\mathrm{N}}$.

- Suppose $\wedge^{j} \notin \alpha$ and $\vee_{j} \in \beta$. Then $\alpha \preceq \beta$ if and only if $\alpha \preceq \beta \uparrow \diamond_{j}$.

- Suppose $\wedge^{j} \notin \alpha, \vee_{j} \in \beta$ and $\alpha \preceq \beta$. Then $\mathcal{M}_{\alpha, \beta}^{-1}=-\mathcal{M}_{\alpha, \beta \uparrow \diamond_{j}}^{-1}$.

Proof. See [PW19, Proposition 2.9 and Lemma 2.10].

Now, we are ready to state the linear transformation between the two collections $\left\{\mathcal{Z}_{\alpha}: \alpha \in \mathrm{PP}_{\mathrm{N}}\right\}$ and $\left\{\mathcal{U}_{\alpha}: \alpha \in \mathrm{DP}_{\mathrm{N}}\right\}:$ (see [PW19, Theorem 1.5])

$$
\left\{\begin{array}{l}
\mathcal{U}_{\alpha}\left(x_{1}, \ldots, x_{2 N}\right)=\sum_{\beta \in \mathrm{PP}_{\mathrm{N}}} \mathcal{M}_{\alpha, \beta} \mathcal{Z}_{\beta}\left(x_{1}, \ldots, x_{2 N}\right) \\
\mathcal{Z}_{\alpha}\left(x_{1}, \ldots, x_{2 N}\right)=\sum_{\beta \in \mathrm{DP}_{\mathrm{N}}} \mathcal{M}_{\alpha, \beta}^{-1} \mathcal{U}_{\beta}\left(x_{1}, \ldots, x_{2 N}\right)
\end{array}\right.
$$

\subsection{Asymptotics of partition functions}

In this section, we will analyze the asymptotics of pure partition functions and conformal block functions as $x_{j}, x_{j+1} \rightarrow \xi$. Note that, we will use the following basic facts about $\vartheta_{\alpha}$ through calculation without notice: for distinct $i, s, t \in\{1,2, \ldots, 2 N\}$, we have

$$
\vartheta_{\alpha}(t, s)^{2}=1, \quad \vartheta_{\alpha}(t, i) \vartheta_{\alpha}(s, i)=\vartheta_{\alpha}(t, s) .
$$

Lemma 3.3. The collection $\left\{\mathcal{U}_{\alpha}: \alpha \in \mathrm{DP}\right\}$ of conformal block functions satisfy the following asymptotic property: for any $j \in\{1, \ldots, 2 N-1\}$ and $x_{1}<x_{2}<\cdots<x_{j-1}<$ $\xi<x_{j+2}<\cdots<x_{2 N}$,

$$
\begin{aligned}
& \lim _{\substack{\tilde{x}_{j}, \tilde{x}_{j+1} \rightarrow \xi, \tilde{x}_{i} \rightarrow x_{i} \text { for } i \neq j, j+1}} \frac{\mathcal{U}_{\alpha}\left(\tilde{x}_{1}, \ldots, \tilde{x}_{2 N}\right)}{\left(\tilde{x}_{j+1}-\tilde{x}_{j}\right)^{-1 / 2}}= \begin{cases}\mathcal{U}_{\alpha / \wedge^{j}}\left(x_{1}, \ldots, x_{j-1}, x_{j+2}, \ldots, x_{2 N}\right), & \text { if } \wedge^{j} \in \alpha, \\
\mathcal{U}_{\alpha / \vee_{j}}\left(x_{1}, \ldots, x_{j-1}, x_{j+2}, \ldots, x_{2 N}\right), & \text { if } \vee_{j} \in \alpha,\end{cases} \\
& \lim _{\substack{\tilde{x}_{j}, \tilde{x}_{j+1} \rightarrow \xi, \tilde{x}_{i} \rightarrow x_{i} \text { for } i \neq j, j+1}} \frac{\mathcal{U}_{\alpha}\left(\tilde{x}_{1}, \ldots, \tilde{x}_{2 N}\right)}{\left(\tilde{x}_{j+1}-\tilde{x}_{j}\right)^{1 / 2}}=\mathcal{U}_{\alpha / \times_{j}}\left(x_{1}, \ldots, x_{j-1}, \xi, x_{j+2}, \ldots, x_{2 N}\right), \quad \text { if } \times_{j} \in \alpha,
\end{aligned}
$$




\section{Crossing probabilities in metric graph GFF}

where

$$
\mathcal{U}_{\alpha / \times_{j}}\left(x_{1}, \ldots, x_{j-1}, \xi, x_{j+2}, \ldots, x_{2 N}\right):=\prod_{\substack{1 \leq t<s \leq 2 N \\ t, s \neq j, j+1}}\left(x_{s}-x_{t}\right)^{\frac{1}{2} \vartheta_{\alpha}(t, s)} \prod_{\substack{1 \leq i \leq 2 N \\ i \neq j, j+1}}\left|x_{i}-\xi\right|^{\vartheta_{\alpha}(i, j)} .
$$

Proof. The asymptotics in (3.13) is proved in [PW19, Lemma 6.6]. It remains to show (3.14). By definition,

$\frac{\mathcal{U}_{\alpha}\left(\tilde{x}_{1}, \ldots, \tilde{x}_{2 N}\right)}{\left(\tilde{x}_{j+1}-\tilde{x}_{j}\right)^{1 / 2}}=\prod_{\substack{1 \leq t<s \leq 2 N \\ t, s \neq j, j+1}}\left(\tilde{x}_{s}-\tilde{x}_{t}\right)^{\frac{1}{2} \vartheta_{\alpha}(t, s)} \prod_{\substack{1 \leq i \leq 2 N \\ i \neq j, j+1}}\left|\tilde{x}_{i}-\tilde{x}_{j}\right|^{\frac{1}{2} \vartheta_{\alpha}(i, j)} \prod_{\substack{1 \leq i \leq 2 N \\ i \neq j, j+1}}\left|\tilde{x}_{i}-\tilde{x}_{j+1}\right|^{\frac{1}{2} \vartheta_{\alpha}(i, j+1)}$.

Since $\times_{j} \in \alpha$, we have $\vartheta_{\alpha}(i, j)=\vartheta_{\alpha}(i, j+1)$. By taking limit, we obtain (3.14).

Lemma 3.4. The collection $\left\{\mathcal{Z}_{\alpha}: \alpha \in \mathrm{PP}\right\}$ of pure partition functions satisfy the following asymptotic property: for any $j \in\{1, \ldots, 2 N-1\}$ and $x_{1}<x_{2}<\cdots<x_{j-1}<\xi<$ $x_{j+2}<\cdots<x_{2 N}$,

$\lim _{\substack{\tilde{x}_{j}, \tilde{x}_{j+1} \rightarrow \xi, \tilde{x}_{i} \rightarrow x_{i} \text { for } i \neq j, j+1}} \frac{\mathcal{Z}_{\alpha}\left(\tilde{x}_{1}, \ldots, \tilde{x}_{2 N}\right)}{\left(\tilde{x}_{j+1}-\tilde{x}_{j}\right)^{-1 / 2}}=\mathcal{Z}_{\alpha / \wedge_{j}}\left(x_{1}, \ldots, x_{j-1}, x_{j+2}, \ldots, x_{2 N}\right), \quad$ if $\{j, j+1\} \in \alpha$,

$\lim _{\substack{\tilde{x}_{j}, \tilde{x}_{j+1} \rightarrow \xi, \tilde{x}_{i} \rightarrow x_{i} \text { for } i \neq j, j+1}} \frac{\mathcal{Z}_{\alpha}\left(\tilde{x}_{1}, \ldots, \tilde{x}_{2 N}\right)}{\left(\tilde{x}_{j+1}-\tilde{x}_{j}\right)^{1 / 2}}=\mathcal{Z}_{\alpha / \amalg_{j}}\left(x_{1}, \ldots, x_{j-1}, \xi, x_{j+2}, \ldots, x_{2 N}\right), \quad$ if $\{j, j+1\} \notin \alpha$,

where

$$
\begin{aligned}
& \mathcal{Z}_{\alpha / \amalg_{j}}:=\sum_{\vee_{j} \in \beta} \mathcal{M}_{\alpha, \beta}^{-1} \mathcal{V}_{\beta / \vee_{j}}+\sum_{\times_{j} \in \beta} \mathcal{M}_{\alpha, \beta}^{-1} \mathcal{U}_{\beta / \times_{j}}, \\
& \mathcal{V}_{\beta / \vee_{j}}\left(x_{1}, \ldots, x_{j-1}, \xi, x_{j+2}, \ldots, x_{2 N}\right):=\prod_{\substack{1 \leq t<s \leq 2 N \\
t, s \neq j, j+1}}\left(x_{s}-x_{t}\right)^{\frac{1}{2} \vartheta_{\beta}(t, s)} \sum_{\substack{1 \leq i \leq 2 N \\
i \neq j, j+1}} \frac{\vartheta_{\beta}(i, j)}{x_{i}-\xi} .
\end{aligned}
$$

Proof. The asymptotics in (3.16) is proved in [PW19, Lemma 6.7]. It remains to show (3.17). In the following, we assume $\{j, j+1\} \notin \alpha$. From Lemma 3.2 and (3.12), we have

$$
\begin{aligned}
& \mathcal{Z}_{\alpha}\left(\tilde{x}_{1}, \ldots, \tilde{x}_{2 N}\right) \\
& =\sum_{\substack{\alpha \_\beta \\
\vee_{j} \in \beta}} \mathcal{M}_{\alpha, \beta}^{-1} \mathcal{U}_{\beta}\left(\tilde{x}_{1}, \ldots, \tilde{x}_{2 N}\right)+\sum_{\substack{\alpha \_\beta \\
\wedge_{j} \in \beta}} \mathcal{M}_{\alpha, \beta}^{-1} \mathcal{U}_{\beta}\left(\tilde{x}_{1}, \ldots, \tilde{x}_{2 N}\right)+\sum_{\substack{\alpha \preceq \beta \\
x_{j} \in \beta}} \mathcal{M}_{\alpha, \beta}^{-1} \mathcal{U}_{\beta}\left(\tilde{x}_{1}, \ldots, \tilde{x}_{2 N}\right) .
\end{aligned}
$$

From Lemma 3.2, for every $\beta \in \mathrm{DP}_{\mathrm{N}}$ with $\vee_{j} \in \beta$, we have $\alpha \preceq \beta$ if and only if $\alpha \preceq \beta \uparrow \diamond_{j}$. In such case, we have further that $\mathcal{M}_{\alpha, \beta}^{-1}=-\mathcal{M}_{\alpha, \beta \uparrow \diamond_{j}}^{-1}$. For the first two sums in the right hand side of (3.19), we have

$$
\begin{aligned}
& \sum_{\substack{\alpha \preceq \beta \\
\vee_{j} \in \beta}} \mathcal{M}_{\alpha, \beta}^{-1} \mathcal{U}_{\beta}\left(\tilde{x}_{1}, \ldots, \tilde{x}_{2 N}\right)+\sum_{\substack{\alpha \preceq \beta \\
\wedge j \in \beta}} \mathcal{M}_{\alpha, \beta}^{-1} \mathcal{U}_{\beta}\left(\tilde{x}_{1}, \ldots, \tilde{x}_{2 N}\right) \\
= & \sum_{\substack{\alpha \preceq \beta \\
\vee_{j} \in \beta}} \mathcal{M}_{\alpha, \beta}^{-1}\left(\mathcal{U}_{\beta}-\mathcal{U}_{\beta \uparrow \diamond_{j}}\right)\left(\tilde{x}_{1}, \ldots, \tilde{x}_{2 N}\right) .
\end{aligned}
$$


Fix $\beta$ such that $\alpha \preceq \beta$ and $\vee_{j} \in \beta$, we have

$$
\begin{aligned}
& \left(\mathcal{U}_{\beta}-\mathcal{U}_{\beta \uparrow \diamond_{j}}\right)\left(\tilde{x}_{1}, \ldots, \tilde{x}_{2 N}\right) \\
= & \left(\tilde{x}_{j+1}-\tilde{x}_{j}\right)^{-\frac{1}{2}} \times\left(\prod_{i \neq j, j+1}\left(\frac{\tilde{x}_{i}-\tilde{x}_{j}}{\tilde{x}_{i}-\tilde{x}_{j+1}}\right)^{\frac{1}{2} \vartheta_{\beta}(i, j)}-\prod_{i \neq j, j+1}\left(\frac{\tilde{x}_{i}-\tilde{x}_{j+1}}{\tilde{x}_{i}-\tilde{x}_{j}}\right)^{\frac{1}{2} \vartheta_{\beta}(i, j)}\right) \\
& \times \prod_{\substack{1 \leq t<s \leq 2 N \\
t, s \neq j, j+1}}\left(\tilde{x}_{s}-\tilde{x}_{t}\right)^{\frac{1}{2} \vartheta_{\beta}(t, s)} .
\end{aligned}
$$

Dividing by $\left(\tilde{x}_{j+1}-\tilde{x}_{j}\right)^{1 / 2}$, we have

$$
\lim _{\substack{\tilde{x}_{j}, \tilde{x}_{j+1} \rightarrow \xi, \tilde{x}_{i} \rightarrow x_{i} \text { for } i \neq j, j+1}} \frac{\left(\mathcal{U}_{\beta}-\mathcal{U}_{\beta \uparrow \diamond_{j}}\right)\left(\tilde{x}_{1}, \ldots, \tilde{x}_{2 N}\right)}{\left(\tilde{x}_{j+1}-\tilde{x}_{j}\right)^{\frac{1}{2}}}=\prod_{\substack{1 \leq t<s \leq 2 N \\ t, s \neq j, j+1}}\left(x_{s}-x_{t}\right)^{\frac{1}{2} \vartheta_{\beta}(t, s)} \sum_{\substack{1 \leq i \leq 2 N \\ i \neq j, j+1}} \frac{\vartheta_{\beta}(i, j)}{x_{i}-\xi} .
$$

For the third sum in the right hand side of (3.19), by (3.14), we have

$$
\lim _{\substack{\tilde{x}_{j}, \tilde{x}_{j+1} \rightarrow \xi, \tilde{x}_{i} \rightarrow x_{i} \text { for } i \neq j, j+1}} \frac{\mathcal{U}_{\beta}\left(\tilde{x}_{1}, \ldots, \tilde{x}_{2 N}\right)}{\left(\tilde{x}_{j+1}-\tilde{x}_{j}\right)^{1 / 2}}=\mathcal{U}_{\beta / \times_{j}}\left(x_{1}, \ldots, x_{j-1}, \xi, x_{j+2}, \ldots, x_{2 N}\right) .
$$

Plugging (3.20) and (3.21) into (3.19), we obtain (3.17).

Note that, we use the notation $\alpha / \amalg_{j}$ in (3.18). It can be understood as a general link pattern. For a planar pair partition $\alpha \in \mathrm{PP}_{\mathrm{N}}$, suppose $\times_{j} \in \alpha$ or $\vee_{j} \in \alpha$, we define $\alpha / \amalg_{j}$ to be the $N$-link pattern of $(2 N-1)$ points with index valences $\varsigma=(1, \ldots, 1,2,1, \ldots, 1)$ obtained from $\alpha$ by merging the points $j$ and $j+1$ and relabelling the remaining $(2 N-1)$ indices so that they are the first $(2 N-1)$ integers.

\subsection{Fusion of partition functions}

In this section, we will show that the functions defined in (3.15) and (3.18) satisfy the system of $(2 N-1)$ PDEs in (3.7) and (3.8), and complete the proof of Proposition 3.1.

Lemma 3.5. The function $\mathcal{U}_{\alpha / \times_{j}}$ defined in (3.15) satisfies the second order PDE (3.7) with $\kappa=4$ for $n \in\{1, \ldots, 2 N\} \backslash\{j, j+1\}$.

Proof. Without loss of generality, we assume $j=1$. Note that $h=1 / 4$ and $H=1$ when $\kappa=4$. The second order PDE (3.7) becomes the following: for $n \in\{3,4, \ldots, 2 N\}$,

$$
\begin{gathered}
{\left[\frac{\partial^{2}}{\partial x_{n}^{2}}-\mathcal{L}_{-2}^{(n)}\right] F\left(x_{1}, x_{3}, \ldots, x_{2 N}\right)=0,} \\
\text { where } \mathcal{L}_{-2}^{(n)}=\sum_{\substack{3 \leq i \leq 2 N \\
i \neq n}}\left(\frac{\frac{1}{4}}{\left(x_{i}-x_{n}\right)^{2}}-\frac{1}{x_{i}-x_{n}} \frac{\partial}{\partial x_{i}}\right)+\left(\frac{1}{\left(x_{1}-x_{n}\right)^{2}}-\frac{1}{x_{1}-x_{n}} \frac{\partial}{\partial x_{1}}\right) .
\end{gathered}
$$

The function in (3.15) with $j=1$ becomes

$$
\mathcal{U}_{\alpha / \times_{1}}\left(x_{1}, x_{3}, \ldots, x_{2 N}\right):=\prod_{3 \leq t<s \leq 2 N}\left(x_{s}-x_{t}\right)^{\frac{1}{2} \vartheta_{\alpha}(t, s)} \prod_{3 \leq i \leq 2 N}\left(x_{i}-x_{1}\right)^{\vartheta_{\alpha}(i, 1)} .
$$

It suffices to show that the function in (3.23) solves the second order PDE (3.22).

We write $\boldsymbol{x}=\left(x_{1}, x_{3}, \ldots, x_{2 N}\right)$. We have, for $i \in\{3,4, \ldots, 2 N\}$,

$$
\frac{\frac{\partial}{\partial x_{i}} \mathcal{U}_{\alpha / \times_{1}}(\boldsymbol{x})}{\mathcal{U}_{\alpha / \times_{1}}(\boldsymbol{x})}=\sum_{\substack{3 \leq s \leq 2 N \\ s \neq i}} \frac{\frac{1}{2} \vartheta_{\alpha}(s, i)}{x_{i}-x_{s}}+\frac{\vartheta_{\alpha}(i, 1)}{x_{i}-x_{1}} ; \quad \frac{\frac{\partial}{\partial x_{1}} \mathcal{U}_{\alpha / \times_{1}}(\boldsymbol{x})}{\mathcal{U}_{\alpha / \times_{1}}(\boldsymbol{x})}=\sum_{3 \leq s \leq 2 N} \frac{-\vartheta_{\alpha}(s, 1)}{x_{s}-x_{1}} .
$$


Then, we have

$$
\begin{aligned}
\frac{\mathcal{L}_{-2}^{(n)} \mathcal{U}_{\alpha / \times_{1}}(\boldsymbol{x})}{\mathcal{U}_{\alpha / \times_{1}}(\boldsymbol{x})}= & \sum_{\substack{3 \leq i \leq 2 N, i \neq n}} \frac{\frac{1}{4}-\frac{1}{2} \vartheta_{\alpha}(n, i)}{\left(x_{n}-x_{i}\right)^{2}}+\frac{1-\vartheta_{\alpha}(n, 1)}{\left(x_{n}-x_{1}\right)^{2}} \\
& +\sum_{\substack{3 \leq t<s \leq 2 N, t, s \neq n}} \frac{\frac{1}{2} \vartheta_{\alpha}(t, s)}{\left(x_{n}-x_{t}\right)\left(x_{n}-x_{s}\right)}+\sum_{\substack{3 \leq i \leq 2 N, i \neq n}} \frac{\vartheta_{\alpha}(i, 1)}{\left(x_{n}-x_{i}\right)\left(x_{n}-x_{1}\right)} ; \\
\frac{\frac{\partial^{2}}{\partial x_{n}^{2}} \mathcal{U}_{\alpha / \times_{1}}(\boldsymbol{x})}{\mathcal{U}_{\alpha / \times_{1}}(\boldsymbol{x})}= & \sum_{\substack{3 \leq i \leq 2 N, i \neq n}} \frac{\frac{1}{4}-\frac{1}{2} \vartheta_{\alpha}(n, i)}{\left(x_{n}-x_{i}\right)^{2}}+\frac{1-\vartheta_{\alpha}(n, 1)}{\left(x_{n}-x_{1}\right)^{2}} \\
& +\sum_{\substack{3 \leq t<s \leq 2 N, t, s \neq n}} \frac{\frac{1}{2} \vartheta_{\alpha}(s, t)}{\left(x_{n}-x_{t}\right)\left(x_{n}-x_{s}\right)}+\sum_{\substack{3 \leq i \leq 2 N \\
i \neq n}} \frac{\vartheta_{\alpha}(i, 1)}{\left(x_{n}-x_{i}\right)\left(x_{n}-x_{1}\right)} .
\end{aligned}
$$

These give $\left[\frac{\partial^{2}}{\partial x_{n}^{2}}-\mathcal{L}_{-2}^{(n)}\right] \mathcal{U}_{\alpha / \times_{1}}=0$ as desired.

Lemma 3.6. The function $\mathcal{U}_{\alpha / \times_{j}}$ defined in (3.15) satisfies the third order PDE (3.8) with $\kappa=4$.

Proof. Without loss of generality, we assume $j=1$. The third order PDE (3.8) becomes the following:

$$
\begin{aligned}
& {\left[\frac{\partial^{3}}{\partial x_{1}^{3}}-4 \mathcal{L}_{-2}^{(1)} \frac{\partial}{\partial x_{1}}+2 \mathcal{L}_{-3}^{(1)}\right] F\left(x_{1}, x_{3}, \ldots, x_{2 N}\right)=0,} \\
& \text { where } \mathcal{L}_{-2}^{(1)}=\sum_{3 \leq i \leq 2 N}\left(\frac{\frac{1}{4}}{\left(x_{i}-x_{1}\right)^{2}}-\frac{1}{\left(x_{i}-x_{1}\right)} \frac{\partial}{\partial x_{i}}\right) \\
& \mathcal{L}_{-3}^{(1)}=\sum_{3 \leq i \leq 2 N}\left(\frac{\frac{1}{2}}{\left(x_{i}-x_{1}\right)^{3}}-\frac{1}{\left(x_{i}-x_{1}\right)^{2}} \frac{\partial}{\partial x_{i}}\right)
\end{aligned}
$$

It suffices to show that the function in (3.23) solves the third order PDE (3.24). We write $\boldsymbol{x}=\left(x_{1}, x_{3}, \ldots, x_{2 N}\right)$. We have

$$
\begin{aligned}
& \frac{2 \mathcal{L}_{-3}^{(1)} \mathcal{U}_{\alpha / \times_{1}}(\boldsymbol{x})}{\mathcal{U}_{\alpha / \times_{1}}(\boldsymbol{x})} \\
= & \sum_{3 \leq i \leq 2 N} \frac{1-2 \vartheta_{\alpha}(i, 1)}{\left(x_{i}-x_{1}\right)^{3}}+\sum_{3 \leq t<s \leq 2 N} \frac{\vartheta_{\alpha}(s, t)\left(x_{t}-x_{1}+x_{s}-x_{1}\right)}{\left(x_{t}-x_{1}\right)^{2}\left(x_{s}-x_{1}\right)^{2}} ; \\
& \frac{4 \mathcal{L}_{-2}^{(1)} \frac{\partial}{\partial x_{1}} \mathcal{U}_{\alpha / \times_{1}}(\boldsymbol{x})}{\mathcal{U}_{\alpha / \times_{1}}(\boldsymbol{x})} \\
= & \sum_{3 \leq i \leq 2 N} \frac{4-5 \vartheta_{\alpha}(i, 1)}{\left(x_{i}-x_{1}\right)^{3}} \\
& +\sum_{3 \leq t<s \leq 2 N} \frac{4 \vartheta_{\alpha}(s, t)\left(x_{t}-x_{1}+x_{s}-x_{1}\right)-3 \vartheta_{\alpha}(t, 1)\left(x_{t}-x_{1}\right)-3 \vartheta_{\alpha}(s, 1)\left(x_{s}-x_{1}\right)}{\left(x_{t}-x_{1}\right)^{2}\left(x_{s}-x_{1}\right)^{2}} \\
& +\sum_{3 \leq t<s<n \leq 2 N} \frac{(-6) \vartheta_{\alpha}(t, 1) \vartheta_{\alpha}(s, 1) \vartheta_{\alpha}(n, 1)}{\left(x_{t}-x_{1}\right)\left(x_{s}-x_{1}\right)\left(x_{n}-x_{1}\right)}
\end{aligned}
$$




\section{Crossing probabilities in metric graph GFF}

$$
\begin{aligned}
& \frac{\frac{\partial^{3}}{\partial x_{1}^{3}} \mathcal{U}_{\alpha / \times_{1}}(\boldsymbol{x})}{\mathcal{U}_{\alpha / \times_{1}}(\boldsymbol{x})} \\
= & \sum_{3 \leq i \leq 2 N} \frac{3-3 \vartheta_{\alpha}(i, 1)}{\left(x_{i}-x_{1}\right)^{3}} \\
& +\sum_{3 \leq t<s \leq 2 N} \frac{3\left(\vartheta_{\alpha}(s, t)-\vartheta_{\alpha}(t, 1)\right)\left(x_{t}-x_{1}\right)+3\left(\vartheta_{\alpha}(s, t)-\vartheta_{\alpha}(s, 1)\right)\left(x_{s}-x_{1}\right)}{\left(x_{t}-x_{1}\right)^{2}\left(x_{s}-x_{1}\right)^{2}} \\
& +\sum_{3 \leq t<s<n \leq 2 N} \frac{(-6) \vartheta_{\alpha}(t, 1) \vartheta_{\alpha}(s, 1) \vartheta_{\alpha}(n, 1)}{\left(x_{t}-x_{1}\right)\left(x_{s}-x_{1}\right)\left(x_{n}-x_{1}\right)} .
\end{aligned}
$$

These give $\left[\frac{\partial^{3}}{\partial x_{1}^{3}}-4 \mathcal{L}_{-2}^{(1)} \frac{\partial}{\partial x_{1}}+2 \mathcal{L}_{-3}^{(1)}\right] \mathcal{U}_{\alpha / \times_{1}}=0$ as desired.

Lemma 3.7. The function $\mathcal{Z}_{\alpha / \amalg_{j}}$ defined in (3.18) satisfies the second order PDE (3.7) with $\kappa=4$ for $n \in\{1, \ldots, 2 N\} \backslash\{j, j+1\}$.

Proof. Without loss of generality, we assume $j=1$. The function in (3.18) with $j=1$ becomes $\mathcal{Z}_{\alpha / \amalg_{1}}=\sum_{\beta: \vee_{1} \in \beta} \mathcal{M}_{\alpha, \beta}^{-1} \mathcal{V}_{\beta / \vee_{1}}+\sum_{\beta: \times_{1} \in \beta} \mathcal{M}_{\alpha, \beta}^{-1} \mathcal{U}_{\beta / \times_{1}}$ where

$$
\mathcal{V}_{\beta / \vee_{1}}\left(x_{1}, x_{3}, \ldots, x_{2 N}\right):=\prod_{3 \leq t<s \leq 2 N}\left(x_{s}-x_{t}\right)^{\frac{1}{2} \vartheta_{\beta}(t, s)} \sum_{3 \leq i \leq 2 N} \frac{\vartheta_{\beta}(i, 1)}{x_{i}-x_{1}}
$$

We will show that $\mathcal{Z}_{\alpha / \amalg_{1}}$ satisfies the second order PDE (3.22). From Lemma 3.5, the function $\mathcal{U}_{\beta / \times_{1}}$ satisfies PDE (3.22). It suffcies to show that the function $\mathcal{V}_{\beta / \vee_{1}}$ in (3.25) satisfies PDE (3.22).

We write $\boldsymbol{x}=\left(x_{1}, x_{3}, \ldots, x_{2 N}\right)$ and set

$$
\sigma(\boldsymbol{x})=\sum_{3 \leq i \leq 2 N} \frac{\vartheta_{\beta}(i, 1)}{x_{i}-x_{1}} .
$$

We have, for $i \in\{3,4, \ldots, 2 N\}$,

$$
\begin{aligned}
& \frac{\frac{\partial}{\partial x_{i}} \mathcal{V}_{\beta / \vee_{1}}(\boldsymbol{x})}{\mathcal{V}_{\beta / \vee_{1}}(\boldsymbol{x})}=\sum_{\substack{3 \leq s \leq 2 N \\
s \neq i}} \frac{\frac{1}{2} \vartheta_{\beta}(i, s)}{x_{i}-x_{s}}+\frac{-\vartheta_{\beta}(i, 1)}{\sigma(\boldsymbol{x})\left(x_{i}-x_{1}\right)^{2}}, \\
& \frac{\frac{\partial}{\partial x_{1}} \mathcal{V}_{\beta / \vee_{1}}(\boldsymbol{x})}{\mathcal{V}_{\beta / \vee_{1}}(\boldsymbol{x})}=\sum_{3 \leq s \leq 2 N} \frac{\vartheta_{\beta}(s, 1)}{\sigma(\boldsymbol{x})\left(x_{s}-x_{1}\right)^{2}} .
\end{aligned}
$$

Then we obtain

$$
\begin{aligned}
& \frac{\mathcal{L}_{-2}^{(n)} \mathcal{V}_{\beta / \vee_{1}}(\boldsymbol{x})}{\mathcal{V}_{\beta / \mathbb{V}_{1}}(\boldsymbol{x})} \\
= & \sum_{\substack{3 \leq i \leq 2 N \\
i \neq n}} \frac{\frac{1}{4}-\frac{1}{2} \vartheta_{\beta}(i, n)}{\left(x_{n}-x_{i}\right)^{2}}+\frac{1}{\left(x_{n}-x_{1}\right)^{2}}+\frac{\vartheta_{\beta}(n, 1)}{\sigma(\boldsymbol{x})\left(x_{n}-x_{1}\right)^{3}} \\
& +\sum_{\substack{3 \leq t<s \leq 2 N \\
t, s \neq n}} \frac{\frac{1}{2} \vartheta_{\beta}(t, s)}{\left(x_{n}-x_{s}\right)\left(x_{n}-x_{t}\right)}+\sum_{\substack{3 \leq i \leq 2 N \\
i \neq n}} \frac{-\vartheta_{\beta}(i, 1)}{\sigma(\boldsymbol{x})\left(x_{n}-x_{i}\right)\left(x_{i}-x_{1}\right)\left(x_{n}-x_{1}\right)} ;
\end{aligned}
$$




$$
\begin{aligned}
& \frac{\frac{\partial^{2}}{\partial x_{n}^{2}} \mathcal{V}_{\beta / \vee_{1}}(\boldsymbol{x})}{\mathcal{V}_{\beta / \vee_{1}}(\boldsymbol{x})} \\
= & \sum_{\substack{3 \leq i \leq 2 N, i \neq n}} \frac{\frac{1}{4}-\frac{1}{2} \vartheta_{\beta}(i, n)}{\left(x_{n}-x_{i}\right)^{2}}+\frac{2 \vartheta_{\beta}(n, 1)}{\sigma(\boldsymbol{x})\left(x_{n}-x_{1}\right)^{3}} \\
& +\sum_{\substack{3 \leq t<s \leq 2 N \\
t, s \neq n}} \frac{\frac{1}{2} \vartheta_{\beta}(t, s)}{\left(x_{n}-x_{s}\right)\left(x_{n}-x_{t}\right)}+\sum_{\substack{3 \leq i \leq 2 N \\
i \neq n}} \frac{-\vartheta_{\beta}(i, 1)}{\sigma(\boldsymbol{x})\left(x_{n}-x_{i}\right)\left(x_{n}-x_{1}\right)^{2}} .
\end{aligned}
$$

Taking the difference, we have

$$
\begin{aligned}
& \frac{\frac{\partial^{2}}{\partial x_{n}^{2}} \mathcal{V}_{\beta / \vee_{1}}(\boldsymbol{x})}{\mathcal{V}_{\beta / \vee_{1}}(\boldsymbol{x})}-\frac{\mathcal{L}_{-2}^{(n)} \mathcal{V}_{\beta / \vee_{1}}(\boldsymbol{x})}{\mathcal{V}_{\beta / \vee_{1}}(\boldsymbol{x})} \\
= & \frac{-1}{\left(x_{n}-x_{1}\right)^{2}}+\frac{\vartheta_{\beta}(n, 1)}{\sigma(\boldsymbol{x})\left(x_{n}-x_{1}\right)^{3}}+\sum_{\substack{3 \leq i \leq 2 N \\
i \neq n}} \frac{-\vartheta_{\beta}(i, 1)}{\sigma(\boldsymbol{x})\left(x_{n}-x_{i}\right)\left(x_{n}-x_{1}\right)^{2}} \\
& +\sum_{\substack{3 \leq i \leq 2 N \\
i \neq n}} \frac{\vartheta_{\beta}(i, 1)}{\sigma(\boldsymbol{x})\left(x_{n}-x_{i}\right)\left(x_{i}-x_{1}\right)\left(x_{n}-x_{1}\right)} \\
= & \frac{-1}{\left(x_{n}-x_{1}\right)^{2}}+\frac{\vartheta_{\beta}(n, 1)}{\sigma(\boldsymbol{x})\left(x_{n}-x_{1}\right)^{3}}+\sum_{\substack{3 \leq i \leq 2 N \\
i \neq n}} \frac{\vartheta_{\beta}(i, 1)}{\sigma(\boldsymbol{x})\left(x_{i}-x_{1}\right)\left(x_{n}-x_{1}\right)^{2}} \\
= & \frac{-1}{\left(x_{n}-x_{1}\right)^{2}}+\frac{\vartheta_{\beta}(n, 1)}{\sigma(\boldsymbol{x})\left(x_{n}-x_{1}\right)^{3}}+\frac{\sigma(\boldsymbol{x})-\frac{\vartheta_{\beta}(n, 1)}{x_{n}-x_{1}}}{\sigma(\boldsymbol{x})\left(x_{n}-x_{1}\right)^{2}}=0 .
\end{aligned}
$$

This completes the proof.

Lemma 3.8. The function $\mathcal{Z}_{\alpha / \amalg_{j}}$ defined in (3.18) satisfies the third order PDE (3.8) with $\kappa=4$.

Proof. Without loss of generality, we assume $j=1$. From Lemma 3.6, the function $\mathcal{U}_{\alpha / \times_{1}}$ satisfies PDE (3.24). It suffices to show that the function $\mathcal{V}_{\beta / v_{1}}$ in (3.25) satisfies the third order PDE (3.24). We write $\boldsymbol{x}=\left(x_{1}, x_{3}, \ldots, x_{2 N}\right)$ and set $\sigma(\boldsymbol{x})$ as in (3.26). Then we have

$$
\begin{aligned}
\frac{2 \mathcal{L}_{-3}^{(1)} \mathcal{V}_{\beta / \vee_{1}}(\boldsymbol{x})}{\mathcal{V}_{\beta / \vee_{1}}(\boldsymbol{x})}= & \sum_{3 \leq i \leq 2 N} \frac{1}{\left(x_{i}-x_{1}\right)^{3}}+\sum_{3 \leq i \leq 2 N} \frac{2 \vartheta_{\beta}(i, 1)}{\sigma(\boldsymbol{x})\left(x_{i}-x_{1}\right)^{4}} \\
& +\sum_{3 \leq t<s \leq 2 N} \frac{\vartheta_{\beta}(s, t)\left(x_{t}-x_{1}+x_{s}-x_{1}\right)}{\left(x_{t}-x_{1}\right)^{2}\left(x_{s}-x_{1}\right)^{2}} ; \\
\frac{4 \mathcal{L}_{-2}^{(1)} \frac{\partial}{\partial x_{1}} \mathcal{V}_{\beta / \vee_{1}}(\boldsymbol{x})}{\mathcal{V}_{\beta / \vee_{1}}(\boldsymbol{x})}= & \sum_{3 \leq i \leq 2 N} \frac{8 \vartheta_{\beta}(i, 1)}{\sigma(\boldsymbol{x})\left(x_{i}-x_{1}\right)^{4}}+\sigma(\boldsymbol{x}) \frac{\partial \sigma(\boldsymbol{x})}{\partial x_{1}} ; \\
\frac{\frac{\partial^{3}}{\partial x_{1}^{3}} \mathcal{V}_{\beta / \vee_{1}}(\boldsymbol{x})}{\mathcal{V}_{\beta / \vee_{1}}(\boldsymbol{x})}= & \sum_{3 \leq i \leq 2 N} \frac{6 \vartheta_{\beta}(i, 1)}{\sigma(\boldsymbol{x})\left(x_{i}-x_{1}\right)^{4}} .
\end{aligned}
$$

Therefore,

$$
\frac{\frac{\partial^{3}}{\partial x_{1}^{3}} \mathcal{V}_{\beta / \vee_{1}}(\boldsymbol{x})}{\mathcal{V}_{\beta / \vee_{1}}(\boldsymbol{x})}-\frac{4 \mathcal{L}_{-2}^{(1)} \frac{\partial}{\partial x_{1}} \mathcal{V}_{\beta / \vee_{1}}(\boldsymbol{x})}{\mathcal{V}_{\beta / \vee_{1}}(\boldsymbol{x})}+\frac{2 \mathcal{L}_{-3}^{(1)} \mathcal{V}_{\beta / \vee_{1}}(\boldsymbol{x})}{\mathcal{V}_{\beta / \mathrm{V}_{1}}(\boldsymbol{x})}
$$




$$
\begin{aligned}
& =\sum_{3 \leq i \leq 2 N} \frac{1}{\left(x_{i}-x_{1}\right)^{3}}+\sum_{3 \leq t<s \leq 2 N} \frac{\vartheta_{\beta}(s, t)\left(x_{t}-x_{1}+x_{s}-x_{1}\right)}{\left(x_{t}-x_{1}\right)^{2}\left(x_{s}-x_{1}\right)^{2}}-\sigma(\boldsymbol{x}) \frac{\partial \sigma(\boldsymbol{x})}{\partial x_{1}} \\
& =\left(\sum_{3 \leq t \leq 2 N} \frac{\vartheta_{\beta}(t, 1)}{x_{t}-x_{1}}\right)\left(\sum_{3 \leq s \leq 2 N} \frac{\vartheta_{\beta}(s, 1)}{\left(x_{s}-x_{1}\right)^{2}}\right)-\sigma(\boldsymbol{x}) \frac{\partial \sigma(\boldsymbol{x})}{\partial x_{1}}=0 .
\end{aligned}
$$

This completes the proof.

Proof of Proposition 3.1. The existence of the limit (3.6) is a consequence of (3.17). The limiting function satisfies the PDE system due to Lemmas 3.7 and 3.8. COV (3.9) is a consequence of $\mathrm{COV}$ (3.3) and the existence of the limit (3.6).

\section{Connection probabilities for level lines in GFF}

In this section, we first introduce continuum GFF and level lines in Section 4.1. Then we state the main conclusion of the section-Theorem 4.1-in Section 4.2. This theorem gives the connection probabilities for level lines of GFF in polygons with boundary data given by Dyck paths. The proof of Theorem 4.1 involves several technical lemmas which we find not instructive to include in the main text. We put the proof of these technical lemmas to Appendix A.

\subsection{Continuum GFF and level lines}

In this section, we introduce the Gaussian free field and its level lines. We refer to the literature [She07, SS13, MS16, WW17] for details. Let $\Omega \subsetneq \mathbb{C}$ be a non-empty domain. We denote by $H_{s}(\Omega)$ the space of real-valued smooth functions which are compactly supported in $\Omega$. We equip the space with Dirichlet inner product

$$
(f, g)_{\nabla}:=\frac{1}{2 \pi} \int_{\Omega} \nabla f(z) \cdot \nabla g(z) d^{2} z .
$$

We denote by $H(\Omega)$ the Hilbert space completion of $H_{s}(\Omega)$ with respect to the Dirichlet inner product. A (zero-boundary) Gaussian free field (GFF) $\Gamma$ is an $H(\Omega)$-indexed linear space of random variables, denoted by $(\Gamma, f)_{\nabla}$ for each $f \in H(\Omega)$, such that the map $f \mapsto(\Gamma, f)_{\nabla}$ is linear and each $(\Gamma, f)_{\nabla}$ is a centered Gaussian with variance $(f, f)_{\nabla}$. In general, for any harmonic function $u$ on $\Omega$, we define the GFF with boundary data $u$ by $\Gamma+u$ where $\Gamma$ is the zero-boundary GFF on $\Omega$.

Next, we introduce SLE with force points. We set

$$
\underline{y}^{L}=\left(y^{L, l}<\cdots<y^{L, 1} \leq 0\right) \text { and } \underline{y}^{R}=\left(0 \leq y^{R, 1}<\cdots<y^{R, r}\right),
$$

and

$$
\underline{\rho}^{L}=\left(\rho^{L, l}, \cdots, \rho^{L, 1}\right) \text { and } \underline{\rho}^{R}=\left(\rho^{R, 1}, \cdots, \rho^{R, r}\right),
$$

where $\rho^{q, i} \in \mathbb{R}$, for $q \in\{L, R\}$ and $i \in \mathbb{Z}_{>0}$. An $\operatorname{SLE}_{\kappa}\left(\underline{\rho}^{\mathrm{L}} ; \underline{\rho}^{\mathrm{R}}\right)$ process with force points $\left(\underline{y}^{L} ; \underline{y}^{R}\right)$ is the Loewner evolution driven by $W_{t}$ that solves the following system of integrated SDEs:

$$
\begin{aligned}
W_{t} & =\sqrt{\kappa} B_{t}+\int_{0}^{t} \frac{\rho^{L, i} d s}{W_{s}-V_{s}^{L, i}}+\sum_{i=1}^{r} \int_{0}^{t} \frac{\rho^{R, i} d s}{W_{s}-V_{s}^{R, i}}, \\
V_{t}^{q, i} & =y^{q, i}+\int_{0}^{t} \frac{2 d s}{V_{s}^{q, i}-W_{s}}, \quad \text { for } q \in\{L, R\} \text { and } i \in \mathbb{Z}_{>0},
\end{aligned}
$$




\section{Crossing probabilities in metric graph GFF}

where $B_{t}$ is the one-dimensional Brownian motion. Note that the process $V_{t}^{q, i}$ is the evolution of the point $y^{q, i}$, and we may write $g_{t}\left(y^{q, i}\right)$ for $V_{t}^{q, i}$. We define the continuation threshold of the $\operatorname{SLE}_{\kappa}\left(\underline{\rho}^{\mathrm{L}} ; \underline{\rho}^{\mathrm{R}}\right)$ to be the infimum of the time $t$ for which

$$
\text { either } \sum_{i: V_{t}^{L, i}=W_{t}} \rho^{L, i} \leq-2, \quad \text { or } \sum_{i: V_{t}^{R, i}=W_{t}} \rho^{R, i} \leq-2 .
$$

By [MS16], the $\operatorname{SLE}_{\kappa}\left(\rho^{\mathrm{L}} ; \underline{\rho}^{\mathrm{R}}\right)$ process is well-defined up to the continuation threshold, and it is almost surely generated by a continuous curve up to and including the continuation threshold.

Now, we are ready to introduce level lines of GFF. Let $K=\left(K_{t}, t \geq 0\right)$ be an $\operatorname{SLE}_{4}\left(\rho^{\mathrm{L}} ; \rho^{\mathrm{R}}\right)$ process with force points $\left(y^{L} ; y^{R}\right)$, with $W, V^{q, i}$ solving the SDE system (4.1) with $\kappa=4$. Let $\left(g_{t}, t \geq 0\right)$ be the corresponding family of conformal maps and set $f_{t}:=g_{t}-W_{t}$. Let $u_{t}^{0}$ be the harmonic function on $\mathbb{H}$ with boundary data

$$
\begin{cases}-\lambda\left(1+\sum_{i=0}^{j} \rho^{L, i}\right), & \text { if } x \in\left(f_{t}\left(y^{L, j+1}\right), f_{t}\left(y^{L, j}\right)\right), \\ +\lambda\left(1+\sum_{i=0}^{j} \rho^{R, i}\right), & \text { if } x \in\left(f_{t}\left(y^{R, j}\right), f_{t}\left(y^{R, j+1}\right)\right),\end{cases}
$$

where $\lambda=\pi / 2$ and $\rho^{L, 0}=\rho^{R, 0}=0, y^{L, 0}=0^{-}, y^{L, l+1}=-\infty, y^{R, 0}=0^{+}$, and $y^{R, r+1}=\infty$ by convention. Define $u_{t}(z):=u_{t}^{0}\left(f_{t}(z)\right)$. By [Dub09, SS13], there exists a coupling $(\Gamma, K)$, where $\Gamma$ is a zero-boundary GFF on $\mathbb{H}$, such that the following is true. Let $\tau$ be any $\eta$-stopping time before the continuation threshold. Then, the conditional law of $\Gamma+u_{0}$ restricted to $\mathbb{H} \backslash K_{\tau}$ given $K_{\tau}$ is the same as the law of $\Gamma^{\prime} \circ f_{\tau}+u_{\tau}$ where $\Gamma^{\prime}$ is a zero-boundary GFF. Furthermore, in this coupling, the process $K$ is almost surely determined by $\Gamma$. We refer to the $\operatorname{SLE}_{4}\left(\rho^{\mathrm{L}} ; \rho^{\mathrm{R}}\right)$ in this coupling as the level line of the field $\Gamma+u_{0}$. In general, for $a \in \mathbb{R}$, the level line of $\Gamma+u_{0}$ with height $a$ is the level line of $\Gamma+u_{0}-a$.

\subsection{Connection probabilities}

For $\alpha \in \mathrm{PP}_{\mathrm{N}}$, recall from Section 2.1 that $\alpha$ also denotes the corresponding Dyck path in $\mathrm{DP}_{\mathrm{N}}$. Let $u_{\alpha}$ be the harmonic function on $\mathbb{H}$ with the following boundary data: $\left(x_{0}=-\infty\right.$ and $x_{2 N+1}=\infty$ by convention)

$$
2 \lambda(\alpha(k)-1) \text { on }\left(x_{k}, x_{k+1}\right), \quad \text { for all } k \in\{0,1,2, \ldots, N\} .
$$

With such choice, we see that $u_{\alpha}$ has boundary data $-2 \lambda$ on $\left(-\infty, x_{1}\right) \cup\left(x_{2 N}, \infty\right)$, and has boundary data 0 on $\left(x_{1}, x_{2}\right) \cup\left(x_{2 N-1}, x_{2 N}\right)$. Define

$$
\mathcal{H}_{\alpha}(k)=\lambda(\alpha(k-1)+\alpha(k)-2), \quad \text { for all } k \in\{1,, 2 \ldots, 2 N\} .
$$

We write $\alpha=\left\{\left\{a_{1}, b_{1}\right\}, \ldots,\left\{a_{N}, b_{N}\right\}\right\}$ as ordered in (2.2). Suppose $\Gamma$ is zero-boundary GFF on $\mathbb{H}$, and consider level lines of $\Gamma+u_{\alpha}$. Let $\eta_{a_{i}}$ be the level line of $\Gamma+u_{\alpha}$ starting from $x_{a_{i}}$ with height $\mathcal{H}_{\alpha}\left(a_{i}\right)$. With such choice, the boundary data to the left side of $\eta_{a_{i}}$ is $2 \lambda(\alpha(k-1)-1)$ and the boundary data to the right side of $\eta_{a_{i}}$ is $2 \lambda(\alpha(k)-1)$. Then the $N$ curves $\left\{\eta_{a_{1}}, \eta_{a_{2}}, \ldots, \eta_{a_{N}}\right\}$ are non-intersecting simple curves and their end points form a planar pair partition of the $2 N$ boundary points. We denote this planar pair partition by $\mathcal{A}=\mathcal{A}\left(\eta_{a_{1}}, \ldots, \eta_{a_{N}}\right) \in \mathrm{PP}_{\mathrm{N}}$. See Figures $4-5$. The goal of this section is to derive the probabilities for $\mathbb{P}[\mathcal{A}=\beta]$.

Theorem 4.1. Fix $\alpha \in \mathrm{PP}_{\mathrm{N}}$. Let $\Gamma+u_{\alpha}$ be the GFF on $\mathrm{H}$ with boundary data given by (4.2). Consider the planar pair partition $\mathcal{A}$ formed by its level lines described as above. Then we have

$$
\mathbb{P}[\mathcal{A}=\beta]=\mathcal{M}_{\alpha, \beta} \frac{\mathcal{Z}_{\beta}\left(x_{1}, \ldots, x_{2 N}\right)}{\mathcal{U}_{\alpha}\left(x_{1}, \ldots, x_{2 N}\right)}, \quad \text { for all } \beta \in \mathrm{PP}_{\mathrm{N}},
$$



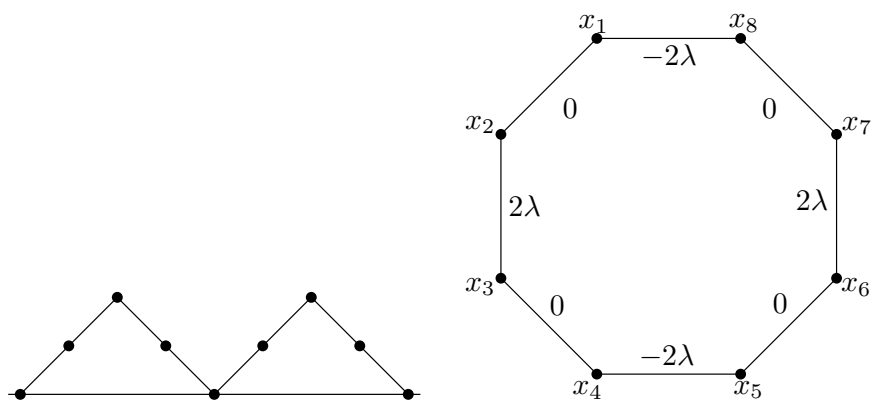

Figure 4: Illustration of the boundary data with $N=4$ : the planar pair partition for boundary data is $\alpha=\{\{1,4\},\{2,3\},\{5,8\},\{6,7\}\}$ as ordered in (2.2).

where $\left\{\mathcal{Z}_{\beta}: \beta \in \mathrm{PP}_{\mathrm{N}}\right\}$ are pure partition functions for multiple $\mathrm{SLE}_{4},\left\{\mathcal{U}_{\alpha}: \alpha \in \mathrm{DP}_{\mathrm{N}}\right\}$ are conformal block functions defined in (3.10), and $\left\{\mathcal{M}_{\gamma, \beta}: \gamma, \beta \in \mathrm{PP}_{\mathrm{N}}\right\}$ is the incidence matrix defined through (3.11).

Theorem 4.1 is a generalization of [PW19, Theorem 1.4] where the authors derive the connection probabilities for $\alpha=\{\{1,2\},\{3,4\}, \ldots,\{2 N-1,2 N\}\}$.
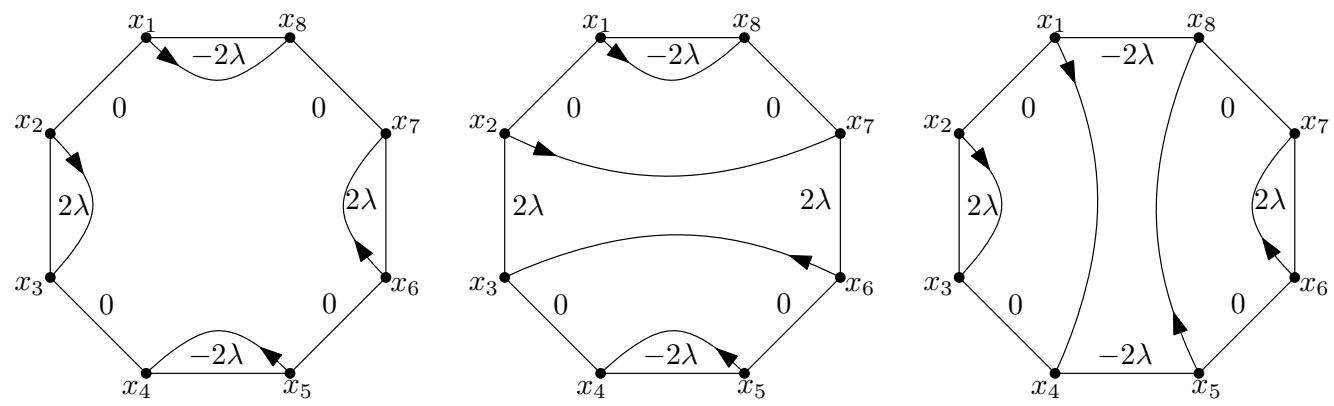

Figure 5: Figure 4 continued: the curve $\eta_{i}$ is the level line starting from $x_{i}$ with height $-\lambda$ for $i=1,5$; the curve $\eta_{i}$ is the level line starting from $x_{i}$ with height $\lambda$ for $i=2,6$. The four curves $\eta_{1}, \eta_{2}, \eta_{5}, \eta_{6}$ connect the eight boundary points. Their end points give a planar pair partition, and there are three possibilities as indicated in the figure. From left to right, the three planar pair partitions are $\beta_{1}=\{\{1,8\},\{2,3\},\{5,4\},\{6,7\}\}$, $\beta_{2}=\{\{1,8\},\{2,7\},\{5,4\},\{6,3\}\}, \beta_{3}=\{\{1,4\},\{2,3\},\{5,8\},\{6,7\}\}$. Note that $\alpha \stackrel{()}{\longleftarrow} \beta_{i}$ for $i=1,2,3$.

Lemma 4.2. Let $\eta=\eta_{1}$ be the level line of $\Gamma+u_{\alpha}$ starting from $x_{1}$ with height $-\lambda$, let $\left(W_{t}, t \geq 0\right)$ be the driving function, and $\left(g_{t}, t \geq 0\right)$ be the corresponding conformal maps, and $T$ be the continuation threshold. For a smooth function $F: \mathfrak{X}_{2 N} \rightarrow \mathbb{R}$, the process

$$
M_{t}:=\frac{F\left(W_{t}, g_{t}\left(x_{2}\right), \ldots, g_{t}\left(x_{2 N}\right)\right)}{\mathcal{U}_{\alpha}\left(W_{t}, g_{t}\left(x_{2}\right), \ldots, g_{t}\left(x_{2 N}\right)\right)}
$$

is a local martingale if and only if $F$ satisfies PDE (3.2) with $\kappa=4$ and $i=1$.

Proof. The level line of $\Gamma+u_{\alpha}$ starting from $x_{1}$ with height $-\lambda$ is the $\operatorname{SLE}_{4}\left(\rho_{2}, \ldots, \rho_{2 \mathrm{~N}}\right)$ process with force points $\left(x_{2}, \ldots, x_{2 N}\right)$ and $\rho_{i}=2(\alpha(i)-\alpha(i-1))$. Recalling from (4.1), its driving function $W_{t}$ satisfies the following intergrated SDEs up to the continuation 


\section{Crossing probabilities in metric graph GFF}

threshold $T$ :

$$
W_{t}=2 B_{t}+x_{1}+\sum_{i=2}^{2 N} \int_{0}^{t} \frac{\rho_{i} d s}{W_{s}-g_{s}\left(x_{i}\right)}, \quad g_{t}\left(x_{i}\right)=x_{i}+\int_{0}^{t} \frac{2 d s}{g_{s}\left(x_{i}\right)-W_{s}}, \quad \text { for } 2 \leq i \leq 2 N .
$$

We denote $\boldsymbol{Y}=\left(W_{t}, g_{t}\left(x_{2}\right), \ldots, g_{t}\left(x_{2 N}\right)\right)$ and $X_{i 1}=g_{t}\left(x_{i}\right)-W_{t}$ for $2 \leq i \leq 2 N$. In this proof, we write $\partial_{i}$ for $\frac{\partial}{\partial x_{i}}$ as there is no ambiguity. We denote the differential operator in (3.2) with $\kappa=4$ and $i=1$ by

$$
\mathcal{D}^{(1)}:=2 \partial_{1}^{2}+\sum_{i=2}^{2 N}\left(\frac{2 \partial_{i}}{x_{i}-x_{1}}-\frac{1}{2\left(x_{i}-x_{1}\right)^{2}}\right) .
$$

By Itô's formula, we have

$$
\begin{aligned}
d F(\boldsymbol{Y}) & =2 \partial_{1} F(\boldsymbol{Y}) d B_{t}+\left(2 \partial_{1}^{2}+\sum_{i=2}^{2 N}\left(\frac{2 \partial_{i}}{X_{i 1}}-\frac{\rho_{i} \partial_{1}}{X_{i 1}}\right)\right) F(\boldsymbol{Y}) d t \\
& =2 \partial_{1} F(\boldsymbol{Y}) d B_{t}+\left(\mathcal{D}^{(1)}+\sum_{i=2}^{2 N}\left(\frac{1}{2 X_{i 1}^{2}}-\frac{\rho_{i} \partial_{1}}{X_{i 1}}\right)\right) F(\boldsymbol{Y}) d t .
\end{aligned}
$$

We also have

$\frac{d \mathcal{U}_{\alpha}(\boldsymbol{Y})}{\mathcal{U}_{\alpha}(\boldsymbol{Y})}=-\sum_{i=2}^{2 N} \frac{\vartheta_{\alpha}(1, i)}{X_{i 1}} d B_{t}+\left(\sum_{i=2}^{2 N} \frac{1+\vartheta_{\alpha}(1, i) \rho_{i}}{2 X_{i 1}^{2}}+\sum_{2 \leq i \neq j \leq 2 N} \frac{\frac{1}{2}\left(\vartheta_{\alpha}(1, j) \rho_{i}+\vartheta_{\alpha}(1, i) \rho_{j}\right)}{2 X_{i 1} X_{j 1}}\right) d t$.

By definition, we have $\vartheta_{\alpha}(1, i) \rho_{i}=2$ for $2 \leq i \leq 2 N$ and $\vartheta_{\alpha}(1, j) \rho_{i}+\vartheta_{\alpha}(1, i) \rho_{j}=4 \vartheta_{\alpha}(i, j)$ for $i \neq j$. Thus

$$
\frac{d \mathcal{U}_{\alpha}(\boldsymbol{Y})}{\mathcal{U}_{\alpha}(\boldsymbol{Y})}=-\sum_{i=2}^{2 N} \frac{\vartheta_{\alpha}(1, i)}{X_{i 1}} d B_{t}+\left(\sum_{i=2}^{2 N} \frac{3}{2 X_{i 1}^{2}}+\sum_{2 \leq i \neq j \leq 2 N} \frac{\vartheta_{\alpha}(i, j)}{X_{i 1} X_{j 1}}\right) d t
$$

Therefore, we have

$$
\begin{aligned}
\frac{d M_{t}}{M_{t}} & =\frac{d F(\boldsymbol{Y})}{F(\boldsymbol{Y})}-\frac{d \mathcal{U}_{\alpha}(\boldsymbol{Y})}{\mathcal{U}_{\alpha}(\boldsymbol{Y})}+4\left(\frac{\partial_{1} \mathcal{U}_{\alpha}(\boldsymbol{Y})}{\mathcal{U}_{\alpha}(\boldsymbol{Y})}\right)^{2} d t-4\left(\frac{\partial_{1} \mathcal{U}_{\alpha}(\boldsymbol{Y})}{\mathcal{U}_{\alpha}(\boldsymbol{Y})}\right)\left(\frac{\partial_{1} F(\boldsymbol{Y})}{F(\boldsymbol{Y})}\right) d t \\
& =\left(\frac{2 \partial_{1} F(\boldsymbol{Y})}{F(\boldsymbol{Y})}-\frac{2 \partial_{1} \mathcal{U}_{\alpha}(\boldsymbol{Y})}{\mathcal{U}_{\alpha}(\boldsymbol{Y})}\right) d B_{t}+\frac{\mathcal{D}^{(1)} F(\boldsymbol{Y})}{F(\boldsymbol{Y})} d t .
\end{aligned}
$$

Thus $M_{t}$ is a local martingale if and only if $F$ satisfies PDE (3.2) with $\kappa=4$ and $i=1$.

Proof of Theorem 4.1. We prove by induction on $N$. We write $\alpha=\left\{\left\{a_{1}, b_{1}\right\}, \ldots,\left\{a_{N}, b_{N}\right\}\right\}$ as ordered in (2.2). We first show the conclusion for $\beta \in \mathrm{PP}_{\mathrm{N}}$ such that $\mathcal{M}_{\alpha, \beta}=1$. There exists $j \in\{1,2 \ldots, 2 N-1\}$ such that $\{j, j+1\} \in \beta$. In this case, we have $\wedge^{j} \in \beta$ and $\diamond_{j} \in \alpha$. If $\wedge^{j} \in \alpha$, we let $\eta=\eta_{j}$ be the level line of $\Gamma+u_{\alpha}$ starting from $x_{j}$ with height $\mathcal{H}_{\alpha}(j)$. If $\vee_{j} \in \alpha$, we let $\eta=\eta_{j+1}$ be the level line of $\Gamma+u_{\alpha}$ starting from $x_{j+1}$ with height $\mathcal{H}_{\alpha}(j+1)$. The second case can be proved in a similar way as the first case. So we only give proof for the first case: we may assume $\wedge^{j} \in \alpha$. Let $\eta=\eta_{j}$ be the level line of $\Gamma+u_{\alpha}$ starting from $x_{j}$ with height $\mathcal{H}_{\alpha}(j)$. Let $\left(W_{t}, t \geq 0\right)$ be the driving function, and $\left(g_{t}, t \geq 0\right)$ be the corresponding conformal maps, and $T$ be the continuation threshold.

Define

$$
M_{t}:=\frac{\mathcal{Z}_{\beta}\left(g_{t}\left(x_{1}\right), \ldots, g_{t}\left(x_{j-1}\right), W_{t}, g_{t}\left(x_{j+1}\right), g_{t}\left(x_{2 N}\right)\right)}{\mathcal{U}_{\alpha}\left(g_{t}\left(x_{1}\right), \ldots, g_{t}\left(x_{j-1}\right), W_{t}, g_{t}\left(x_{j+1}\right), g_{t}\left(x_{2 N}\right)\right)} .
$$



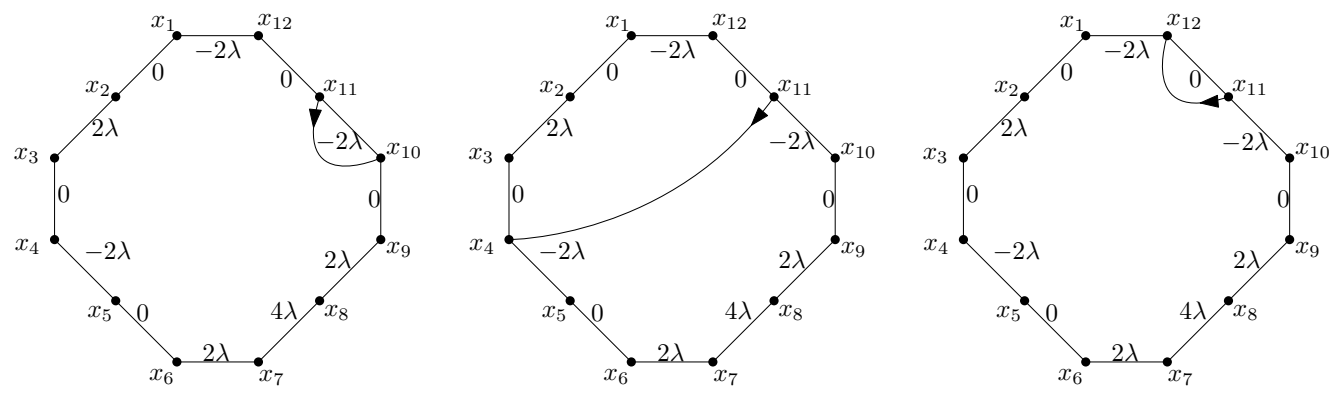

Figure 6: Suppose $\alpha=\{\{1,4\},\{2,3\},\{5,10\},\{6,9\},\{7,8\},\{11,12\}\}$ as ordered in (2.2). We see that $\wedge^{11} \in \alpha$. Consider the level line $\eta_{11}$ starting from $x_{11}$ with height $-\lambda$. It may terminate at $x_{n}$ with positive chance for $n \in\{4,10,12\}$. In all three cases, the boundary data on $\left(x_{n}, x_{n+1}\right)$ is the same as the boundary data on $\left(x_{10}, x_{11}\right)$, and the boundary data on $\left(x_{n-1}, x_{n}\right)$ is the same as the boundary data on $\left(x_{11}, x_{12}\right)$. Therefore, we have $\alpha(n)=\alpha(10)$ and $\alpha(n-1)=\alpha(11)$.

From a similar calculation as in Lemma 4.2, this is a local martingale. From (3.12), this is a bounded martingale. Optional stopping theorem gives $M_{0}=\mathbb{E}\left[M_{T}\right]$. We will analyze the behavior of the process as $t \rightarrow T$. Consider the level line $\eta$, it will terminate at a point $x_{n}$ such that $\alpha(n-1)=\alpha(j)$ and $\alpha(n)=\alpha(j-1)$. See Figure 6 .

If $\eta(T)=x_{j+1}$, from (3.13) and (3.16), we have, as $t \rightarrow T$, almost surely,

$$
\begin{aligned}
M_{t} & =\frac{\left(g_{t}\left(x_{j+1}\right)-W_{t}\right)^{1 / 2} \mathcal{Z}_{\beta}\left(g_{t}\left(x_{1}\right), \ldots, g_{t}\left(x_{j-1}\right), W_{t}, g_{t}\left(x_{j+1}\right), g_{t}\left(x_{2 N}\right)\right)}{\left(g_{t}\left(x_{j+1}\right)-W_{t}\right)^{1 / 2} \mathcal{U}_{\alpha}\left(g_{t}\left(x_{1}\right), \ldots, g_{t}\left(x_{j-1}\right), W_{t}, g_{t}\left(x_{j+1}\right), g_{t}\left(x_{2 N}\right)\right)} \\
& \rightarrow \frac{\mathcal{Z}_{\beta / \wedge_{j}}\left(g_{T}\left(x_{1}\right), \ldots, g_{T}\left(x_{j-1}\right), g_{T}\left(x_{j+2}\right), \ldots, g_{T}\left(x_{2 N}\right)\right)}{\mathcal{U}_{\alpha / \diamond_{j}}\left(g_{T}\left(x_{1}\right), \ldots, g_{T}\left(x_{j-1}\right), g_{T}\left(x_{j+2}\right), \ldots, g_{T}\left(x_{2 N}\right)\right)} .
\end{aligned}
$$

If $\eta(T)=x_{n}$ with $n \neq j+1$, from Lemma A.3 and (3.5), we have $\lim _{t \rightarrow T} M_{t}=0$ almost surely. In summary, we have

$$
M_{0}=\mathbb{E}\left[M_{T}\right]=\mathbb{E}\left[\mathbb{1}_{\left\{\eta(T)=x_{j+1}\right\}} \frac{\mathcal{Z}_{\beta / \wedge_{j}}\left(g_{T}\left(x_{1}\right), \ldots, g_{T}\left(x_{j-1}\right), g_{T}\left(x_{j+2}\right), \ldots, g_{T}\left(x_{2 N}\right)\right)}{\mathcal{U}_{\alpha / \diamond_{j}}\left(g_{T}\left(x_{1}\right), \ldots, g_{T}\left(x_{j-1}\right), g_{T}\left(x_{j+2}\right), \ldots, g_{T}\left(x_{2 N}\right)\right)}\right] .
$$

By induction hypothesis, we have

$$
\mathbb{P}[\mathcal{A}=\beta \mid \eta[0, T]]=\frac{\mathcal{Z}_{\beta / \wedge_{j}}\left(g_{T}\left(x_{1}\right), \ldots, g_{T}\left(x_{j-1}\right), g_{T}\left(x_{j+2}\right), \ldots, g_{T}\left(x_{2 N}\right)\right)}{\mathcal{U}_{\alpha / \diamond_{j}}\left(g_{T}\left(x_{1}\right), \ldots, g_{T}\left(x_{j-1}\right), g_{T}\left(x_{j+2}\right), \ldots, g_{T}\left(x_{2 N}\right)\right)} .
$$

Therefore, $M_{0}=\mathbb{P}[\mathcal{A}=\beta]$ as desired.

The above argument gives (4.4) for $\beta \in \mathrm{PP}_{\mathrm{N}}$ such that $\mathcal{M}_{\alpha, \beta}=1$. Since $\mathcal{U}_{\alpha}=$ $\sum_{\beta} \mathcal{M}_{\alpha, \beta} \mathcal{Z}_{\beta}$, we have

$$
\sum_{\beta: \mathcal{M}_{\alpha, \beta}=1} \mathbb{P}[\mathcal{A}=\beta]=\sum_{\beta: \mathcal{M}_{\alpha, \beta}=1} \frac{\mathcal{Z}_{\beta}}{\mathcal{U}_{\alpha}}=1
$$

This implies $\mathbb{P}[\mathcal{A}=\gamma]=0$ for all $\gamma \in \mathrm{PP}_{\mathrm{N}}$ with $\mathcal{M}_{\alpha, \gamma}=0$. This completes the proof for (4.4).

\section{Metric graph GFF and first passage sets}

In this section, we first introduce discrete GFF and metric graph GFF in Section 5.1, and then we introduce first passage set in Section 5.2. In Section 5.3, we show that 


\section{Crossing probabilities in metric graph GFF}

the crossing probabilities in metric graph GFF converges to the probability of certain connection probabilities in continuum GFF, see Proposition 5.2. This gives the first half of the proof of Theorem 1.1. In order to calculate the desired connection probabilities in continuum GFF, we use Theorem 4.1 and a result about asymptotics of pure partition functions-Proposition 5.6. Section 5.4 proves Proposition 5.6 and Proposition 1.2, and it is quite independent of the rest of the section. Finally, we complete the proof of Theorem 1.1 in Section 5.5.

\subsection{Discrete GFF and metric graph GFF}

In this section, we review basic definition and properties of discrete GFF and metric graph GFF. We refer to [SS09, ALS20] for details. Suppose $\mathcal{G}=(V, E)$ is a connected planar graph, and $\partial \mathcal{G}$ is a given subset of $V$ which we call the boundary of $\mathcal{G}$. Let $\Delta$ be the discrete Laplacian on $\mathcal{G}$ :

$$
(\Delta f)(x)=\sum_{y \sim x}(f(y)-f(x)), \forall x \in V \backslash \partial \mathcal{G} .
$$

The discrete Green's function $G_{\mathcal{G}}$ is the inverse of $-\Delta$ with zero-boundary condition on $\partial \mathcal{G}$. The discrete GFF is the centered Gaussian process $\left(\Gamma^{\mathcal{G}}(v): v \in V\right)$ with covariance given by Green's function:

$$
\mathbb{E}\left[\Gamma^{\mathcal{G}}(x) \Gamma^{\mathcal{G}}(y)\right]=G_{\mathcal{G}}(x, y), \quad \forall x, y \in V .
$$

Suppose $\mathcal{G}=(V, E)$ is a connected planar graph with boundary $\partial \mathcal{G}$. For each $e \in E$, we view it as a line segment in the plane, and for every $x^{\prime}, y^{\prime} \in e$, we define ${ }^{3}$

$$
m\left(\left[x^{\prime}, y^{\prime}\right]\right)=\frac{\left|x^{\prime}-y^{\prime}\right|}{|x-y|} .
$$

This defines a length measure $d m$ on $\mathcal{G}$. We call $(\mathcal{G}, d m)$ metric graph of $\mathcal{G}$ and we denote it by $\tilde{\mathcal{G}}$.

The metric graph GFF $\Gamma^{\tilde{\mathcal{G}}}$ can be constructed as follows, see [Lup16]. First, we sample the discrete $\operatorname{GFF}\left(\Gamma^{\mathcal{G}}(v): v \in V\right)$. Then, conditional on $\left(\Gamma^{\mathcal{G}}(v): v \in V\right)$, for each $e=\{x, y\} \in E$, we sample an independent Brownian bridge with length $m([x, y])$ and two terminal values $\Gamma^{\mathcal{G}}(x)$ and $\Gamma^{\mathcal{G}}(y)$. This defines the metric graph GFF with zero-boundary condition and we denote it by $\left(\Gamma^{\tilde{\mathcal{G}}}(z): z \in \tilde{\mathcal{G}}\right)$. Given a function $u: \partial \mathcal{G} \rightarrow \mathbb{R}$, we choose the discrete harmonic extension of $u$ to $V \backslash \partial \mathcal{G}$ and then extend it inside each edge by linear interpolation. We still denote this function by $u$ and view it as the harmonic function on the metric graph. We call $\Gamma^{\tilde{\mathcal{G}}}+u$ the metric graph GFF with boundary data $u$.

\subsection{First passage sets}

In this section, we introduce first passage sets for metric graph GFF. Suppose $\Gamma^{\tilde{\mathcal{G}}}+u$ is the metric graph GFF with boundary data $u$. For every $a \in \mathbb{R}$, the first passage set above $-a$ is defined by

$\tilde{\mathrm{A}}_{-a}^{u}:=\left\{x \in \tilde{\mathcal{G}} \mid \exists\right.$ a continous path $\gamma$ from $x$ to $\partial \mathcal{G}$ in $\tilde{\mathcal{G}}$ such that $\Gamma^{\tilde{\mathcal{G}}}+u \geq-a$ along $\left.\gamma\right\}$.

Note that, conditional on $\tilde{\mathrm{A}}_{-a}^{u}$, the closure of $\tilde{\mathcal{G}} \backslash \tilde{\mathrm{A}}_{-a}^{u}$ is also a metric graph with length measure inherited from $\tilde{\mathcal{G}}$. According to [ALS20, Proposition 2.1], metric graph GFF satisfies the following space Markov property:

$$
\Gamma^{\tilde{\mathcal{G}}}=\Gamma_{\tilde{\mathbb{A}}_{-a}^{u}}^{\tilde{\mathcal{G}}}+\Gamma^{\tilde{\mathcal{G}}, \tilde{\mathrm{A}}_{-a}^{u},}
$$

\footnotetext{
${ }^{3}$ Here we use the normalization in [ALS20] which is distinct from the one in [Lup16].
} 
where $\Gamma^{\tilde{\mathcal{G}}, \tilde{\mathbb{A}}_{-a}^{u}}$ is the metric graph GFF with zero-boundary condition on the closure of $\tilde{\mathcal{G}} \backslash \tilde{\mathbb{A}}_{-a}^{u}$ conditional on $\tilde{\mathbb{A}}_{-a}^{u}$, and $\Gamma_{\tilde{\mathbb{A}}_{-a}^{u}}^{\tilde{\mathcal{G}}}$ is defined as follows: it is $\Gamma^{\tilde{\mathcal{G}}}$ on $\tilde{\mathbb{A}}_{-a}^{u}$ and it is the harmonic function with boundary value given by $\Gamma^{\tilde{\mathcal{G}}}$ on $\tilde{\mathcal{G}} \backslash \tilde{\mathrm{A}}_{-a}^{u}$.

We also need the following description of first passage set by clusters of loops and excursions. The Brownian loop measure and Brownian excursion measure are conformally invariant measures on Brownian paths in the plane. In this article, we do not need the precise definition of these measures, so we content ourselves with referring their definition to [ALS20, Section 2.2]. We denote by $\mu_{\text {loop }}^{\tilde{\mathcal{G}}}$ the Brownian loop measure on $\tilde{\mathcal{G}}$. Suppose $u$ is non-negative, and we denote by $\mu_{\text {exc }}^{\tilde{\mathcal{G}}, u}$ the Brownian excursion measure on $\tilde{\mathcal{G}}$ with boundary data $u$. We sample Poisson point process with intensity measure $\frac{1}{2} \mu_{\text {loop }}^{\tilde{\mathcal{G}}}$, and denote it by $\mathcal{L}_{1 / 2}^{\tilde{\mathcal{G}}}$. We sample an independent Poisson point process with intensity measure $\mu_{\text {exc }}^{\tilde{\mathcal{G}}, u}$ and denote it by $\Xi_{u}^{\tilde{\mathcal{G}}}$. We denote by $\tilde{\mathcal{A}}\left(\mathcal{L}_{1 / 2}^{\tilde{\mathcal{G}}}, \Xi_{u}^{\tilde{\mathcal{G}}}\right)$ the closure of union of clusters formed by loops and excursions that contain at least one excursion connected to $\partial \mathcal{G}$. As shown in [ALS20, Proposition 2.5], the set $\tilde{\mathcal{A}}\left(\mathcal{L}_{1 / 2}^{\tilde{\mathcal{G}}}, \Xi_{u}^{\tilde{\mathcal{G}}}\right)$ has the same law as the first passage set $\tilde{\mathrm{A}}_{0}^{u}$.

Next, we introduce the first passage set for continuum GFF. To this end, we first introduce local set. Suppose $\Omega \subsetneq \mathbb{C}$ is a simply connected domain and let $\Gamma$ be a continuum GFF on $\Omega$ with zero-boundary condition. We call a random closed set $A \subset \bar{\Omega}$ is a local set of $\Gamma$, if $\Gamma=\Gamma_{A}+\Gamma^{A}$, where $\Gamma_{A}$ and $\Gamma^{A}$ are two random distributions such that $\Gamma_{A}$ is harmonic in $\Omega \backslash A$ and, conditional on $\left(A, \Gamma_{A}\right)$, the function $\Gamma^{A}$ is the GFF with zero-boundary condition in $\Omega \backslash A$. Suppose $h_{A}$ is defined as follows: it is $\Gamma_{A}$ on $\Omega \backslash A$ and it is 0 on $A$. Then we have the following description of the first passage set.

Theorem 5.1. Suppose $\Omega \subsetneq \mathbb{C}$ is a simply connected domain and let $\Gamma$ be a continuum GFF on $\Omega$ with zero-boundary condition. Suppose $u$ is a bounded harmonic function with piecewise constant boundary data. ${ }^{4}$ The first passage set $\mathbb{A}_{-a}^{u}$ is the local set of $\Gamma$ containing $\partial \Omega$ with the following two properties:

- The function $h_{\mathbb{A}_{-a}^{u}}+u$ is harmonic in $\Omega \backslash \mathbb{A}_{-a}^{u}$ such that it equals $-a$ on $\partial \mathbb{A}_{-a}^{u} \backslash \partial \Omega$ and it equals $u$ on $\partial\left(\Omega \backslash \mathbb{A}_{-a}^{u}\right) \cap \partial \Omega$. Moreover, $h_{\mathbb{A}_{-a}^{u}}+u \leq-a$.

- We have $\Gamma_{\mathrm{A}_{-a}^{u}}-h_{\mathrm{A}_{-a}^{u}} \geq 0$. I.e. for any positive smooth function $f$ with compact support, we have $\left(\Gamma_{\mathrm{A}_{-a}^{u}}^{u}-h_{\mathbb{A}_{-a}^{u}}, f\right) \geq 0$.

For all $a \geq 0$, the first passage set $\mathbb{A}_{-a}^{u}$ exists. Moreover, the set $\mathbb{A}_{-a}^{u}$ is the unique local set which satisfies the above two properties and is measurable with respect to $\Gamma$.

Proof. See [ALS20, Theorem 3.5].

Now, we are ready to state the convergence of the first passage set of the metric graph GFF to the first passage set of the continuum GFF. Fix a bounded simply connected domain $\Omega$ such that $\Omega \subset[-C, C]^{2}$ for some $C>0$. Suppose $\left\{\Omega^{\delta}\right\}_{\delta>0}$ is a sequence of simply connected domains such that $\Omega^{\delta} \subset[-C, C]^{2}$ for all $\delta>0$. Suppose $\Omega^{\delta}$ converges to $\Omega$ as $\delta \rightarrow 0$ in the following sense:

$$
[-C, C]^{2} \backslash \Omega^{\delta} \text { converges to }[-C, C]^{2} \backslash \Omega \text { in Hausdorff metric. }
$$

We denote by $\delta \tilde{\mathbb{Z}}^{2}$ the corresponding metric graph. We define $\tilde{\Omega}^{\delta}$ to be the closure of $\Omega^{\delta} \cap \delta \tilde{\mathbb{Z}}^{2}$. It is also a metric graph with metric inherited from $\delta \tilde{\mathbb{Z}}^{2}$. We define its boundary by $\partial \tilde{\Omega}^{\delta}:=\tilde{\Omega}^{\delta} \cap \partial \Omega^{\delta}$. We have the following setup for the convergence.

\footnotetext{
${ }^{4}$ Throughout the article, by piecewise constant boundary data, we mean that the boundary data is piecewise constant and it changes only finitely many times.
} 


\section{Crossing probabilities in metric graph GFF}

- Suppose $\Gamma$ is the continuum GFF on $\Omega$ and $\tilde{\Gamma}^{\delta}$ is the metric grpah GFF on $\tilde{\Omega}^{\delta}$ with zero-boundary condition. We extend $\Gamma$ to $(-C, C)^{2}$ such that it is zero outside $\Omega$, and we still denote the extension by $\Gamma$. We define $\hat{\Gamma}^{\delta}$ on $(-C, C)^{2}$ as follows: it equals $\tilde{\Gamma}^{\delta}$ on $\tilde{\Omega}^{\delta}$ and it is harmonic in $(-C, C)^{2} \backslash \tilde{\Omega}^{\delta}$ which equals zero along $\partial(-C, C)^{2}$.

- Suppose $u$ is a harmonic function on $\Omega$ with piecewise constant boundary data and $u^{\delta}$ is a harmonic function on $\tilde{\Omega}^{\delta}$ for every $\delta>0$ such that $u^{\delta}$ converges to $u$ uniformly as $\delta \rightarrow 0$.

- For $a \in \mathbb{R}$, suppose $\mathbb{A}_{-a}^{u}$ is the first passage set of $\Gamma$ on $\Omega$ and $\tilde{\mathrm{A}}_{-a}^{\delta}$ is the first passage set of $\tilde{\Gamma}^{\delta}$ on $\tilde{\Omega}^{\delta}$. We extend $\Gamma_{\mathrm{A}_{-a}^{u}}$ to $(-C, C)^{2}$ such that it is zero outside $\Omega$, and we still denote the extension by $\Gamma_{\mathrm{A}_{-a}^{u}}^{u}$. We define $\hat{\Gamma}_{\tilde{\mathbb{A}}_{-a}^{u^{\delta}}}^{\delta}$ on $(-C, C)^{2}$ as follows: it equals $\tilde{\Gamma}_{\tilde{\mathrm{A}}_{-a}^{\delta}}^{\delta}$ on $\tilde{\Omega}^{\delta}$ and it is harmonic in $(-C, C)^{2} \backslash \tilde{\Omega}^{\delta}$ which equals zero along $\partial(-C, C)^{2}$.

Proposition 5.2. We have the following convergence in law:

$$
\left(\hat{\Gamma}^{\delta}, \hat{\Gamma}_{\tilde{\mathbb{A}}_{-a}^{u^{\delta}}}^{\delta}, \tilde{\mathrm{A}}_{-a}^{u^{\delta}}\right) \rightarrow\left(\Gamma, \Gamma_{\mathrm{A}_{-a}^{u}}, \overline{\mathbb{A}_{-a}^{u} \cap \Omega}\right), \quad \text { as } \delta \rightarrow 0 .
$$

Furthermore, if we couple $\left\{\hat{\Gamma}^{\delta}\right\}_{\delta>0}$ and $\Gamma$ together such that $\hat{\Gamma}^{\delta} \rightarrow \Gamma$ in probability as distributions on $[-C, C]^{2}$, then $\left(\hat{\Gamma}^{\delta}, \tilde{\mathrm{A}}_{-a}^{\delta}\right) \rightarrow\left(\Gamma, \overline{\mathrm{A}_{-a}^{u} \cap \Omega}\right)$ in probability.

Proof. See [ALS20, Proposition 4.7 and Lemma 4.9].

\subsection{Convergence of the connection probability}

Fix a bounded polygon $\left(\Omega ; y_{1}, \ldots, y_{2 N}\right)$ and suppose $\left(\Omega^{\delta} ; y_{1}^{\delta}, \ldots, y_{2 N}^{\delta}\right)$ converges to $\left(\Omega ; y_{1}, \ldots, y_{2 N}\right)$ as $\delta \rightarrow 0$ in the sense of (1.4). We have the following setup.

- Suppose $\tilde{\Gamma}^{\delta}$ is the zero-boundary metric graph GFF on $\tilde{\Omega}^{\delta}$ and let $u^{\delta}$ be the harmonic function with boundary data (1.5). Suppose $\Gamma$ is zero-boundary GFF on $\Omega$ and let $u$ be the harmonic function with the same boundary data.

- We call the first passage set above 0 of $\tilde{\Gamma}^{\delta}+u^{\delta}$ the positive first passage set and we denote it by $\tilde{\mathrm{A}}^{\delta}$. We call the first passage set above 0 of $-\left(\tilde{\Gamma}^{\delta}+u^{\delta}\right)$ the negative first passage set and we denote it by $\tilde{\mathbb{V}}^{\delta}$. Similarly, we can also define the positive first passage set and the negative first passage set for the continuum GFF in $\Omega$, and we denote them by $\mathbb{A}$ and $\mathbb{V}$ respectively.

Note that the frontier of these first passage sets is a collection of $2 N$ curves connecting the $2 N$ boundary points so that their end points form a planar $2 N$-link pattern of $2 N$ points with index valences $\varsigma=(2, \ldots, 2)$, see Figure 1 . We denote the link pattern by $\mathcal{A}^{\delta}$ for metric graph GFF and by $\mathcal{A}$ for cotinuum GFF. The goal of this section is to prove the following convergence.

Proposition 5.3. Fix $N \geq 1$ and $\varsigma=(2, \ldots, 2)$ of length $2 N$. For all $\hat{\alpha} \in \mathrm{LP}_{\varsigma}$, we have

$$
\lim _{\delta \rightarrow 0} \mathbb{P}\left[\mathcal{A}^{\delta}=\hat{\alpha}\right]=\mathbb{P}[\mathcal{A}=\hat{\alpha}] .
$$

To prove Proposition 5.3, we will give an explicit construction of $\mathbb{A}$ and $\mathbb{V}$ in Lemma 5.4. This construction indicates that the frontier of $\mathbb{A}$ and of $\mathbb{V}$ forms a planar link pattern in $\mathrm{LP}_{\varsigma}$. Then, we prove Lemma 5.5 which indicates that for any subsequence $\delta_{n} \rightarrow 0$ as $n \rightarrow \infty$, there exists a coupling such that the frontier of $\tilde{\mathrm{A}}^{\delta_{n}}$ and of $\tilde{\mathbb{V}}^{\delta_{n}}$ converges to the frontier of $\mathbb{A}$ and of $\mathbb{V}$ almost surely in Hausdorff metric. This indicates the proposition. 


\section{Crossing probabilities in metric graph GFF}

Lemma 5.4. The frontier of $\mathbb{A}$ is the union of level lines of the continuum GFF $\Gamma+u$ starting from $y_{2 j-1}$ with height $\lambda$ for $1 \leq j \leq N$, the frontier of $\mathbb{V}$ is the union of level lines of $\Gamma+u$ starting from $y_{2 j-1}$ with height $-\lambda$ for $1 \leq j \leq N$.

Proof. We will prove the conclusion for $\mathbb{A}$, and the proof for $\mathbb{V}$ is similar. We will argue that the first passage set $\mathbb{A}$ can be constructed as follows. Let $\eta_{j}$ be the level line of $\Gamma+u$ starting from $y_{2 j-1}$ with height $\lambda$ for $1 \leq j \leq N$. Suppose $S_{1}, \ldots, S_{r}$ are the different connected components of $\Omega \backslash\left(\cup_{1 \leq j \leq N} \eta_{j}\right)$ which have $\left(y_{2 j-1}, y_{2 j}\right)$ on their boundary for some $1 \leq j \leq N$. Note that $\left.(\Gamma+u)\right|_{S_{i}}$ has boundary data $2 \lambda$ along $\partial S_{i}$. Conditional on $\cup_{1 \leq j \leq N} \eta_{j}$, we sample the first passage set above zero of $\left.(\Gamma+u)\right|_{S_{i}}$ in each $S_{i}$, and we denote it by $\mathbb{A}_{0, i}^{2 \lambda}$ for $1 \leq i \leq r$. We will show that the union $A:=\left(\cup_{1 \leq j \leq N} \eta_{j}\right) \cup\left(\cup_{1 \leq i \leq r} \mathbb{A}_{0, i}^{2 \lambda}\right) \cup \partial \Omega$ has the same law as $\mathbb{A}$.

First, we prove that $A$ is a local set. By construction,

$$
\begin{aligned}
\Gamma & =\Gamma_{\cup_{1 \leq j \leq N} \eta_{j}}+\Gamma^{\cup_{1 \leq j \leq N} \eta_{j}} \\
& =\Gamma_{\cup_{1 \leq j \leq N} \eta_{j}}+\left.\sum_{i=1}^{r} \Gamma^{\cup_{1 \leq j \leq N} \eta_{j}}\right|_{S_{i}} \\
& =\Gamma_{\cup_{1 \leq j \leq N} \eta_{j}}+\sum_{i=1}^{r}\left(\left.\Gamma^{\cup_{1 \leq j \leq N} \eta_{j}}\right|_{S_{i}}\right)_{\mathrm{A}_{0, i}^{2 \lambda}}+\sum_{i=1}^{r}\left(\left.\Gamma^{\cup_{1 \leq j \leq N} \eta_{j}}\right|_{S_{i}}\right)^{\mathrm{A}_{0, i}^{2 \lambda}} .
\end{aligned}
$$

Note that $\Gamma_{A}:=\Gamma_{\cup_{1 \leq j \leq N} \eta_{j}}+\sum_{i=1}^{r}\left(\left.\Gamma^{\cup_{1 \leq j \leq N} \eta_{j}}\right|_{S_{i}}\right)_{\mathbb{A}_{0, i}^{2 \lambda}}$ is harmonic in $\Omega \backslash A$. Conditional on $\left(A, \Gamma_{A}\right)$, the function $\Gamma^{A}=\sum_{i=1}^{r}\left(\left.\Gamma^{\cup_{1 \leq j \leq N} \eta_{j}}\right|_{S_{i}}\right)^{\mathbb{A}_{0, i}^{2 \lambda}}$ is the continuum GFF with zeroboundary condition in $\Omega \backslash A$. This implies that $A$ is a local set.

Next, we check the two properties in Theorem 5.1. The first one is obvious by construction. For the second one, suppose $f$ is a positive smooth function with compact support in $\Omega$, it suffices to prove

$$
\left(\Gamma_{\cup_{1 \leq j \leq N} \eta_{j}}, f\right)=\left(h_{\cup_{1 \leq j \leq N} \eta_{j}}, f\right)
$$

and

$$
\left(\left(\left.\Gamma^{\cup_{1 \leq j \leq N} \eta_{j}}\right|_{S_{i}}\right)_{\mathbb{A}_{0, i}^{2 \lambda}}+2 \lambda \mathbb{1}_{S_{i}}, f\right) \geq 0 .
$$

Eq. (5.1) is a consequence of properties of level lines of GFF. For (5.2), consider the metric graph $\tilde{S}_{i}^{\delta}=S_{i} \cap \delta \tilde{\mathbb{Z}}^{2}$, we denote by $\left.\tilde{\Gamma}^{\delta}\right|_{\tilde{S}_{i}^{\delta}}$ the metric graph GFF with zeroboundary condition on $\tilde{S}_{i}^{\delta}$. Then by Proposition 5.2, we can couple $\left\{\left(\left.\hat{\Gamma}^{\delta}\right|_{\tilde{S}_{i}^{\delta}}\right)_{\tilde{\mathrm{A}}_{0}^{2 \lambda}}\right\}_{\delta>0}$ and $\left(\left.\Gamma^{\cup_{1 \leq j \leq N} \eta_{j}}\right|_{S_{i}}\right)_{\mathbb{A}_{0, i}^{2 \lambda}}$ together such that

$$
\begin{aligned}
\left(\left(\left.\Gamma^{\cup_{1 \leq j \leq N} \eta_{j}}\right|_{S_{i}}\right)_{\mathbb{A}_{0, i}^{2 \lambda}}+2 \lambda \mathbb{1}_{S_{i}}, f\right) & =\left(\left(\left.\Gamma^{\cup_{1 \leq j \leq N} \eta_{j}}\right|_{S_{i}}\right)_{\mathbb{A}_{0, i}^{2 \lambda}}+2 \lambda \mathbb{1}_{S_{i}}, f \mathbb{1}_{S_{i}}\right) \\
& =\lim _{\delta \rightarrow 0}\left(\left(\left.\hat{\Gamma}^{\delta}\right|_{\tilde{S}_{i}^{\delta}}\right)_{\tilde{\mathbb{A}}_{0}^{2 \lambda}}+2 \lambda \mathbb{1}_{S_{i}}, f \mathbb{1}_{S_{i}}\right) \geq 0 .
\end{aligned}
$$

This gives (5.2). Combining with (5.1) and Theorem 5.1, we see that $A$ has the same law as $\mathbb{A}$, and this completes the proof.

Lemma 5.5. Suppose $\delta_{n} \rightarrow 0$ as $n \rightarrow \infty$.

- Suppose $\left(\mathbb{A}_{1}, \ldots, \mathbb{A}_{r}\right)$ are different connected components of $\overline{\mathbb{A} \cap \Omega}$ and $\left(y_{i, 1} \ldots, y_{i, k_{i}}\right)$ are the marked points on the boundary of $\mathbb{A}_{i}$ for each $1 \leq i \leq r$; and suppose $\left(\mathbb{V}_{1}, \ldots, \mathbb{V}_{s}\right)$ are different connected components of $\overline{\mathbb{V} \cap \Omega}$ and $\left(y_{j, 1}, \ldots, y_{j, l_{j}}\right)$ are the marked points on the boundary of $\mathbb{\forall}_{j}$ for each $1 \leq j \leq s$. 


\section{Crossing probabilities in metric graph GFF}

- Suppose $\left(\tilde{\mathrm{A}}_{1}^{\delta_{n}}, \ldots, \tilde{\mathrm{A}}_{r_{n}}^{\delta_{n}}\right)$ are the connected components of

$$
\tilde{\mathrm{A}}^{\delta_{n}} \cup\left(\cup_{1 \leq l \leq N}\left(y_{2 l-1}^{\delta_{n}}, y_{2 l}^{\delta_{n}}\right)\right) ;
$$

and suppose $\left(\tilde{\mathbb{V}}_{1}^{\delta_{n}}, \ldots, \tilde{\mathbb{V}}_{s_{n}}^{\delta_{n}}\right)$ are the connected components of

$$
\tilde{\mathbb{V}}^{\delta_{n}} \cup\left(\cup_{1 \leq l \leq N}\left(y_{2 l}^{\delta_{n}}, y_{2 l+1}^{\delta_{n}}\right)\right) .
$$

Then there exists a coupling of $\left\{\left(\tilde{\mathbb{A}}^{\delta_{n}}, \tilde{\mathbb{V}}^{\delta_{n}}\right)\right\}_{n \geq 1}$ and $(\mathbb{A}, \mathbb{V})$ such that the following holds almost surely.

- For $n$ large enough, we have $r_{n}=r$ and $s_{n}=s$.

- Moreover, we can reorder $\left(\tilde{\mathbb{A}}_{1}^{\delta_{n}}, \ldots, \tilde{\mathrm{A}}_{r}^{\delta_{n}}\right)$ and we still denote them by $\left(\tilde{\mathbb{A}}_{1}^{\delta_{n}}, \ldots, \tilde{\mathbb{A}}_{r}^{\delta_{n}}\right)$, such that $\left(y_{i, 1}^{\delta_{n}}, \ldots, y_{i, k_{i}}^{\delta_{n}}\right)$ are the marked points on the boundary of $\tilde{\mathbb{A}}_{i}^{\delta_{n}}$ for $1 \leq$ $i \leq r$. Similarly, we can reorder $\left(\tilde{\mathbb{V}}_{1}^{\delta_{n}}, \ldots, \tilde{\mathbb{V}}_{s}^{\delta_{n}}\right)$ and we still denote them by $\left(\tilde{\mathbb{V}}_{1}^{\delta_{n}}, \ldots, \tilde{\mathbb{V}}_{s}^{\delta_{n}}\right)$ such that $\left(y_{j, 1}^{\delta_{n}}, \ldots, y_{j, l_{j}}^{\delta_{n}}\right)$ are the marked points on the boundary of $\tilde{\mathbb{V}}_{j}^{\delta_{n}}$ for $1 \leq j \leq s$.

- Furthermore, we have that $\tilde{\mathrm{A}}_{i}^{\delta_{n}}$ converges to $\mathbb{A}_{i}$ for each $1 \leq i \leq r$ and $\tilde{\mathbb{V}}_{j}^{\delta_{n}}$ converges to $\mathbb{V}_{j}$ for each $1 \leq j \leq s$ in Hausdorff metric.
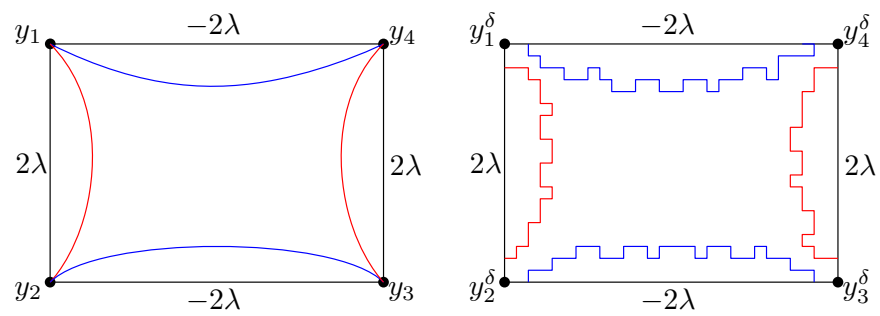

Figure 7: Suppose $N=2$. The frontier of the first passage sets $\mathbb{A}$ and $\mathbb{V}$ is a collection of 4 curves connecting the four points $y_{1}, y_{2}, y_{3}, y_{4}$. There are three possibilities for the connectivity patterns as indicated in Figure 1. In this figure, in the left panel, we have the first possibility. The two red curves are frontiers of $\mathbb{A}_{1}$ and $\mathbb{A}_{2}$ and the two blue curves are frontiers of $\mathbb{V}_{1}$ and $\mathbb{V}_{2}$. In the right panel, we are in metric graph and the two red curves are frontiers of $\mathbb{A}_{1}^{\delta_{n}}$ and $\mathbb{A}_{2}^{\delta_{n}}$ and the two blue curves are frontiers of $\mathbb{V}_{1}^{\delta_{n}}$ and $\mathbb{V}_{2}^{\delta_{n}}$. Lemma 5.5 guarantees that there exists a coupling such that, when $\delta_{n} \rightarrow 0$, the red curves in the right panel converges to the red curves in the left panel and the blue curves in the right panel converges to the blue curves in the left panel respectively.

Proof. We denote by $\tilde{R}_{i}^{\delta_{n}}$ the connected component of $\tilde{\mathbb{A}}^{\delta_{n}} \cup\left(\cup_{1 \leq l \leq N}\left(y_{2 l-1}^{\delta_{n}}, y_{2 l}^{\delta_{n}}\right)\right)$ which contains $\left(y_{2 i-1}^{\delta_{n}}, y_{2 i}^{\delta_{n}}\right)$ on its boundary for $1 \leq i \leq N$; and we denote by $\tilde{S}_{i}^{\delta_{n}}$ the connected component of $\tilde{\mathbb{V}}^{\delta_{n}} \cup\left(\cup_{1 \leq l \leq N}\left(y_{2 l}^{\delta_{n}}, y_{2 l+1}^{\delta_{n}}\right)\right)$ which contains $\left(y_{2 i}^{\delta_{n}}, y_{2 i+1}^{\delta_{n}}\right)$ on its boundary for $1 \leq i \leq N$. Note that the sequence

$$
\left\{\left(\tilde{R}_{1}^{\delta_{n}}, \ldots, \tilde{R}_{N}^{\delta_{n}}, \tilde{\mathbb{A}}^{\delta_{n}}, \tilde{S}_{1}^{\delta_{n}}, \ldots, \tilde{S}_{N}^{\delta_{n}}, \tilde{\mathbb{V}}^{\delta_{n}}\right)\right\}_{n \geq 1}
$$




\section{Crossing probabilities in metric graph GFF}

is tight. Thus, it suffices to prove that Lemma 5.5 holds for any convergent subsequence. Given any convergent subsequence, we still denote it by

$$
\left\{\left(\tilde{R}_{1}^{\delta_{n}}, \ldots, \tilde{R}_{N}^{\delta_{n}}, \tilde{\mathrm{A}}^{\delta_{n}}, \tilde{S}_{1}^{\delta_{n}}, \ldots, \tilde{S}_{N}^{\delta_{n}}, \tilde{\mathbb{V}}^{\delta_{n}}\right)\right\}_{n \geq 1} .
$$

By Skorokhod representation theorem, we can couple them on the same probability space such that there is almost sure convergence. We denote the probability measure of this coupling by $\mathbb{P}$, and we denote its limit by $\left(R_{1}, \ldots, R_{N}, R, S_{1}, \ldots, S_{N}, S\right)$.

By Proposition 5.2, we know that $R=\overline{\mathbb{A} \cap \Omega}$ and $S=\overline{\mathbb{V} \cap \Omega}$. We will prove that $R_{i}$ is the connected component of $\mathbb{A}$ which contains $\left(y_{2 i-1}, y_{2 i}\right)$ on its boundary and $S_{i}$ is the connected component of $\mathbb{V}$ which contains $\left(y_{2 i}, y_{2 i+1}\right)$ on its boundary. Moreover, we will show that Lemma 5.5 holds in this coupling. We only give proof for the positive first passage set, as the proof for the negative first passage set is similar. The proof is divided into two steps. First, suppose $\left(y_{i_{1}}, \ldots, y_{i_{k}}\right)$ are the marked points on the boundary of $R_{i}$. We will prove that $\left(y_{i_{1}}^{\delta_{n}}, \ldots, y_{i_{k}}^{\delta_{n}}\right)$ are the marked points on the boundary of $\tilde{R}_{i}^{\delta_{n}}$ for $n$ large enough. Then we will prove that $R_{i}$ is the connected component of $R$ which contains $\left(y_{2 i-1}, y_{2 i}\right)$ on its boundary for each $1 \leq i \leq N$. See Figure 7 .

For the first step, it is clear that if $y_{j} \notin R_{i}$ for some $1 \leq j \leq 2 N$, we have $y_{j}^{\delta_{n}} \notin \tilde{R}_{i}^{\delta_{n}}$ for $n$ large enough by the almost sure convergence. For $1 \leq j \leq 2 N$, we define the event

$$
F_{j}:=\left\{y_{j} \in R_{i}, \text { but } y_{j}^{\delta_{n}} \notin \tilde{R}_{i}^{\delta_{n}} \text { except for finitely many } n\right\} .
$$

It suffices to prove $\mathbb{P}\left[F_{j}\right]=0$. By Lemma 5.4, we have $S \cap\left(y_{2 i-1}, y_{2 i}\right)=\emptyset$ for $1 \leq i \leq N$. We denote by $D_{i}$ the connected component of $\Omega \backslash S$ which contains $\left(y_{2 i-1}, y_{2 i}\right)$ and we denote by $D_{i}^{\delta_{n}}$ the connected component of $\Omega^{\delta_{n}} \backslash \tilde{\mathbb{V}}^{\delta_{n}}$ which contains $\left(y_{2 i-1}^{\delta_{n}}, y_{2 i}^{\delta_{n}}\right)$. By Carathéodory kernel theorem, the domain $D_{i}^{\delta_{n}}$ converges to $D_{i}$ in Carathéodory topology as $n \rightarrow \infty$. Note that $\tilde{\mathrm{A}}^{\delta_{n}} \cap \tilde{D}_{i}^{\delta_{n}}$ is the first passage set $\tilde{\mathrm{A}}_{0}^{v^{\delta_{n}}}$ of the metric graph GFF on $\tilde{D}_{i}^{\delta_{n}}$ with boundary data given by $v^{\delta_{n}}$ which is defined as follows: $v^{\delta_{n}}$ equals 0 on $\tilde{\mathbb{V}}^{\delta_{n}} \cap \partial \tilde{D}_{i}^{\delta_{n}}$ and $v^{\delta_{n}}$ equals $2 \lambda$ on $\partial \tilde{\Omega}^{\delta_{n}} \cap \partial \tilde{D}_{i}^{\delta_{n}}$. We may assume $j$ is odd. On the event $F_{j}$, we have $\left(y_{j}, y_{j+1}\right) \subset \partial D_{i}$. Thus for $n$ large enough, we have $\left(y_{j}^{\delta_{n}}, y_{j+1}^{\delta_{n}}\right) \subset \partial D_{i}^{\delta_{n}}$. In such case, we define the harmonic function $v_{1}^{\delta_{n}}$ on $\tilde{D}_{i}^{\delta_{n}}$ as follows: it equals 0 on $\left(y_{j}^{\delta_{n}}, y_{j+1}^{\delta_{n}}\right)$ and it equals $v^{\delta_{n}}$ on $\partial \tilde{D}_{i}^{\delta_{n}} \backslash\left(y_{j}^{\delta_{n}}, y_{j+1}^{\delta_{n}}\right)$. Then, in the construction of $\tilde{\mathbb{A}}_{0}^{v^{\delta_{n}}}$ by loops and excursions, we can divide the excursions into two independent parts: the excursions connecting to $\left(y_{j}^{\delta_{n}}, y_{j+1}^{\delta_{n}}\right)$ and the excursions which do not intersect $\left(y_{j}^{\delta_{n}}, y_{j+1}^{\delta_{n}}\right)$. Note that the excursions connecting to $\left(y_{j}^{\delta_{n}}, y_{j+1}^{\delta_{n}}\right)$ correspond to the Poisson point process with intensity measure $\mu_{\operatorname{exc}}^{\tilde{D}_{n}^{\delta_{n}}, v^{\delta_{n}}}-\mu_{\text {exc }}^{\tilde{D}_{\delta_{n}}^{\delta_{n}}, v_{1}^{\delta_{n}}}$ and that the excursions which do not intersect $\left(y_{j}^{\delta_{n}}, y_{j+1}^{\delta_{n}}\right)$ correspond to the Poisson point process with intensity measure $\mu_{\operatorname{exc}}^{\tilde{D}_{i}^{\delta_{n}}, v_{1}^{\delta_{n}}}$. Thus, we have $\tilde{R}_{i}^{\delta_{n}} \subset \tilde{\mathcal{A}}\left(\mathcal{L}_{1 / 2}^{\tilde{D}_{i}^{\delta_{n}}}, \Xi_{v_{1}^{\delta_{n}}}^{\tilde{D}_{i}^{\delta_{n}}}\right)$ if $y_{j}^{\delta_{n}} \notin \tilde{R}_{i}^{\delta_{n}}$. Note that $\tilde{\mathrm{A}}_{0}^{v_{1}^{\delta_{n}}}$ has the same law as $\tilde{\mathcal{A}}\left(\mathcal{L}_{1 / 2}^{\tilde{D}_{i}^{\delta_{n}}}, \Xi_{v_{1}^{\delta_{n}}}^{\tilde{D}_{i}^{\delta_{n}}}\right)$. According to [ALS20, Corollary 4.12], the limit of $\overline{\tilde{\mathbb{A}}_{0}^{v_{1}^{\delta_{n}}} \cap D_{i}}$ does not intersect $\left(y_{j}, y_{j+1}\right)$ almost surely. This implies $\mathbb{P}\left[F_{j}\right]=0$.

For the second step, we define the event $F_{i, k}:=\left\{R_{i} \neq R_{k}\right.$, but $\left.R_{i} \cap R_{k} \neq \emptyset\right\}$ for $1 \leq i<k \leq N$. It suffices to prove that $\mathbb{P}\left[F_{i, k}\right]=0$. Note that on the event $F_{i, k}$, we have $\left(y_{2 k-1}, y_{2 k}\right) \subset D_{i, k}$. This implies $\left(y_{2 k-1}^{\delta_{n}}, y_{2 k}^{\delta_{n}}\right) \subset D_{i, k}^{\delta_{n}}$ for $n$ large enough. Moreover, we have $\tilde{R}_{i}^{\delta_{n}} \cap \tilde{R}_{k}^{\delta_{n}}=\emptyset$ for $n$ large enough. This implies $\tilde{R}_{i}^{\delta_{n}} \cap\left(y_{2 k-1}^{\delta_{n}}, y_{2 k}^{\delta_{n}}\right)=\emptyset$. We denote by $D_{i, k}$ the connected component of $D_{i} \backslash R_{i}$ with $\left(y_{2 k-1}, y_{2 k}\right)$ on its boundary and we denote by $D_{i, k}^{\delta_{n}}$ the connected component of $D_{i}^{\delta_{n}} \backslash \tilde{R}_{i}^{\delta_{n}}$ with $\left(y_{2 k-1}^{\delta_{n}}, y_{2 k}^{\delta_{n}}\right)$ on its 
boundary. Then by Carathéodory kernel theorem, the domain $D_{i, k}^{\delta_{n}}$ converges to $D_{i, k}$ as $n \rightarrow \infty$ in Carathéodory topology. Note that $\tilde{\mathrm{A}}^{\delta_{n}} \cap \tilde{D}_{i, k}^{\delta_{n}}$ is the first passage set of the metric graph GFF with boundary data $w^{\delta_{n}}$ on $\tilde{D}_{i, k}^{\delta_{n}}$, where $w^{\delta_{n}}$ is defined as follows: $w^{\delta_{n}}$ equals 0 on $\left(\tilde{\mathbb{V}}^{\delta_{n}} \cup R_{i}^{\delta_{n}}\right) \cap \partial \tilde{D}_{i, k}^{\delta_{n}}$ and $w^{\delta_{n}}$ equals $2 \lambda$ on $\partial \tilde{\Omega}^{\delta_{n}} \cap \partial \tilde{D}_{i, k}^{\delta_{n}}$. According to [ALS20, Corollary 4.12], the limit of $\tilde{\mathrm{A}}_{0}^{w_{n}^{\delta_{n}} \cap D_{i, k}}$ does not intersect $\left(y_{2 i-2}, y_{2 i-1}\right) \cup \partial R_{i} \cup\left(y_{2 i}, y_{2 i+1}\right)$. This implies $\mathbb{P}\left[F_{i, k}\right]=0$. It completes the proof.

\subsection{Asymptotics of partition functions and proof of Proposition 1.2}

In this subsection, we will give the following asymptotics of pure partition functions: Proposition 5.6. The purpose of this proposition will be clear in the proof of Theorem 1.1.

Proposition 5.6. Fix $\kappa=4$. Fix $N \geq 1$ and the index valences $\varsigma=(2, \ldots, 2)$ of length $2 N$. For each $\hat{\alpha} \in \mathrm{LP}_{\varsigma}$, let $\alpha:=\tau(\hat{\alpha}) \in \mathrm{PP}_{2 \mathrm{~N}}$ be the associated planar pair partition as defined in Section 2.2, and $\mathcal{Z}_{\alpha}$ be the pure partition function associated to $\alpha$. Then, the following limit exists: for $y_{1}<\cdots<y_{2 N}$ and $x_{1}<\cdots<x_{4 N}$,

$$
\mathcal{Z}_{\hat{\alpha}}\left(y_{1}, \ldots, y_{2 N}\right):=\lim _{\substack{x_{2 j-1}, x_{2 j} \rightarrow y_{j} \\ \forall 1 \leq j \leq 2 N}} \frac{\mathcal{Z}_{\alpha}\left(x_{1}, \ldots, x_{4 N}\right)}{\prod_{j=1}^{2 N}\left(x_{2 j}-x_{2 j-1}\right)^{1 / 2}} .
$$

We will show Proposition 5.6 by the explicit expression for $\mathcal{Z}_{\alpha}$ from (3.12):

$$
\mathcal{Z}_{\alpha}\left(x_{1}, \ldots, x_{4 N}\right)=\sum_{\beta \in \mathrm{DP}_{2 \mathrm{~N}}} \mathcal{M}_{\alpha, \beta}^{-1} \mathcal{U}_{\beta}\left(x_{1}, \ldots, x_{4 N}\right) .
$$

For $\beta \in \mathrm{DP}_{2 \mathrm{~N}}$ such that $\times_{2 j-1} \in \beta$ for all $1 \leq j \leq 2 N$, it is easy to see that $\mathcal{U}_{\beta}$ admits a limit when normalized by $\prod_{j}\left(x_{2 j}-x_{2 j-1}\right)^{1 / 2}$, see Lemma 5.7. However, for other $\beta$, the conformal block $\mathcal{U}_{\beta}$ explodes when normalized by $\prod_{j}\left(x_{2 j}-x_{2 j-1}\right)^{1 / 2}$. In order to derive the existence of the limit, we need to group distinct $\beta$ 's properly so that the explosion cancels. The proof of Proposition 5.6 involves heavy notation which we find unavoidable. As the proof is lengthy and not instructive to include in the main text, we put it in Appendix B. We suggest readers to first read the proof of Corollary 5.10 where we give the proof for Proposition 5.6 when $N=2$.

Lemma 5.7. Fix $\kappa=4$. Fix $N \geq 1$. Given a Dyck path $\beta \in \mathrm{DP}_{2 \mathrm{~N}}$ of length $4 N$ such that $\times_{2 j-1} \in \beta$ for all $1 \leq j \leq 2 N$, define $(\beta)_{2} \in \mathrm{DP}_{\mathrm{N}}$ by

$$
(\beta)_{2}(k)=\frac{1}{2} \beta(2 k), \quad 1 \leq k \leq 2 N .
$$

One may check that this is a well-defined Dyck path of length $2 N$. Then, for $y_{1}<\cdots<y_{2 N}$ and $x_{1}<\cdots<x_{4 N}$, we have

$$
\lim _{\substack{x_{2 j-1}, x_{2 j} \rightarrow y_{j} \\ \forall 1 \leq j \leq 2 N}} \frac{\mathcal{U}_{\beta}\left(x_{1}, \ldots, x_{4 N}\right)}{\prod_{j=1}^{2 N}\left(x_{2 j}-x_{2 j-1}\right)^{1 / 2}}=\mathcal{U}_{(\beta)_{2}}^{4}\left(y_{1}, \ldots, y_{2 N}\right) .
$$

Proof. By the definition (3.10), we have

$$
\begin{aligned}
& \frac{\mathcal{U}_{\beta}\left(x_{1}, \ldots, x_{4 N}\right)}{\prod_{j=1}^{2 N}\left(x_{2 j}-x_{2 j-1}\right)^{1 / 2}} \\
= & \prod_{1 \leq s<t \leq 2 N}\left(\left(x_{2 t}-x_{2 s}\right)\left(x_{2 t}-x_{2 s-1}\right)\left(x_{2 t-1}-x_{2 s}\right)\left(x_{2 t-1}-x_{2 s-1}\right)\right)^{\frac{1}{2} \vartheta_{\beta}(2 s, 2 t)} .
\end{aligned}
$$

Thus

$$
\lim _{\substack{x_{2 j-1, x_{2 j} \rightarrow y_{j}}, \forall 1 \leq j \leq 2 N}} \frac{\mathcal{U}_{\beta}\left(x_{1}, \ldots, x_{4 N}\right)}{\prod_{j=1}^{2 N}\left(x_{2 j}-x_{2 j-1}\right)^{1 / 2}}=\prod_{1 \leq s<t \leq 2 N}\left(y_{t}-y_{s}\right)^{2 \vartheta_{\beta}(2 t, 2 s)}=\mathcal{U}_{(\beta)_{2}}^{4}\left(y_{1}, \ldots, y_{2 N}\right) .
$$


Assuming Proposition 5.6 holds, we will extend definition of $\mathcal{Z}_{\hat{\alpha}}$ via conformal covariance.

Corollary 5.8. Assume the same notations as in Proposition 5.6. The function $\mathcal{Z}_{\hat{\alpha}}$ satisfies the following conformal covariance: for all Möbius maps $\varphi$ of $\mathbb{H}$ such that $\varphi\left(y_{1}\right)<\cdots<\varphi\left(y_{2 N}\right)$, we have

$$
\mathcal{Z}_{\hat{\alpha}}\left(y_{1}, \ldots, y_{2 N}\right)=\prod_{i=1}^{2 N} \varphi^{\prime}\left(y_{i}\right) \times \mathcal{Z}_{\hat{\alpha}}\left(\varphi\left(y_{1}\right), \ldots, \varphi\left(y_{2 N}\right)\right) .
$$

For general polygon $\left(\Omega ; y_{1}, \ldots, y_{2 N}\right)$, we define

$$
\mathcal{Z}_{\hat{\alpha}}\left(\Omega ; y_{1}, \ldots, y_{2 N}\right):=\prod_{i=1}^{2 N} \varphi^{\prime}\left(y_{i}\right) \times \mathcal{Z}_{\hat{\alpha}}\left(\varphi\left(y_{1}\right), \ldots, \varphi\left(y_{2 N}\right)\right),
$$

where $\varphi$ is any conformal map from $\Omega$ onto $\mathbb{H}$ such that $\varphi\left(y_{1}\right)<\ldots<\varphi\left(y_{2 N}\right)$.

Proof. The conformal covariance (5.5) is a consequence of (3.3) and the existence of the limit (5.3). From (5.5), we see that (5.6) is well-defined: suppose $\varphi_{1}$ and $\varphi_{2}$ are conformal maps on $\Omega$ with $\varphi_{n}\left(y_{1}\right)<\cdots<\varphi_{n}\left(y_{2 N}\right)$ for $n=1$, 2. From (5.5), we have

$$
\prod_{i=1}^{2 N} \varphi_{1}^{\prime}\left(y_{i}\right) \times \mathcal{Z}_{\hat{\alpha}}\left(\varphi_{1}\left(y_{1}\right), \ldots, \varphi_{1}\left(y_{2 N}\right)\right)=\prod_{i=1}^{2 N} \varphi_{2}^{\prime}\left(y_{i}\right) \times \mathcal{Z}_{\hat{\alpha}}\left(\varphi_{2}\left(y_{1}\right), \ldots, \varphi_{2}\left(y_{2 N}\right)\right) .
$$

Assuming Proposition 5.6 holds, we are able to complete the proof of (1.6) and (1.8). Proof of (1.6). This is immediate from Corollary 5.8.

Proof of Proposition 1.2. We will show (1.8) with $j=1$, and the other cases can be proved similarly. For $y_{1}<\cdots<y_{2 N}$, we denote $y_{k l}=y_{k}-y_{l}$ for $k \neq l$. For $x_{1}<\cdots<x_{4 N}$, we denote $x_{k l}=x_{k}-x_{l}$ for $k \neq l$.

Fix $N \geq 1$ and the index valences $\varsigma=(2, \ldots, 2)$ of length $2 N$. Fix $\hat{\alpha} \in \mathrm{LP}_{\varsigma}$ and let $\alpha=\tau(\hat{\alpha}) \in \mathrm{PP}_{2 \mathrm{~N}}$ be the associated planar pair partition as defined in Section 2.2. We set $F_{0}\left(x_{1}, \ldots, x_{4 N}\right)=\mathcal{Z}_{\alpha}\left(x_{1}, \ldots, x_{4 N}\right)$ for $x_{1}<\cdots<x_{4 N}$. We define $F_{j}$ by induction on $j$. Fix $j \in\{1,2, \ldots, 2 N\}$ and suppose $F_{j-1}$ is defined. For $y_{1}<\cdots<y_{j}<x_{2 j+1}<\cdots<x_{4 N}$ and $y_{j-1}<x_{2 j-1}<x_{2 j}<x_{2 j+1}$, we define

$$
F_{j}\left(y_{1}, \ldots, y_{j}, x_{2 j+1}, \ldots, x_{4 N}\right):=\lim _{x_{2 j-1}, x_{2 j} \rightarrow y_{j}} \frac{F_{j-1}\left(y_{1}, \ldots, y_{j-1}, x_{2 j-1}, x_{2 j}, \ldots, x_{4 N}\right)}{\left(x_{2 j}-x_{2 j-1}\right)^{1 / 2}} .
$$

From Proposition 5.6, we see that $F_{1}, \ldots, F_{2 N}$ are well-defined and $F_{2 N}=\mathcal{Z}_{\hat{\alpha}}$. We will show the following PDE by induction on $j \in\{1,2, \ldots, 2 N\}$ :

$$
\mathcal{D}_{j} F_{j}\left(y_{1}, \ldots, y_{j}, x_{2 j+1}, \ldots, x_{4 N}\right)=0,
$$

where

$$
\begin{aligned}
\mathcal{D}_{j}=\frac{\partial^{3}}{\partial y_{1}^{3}} & -4\left(\sum_{2 \leq i \leq j}\left(\frac{1}{y_{i 1}^{2}}-\frac{1}{y_{i 1}} \frac{\partial}{\partial y_{i}}\right)+\sum_{2 j+1 \leq i \leq 4 N}\left(\frac{1 / 4}{\left(x_{i}-y_{1}\right)^{2}}-\frac{1}{\left(x_{i}-y_{1}\right)} \frac{\partial}{\partial x_{i}}\right)\right) \frac{\partial}{\partial y_{1}} \\
& +2\left(\sum_{2 \leq i \leq j}\left(\frac{2}{y_{i 1}^{3}}-\frac{1}{y_{i 1}^{2}} \frac{\partial}{\partial y_{i}}\right)+\sum_{2 j+1 \leq i \leq 4 N}\left(\frac{1 / 2}{\left(x_{i}-y_{1}\right)^{3}}-\frac{1}{\left(x_{i}-y_{1}\right)^{2}} \frac{\partial}{\partial x_{i}}\right)\right) .
\end{aligned}
$$




\section{Crossing probabilities in metric graph GFF}

When $j=1$, PDE (5.7) holds due to (3.8) with $j=1$. For $j \geq 2$, suppose (5.7) holds for $j-1$, and we will show it for $j$. Comparing the two operators $\mathcal{D}_{j-1}$ and $\mathcal{D}_{j}$, we denote their overlap by

$$
\begin{aligned}
\mathcal{O}_{j-1}=\frac{\partial^{3}}{\partial y_{1}^{3}} & -4\left(\sum_{2 \leq i \leq j-1}\left(\frac{1}{y_{i 1}^{2}}-\frac{1}{y_{i 1}} \frac{\partial}{\partial y_{i}}\right)+\sum_{2 j+1 \leq i \leq 4 N}\left(\frac{1 / 4}{\left(x_{i}-y_{1}\right)^{2}}-\frac{1}{\left(x_{i}-y_{1}\right)} \frac{\partial}{\partial x_{i}}\right)\right) \frac{\partial}{\partial y_{1}} \\
& +2\left(\sum_{2 \leq i \leq j-1}\left(\frac{2}{y_{i 1}^{3}}-\frac{1}{y_{i 1}^{2}} \frac{\partial}{\partial y_{i}}\right)+\sum_{2 j+1 \leq i \leq 4 N}\left(\frac{1 / 2}{\left(x_{i}-y_{1}\right)^{3}}-\frac{1}{\left(x_{i}-y_{1}\right)^{2}} \frac{\partial}{\partial x_{i}}\right)\right) .
\end{aligned}
$$

Then, we have

$$
\begin{aligned}
\mathcal{D}_{j-1}=\mathcal{O}_{j-1} & +\left(\frac{-1}{\left(x_{2 j-1}-y_{1}\right)^{2}}+\frac{4}{\left(x_{2 j-1}-y_{1}\right)} \frac{\partial}{\partial x_{2 j-1}}\right) \frac{\partial}{\partial y_{1}} \\
& +\left(\frac{-1}{\left(x_{2 j}-y_{1}\right)^{2}}+\frac{4}{\left(x_{2 j}-y_{1}\right)} \frac{\partial}{\partial x_{2 j}}\right) \frac{\partial}{\partial y_{1}} \\
& +\left(\frac{1}{\left(x_{2 j-1}-y_{1}\right)^{3}}+\frac{-2}{\left(x_{2 j-1}-y_{1}\right)^{2}} \frac{\partial}{\partial x_{2 j-1}}\right) \\
& +\left(\frac{1}{\left(x_{2 j}-y_{1}\right)^{3}}+\frac{-2}{\left(x_{2 j}-y_{1}\right)^{2}} \frac{\partial}{\partial x_{2 j}}\right) \\
\mathcal{D}_{j}=\mathcal{O}_{j-1} & +\left(\frac{-4}{y_{j 1}^{2}}+\frac{4}{y_{j 1}} \frac{\partial}{\partial y_{j}}\right) \frac{\partial}{\partial y_{1}}+\left(\frac{4}{y_{j 1}^{3}}+\frac{-2}{y_{j 1}^{2}} \frac{\partial}{\partial y_{j}}\right) .
\end{aligned}
$$

We set $G_{j-1}=\left(x_{2 j}-x_{2 j-1}\right)^{-1 / 2} F_{j-1}$. From $\mathcal{D}_{j-1} F_{j-1}=0$, we have

$$
\begin{aligned}
0= & \mathcal{O}_{j-1} G_{j-1}+\left(\frac{-1}{\left(x_{2 j-1}-y_{1}\right)^{2}}+\frac{-1}{\left(x_{2 j}-y_{1}\right)^{2}}+\frac{-2}{\left(x_{2 j-1}-y_{1}\right)\left(x_{2 j}-y_{1}\right)}\right) \frac{\partial}{\partial y_{1}} G_{j-1} \\
& +\left(\frac{1}{\left(x_{2 j-1}-y_{1}\right)^{3}}+\frac{1}{\left(x_{2 j}-y_{1}\right)^{3}}+\frac{\left(x_{2 j}-x_{2 j-1}\right)+2\left(x_{2 j-1}-y_{1}\right)}{\left(x_{2 j-1}-y_{1}\right)^{2}\left(x_{2 j}-y_{1}\right)^{2}}\right) G_{j-1} \\
& +\left(\frac{4}{\left(x_{2 j-1}-y_{1}\right)} \frac{\partial}{\partial y_{1}}+\frac{-2}{\left(x_{2 j-1}-y_{1}\right)^{2}}\right) \frac{\partial}{\partial x_{2 j-1}} G_{j-1} \\
& +\left(\frac{4}{\left(x_{2 j}-y_{1}\right)} \frac{\partial}{\partial y_{1}}+\frac{-2}{\left(x_{2 j}-y_{1}\right)^{2}}\right) \frac{\partial}{\partial x_{2 j}} G_{j-1} .
\end{aligned}
$$

We will argue that

$\mathcal{K} G_{j-1}\left(y_{1}, \ldots, y_{j-1}, x_{2 j-1}, \ldots, x_{4 N}\right) \rightarrow \mathcal{K} F_{j}\left(y_{1}, \ldots, y_{j}, x_{2 j+1}, \ldots, x_{4 N}\right), \quad$ as $x_{2 j-1}, x_{2 j} \rightarrow y_{j}$,

where

$\mathcal{K} \in\left\{1, \frac{\partial}{\partial y_{1}}, \frac{\partial^{3}}{\partial y_{1}^{3}}\right\} \cup\left\{\frac{\partial}{\partial y_{i}}, \frac{\partial^{2}}{\partial y_{i} \partial y_{1}}: 2 \leq i \leq j-1\right\} \cup\left\{\frac{\partial}{\partial x_{n}}, \frac{\partial^{2}}{\partial x_{n} \partial y_{1}}: 2 j+1 \leq n \leq 4 N\right\} ;$ and that

$$
\begin{aligned}
& \left(\frac{1}{\left(x_{2 j-1}-y_{1}\right)^{2}} \frac{\partial}{\partial x_{2 j-1}}+\frac{1}{\left(x_{2 j}-y_{1}\right)^{2}} \frac{\partial}{\partial x_{2 j}}\right) G_{j-1}\left(y_{1}, \ldots, y_{j-1}, x_{2 j-1}, \ldots, x_{4 N}\right) \\
& \rightarrow \frac{1}{y_{j 1}^{2}} \frac{\partial}{\partial y_{j}} F_{j}\left(y_{1}, \ldots, y_{j}, x_{2 j+1}, \ldots, x_{4 N}\right), \quad \text { as } x_{2 j-1}, x_{2 j} \rightarrow y_{j} \\
& \left(\frac{1}{\left(x_{2 j-1}-y_{1}\right)} \frac{\partial}{\partial x_{2 j-1}}+\frac{1}{\left(x_{2 j}-y_{1}\right)} \frac{\partial}{\partial x_{2 j}}\right) \frac{\partial}{\partial y_{1}} G_{j-1}\left(y_{1}, \ldots, y_{j-1}, x_{2 j-1}, \ldots, x_{4 N}\right) \\
& \rightarrow \frac{1}{y_{j 1}} \frac{\partial^{2}}{\partial y_{j} \partial y_{1}} F_{j}\left(y_{1}, \ldots, y_{j}, x_{2 j+1}, \ldots, x_{4 N}\right), \quad \text { as } x_{2 j-1}, x_{2 j} \rightarrow y_{j} .
\end{aligned}
$$




\section{Crossing probabilities in metric graph GFF}

From the proof of Proposition 5.6, Eq. (5.9) holds for $\mathcal{K}=1$. Furthermore, as $G_{j-1}$ is a finite linear combination of terms of the form

$$
\prod\left(x_{a}-x_{b}\right)^{ \pm 1 / 2} \times \prod\left(y_{k}-y_{l}\right)^{ \pm 2} \times \prod\left(x_{n}-y_{m}\right)^{ \pm 1},
$$

we view $G_{j-1}$ as a function on distinct complex variables $\left(y_{1}, \ldots, y_{j-1}, x_{2 j-1}, x_{2 j}, \ldots, x_{4 N}\right)$. We fix arbitrarily distinct complex points $\left(y_{1}, \ldots, y_{j-1}, x_{2 j+1}, \ldots, x_{4 N}\right)$ and denote $\boldsymbol{y}=$ $\left(y_{1}, \ldots, y_{j-1}\right)$ and $\boldsymbol{x}=\left(x_{2 j+1}, \ldots, x_{4 N}\right)$. Then $G_{j-1}$ is a meromorphic function of $\epsilon=$ $x_{2 j}-x_{2 j-1}$ and its Laurent series can be written as:

$$
\begin{aligned}
H_{j-1}\left(\boldsymbol{y}, x_{2 j-1}, \epsilon, \boldsymbol{x}\right): & =G_{j-1}\left(\boldsymbol{y}, x_{2 j-1}, x_{2 j-1}+\epsilon, \boldsymbol{x}\right) \\
& =F_{j}\left(\boldsymbol{y}, x_{2 j-1}, \boldsymbol{x}\right)+\sum_{n \geq 1} K_{n}\left(\boldsymbol{y}, x_{2 j-1}, \boldsymbol{x}\right) \epsilon^{n},
\end{aligned}
$$

where $K_{n}$ is a finite linear combination of terms of the form

$$
\prod\left(x_{a}-x_{b}\right)^{p / 2} \times \prod\left(y_{k}-y_{l}\right)^{ \pm 2} \times \prod\left(x_{n}-y_{m}\right)^{q}
$$

with $p, q \in \mathbb{Z}$.

Now, we prove (5.10). We have

$$
\begin{aligned}
& \left(\frac{1}{\left(x_{2 j-1}-y_{1}\right)^{2}} \frac{\partial}{\partial x_{2 j-1}}+\frac{1}{\left(x_{2 j}-y_{1}\right)^{2}} \frac{\partial}{\partial x_{2 j}}\right) G_{j-1}\left(\boldsymbol{y}, x_{2 j-1}, x_{2 j}, \boldsymbol{x}\right) \\
= & \left(\frac{1}{\left(x_{2 j-1}-y_{1}\right)^{2}} \frac{\partial}{\partial x_{2 j-1}}+\left(\frac{1}{\left(x_{2 j}-y_{1}\right)^{2}}-\frac{1}{\left(x_{2 j-1}-y_{1}\right)^{2}}\right) \frac{\partial}{\partial \epsilon}\right) H_{j-1}\left(\boldsymbol{y}, x_{2 j-1}, \epsilon, \boldsymbol{x}\right) .
\end{aligned}
$$

Thus, it suffices to prove, as $x_{2 j-1} \rightarrow y_{j}, \epsilon \rightarrow 0$,

$$
\frac{\partial}{\partial x_{2 j-1}} H_{j-1}\left(\boldsymbol{y}, x_{2 j-1}, \epsilon, \boldsymbol{x}\right) \rightarrow \frac{\partial}{\partial y_{j}} F_{j}\left(\boldsymbol{y}, y_{j}, \boldsymbol{x}\right), \quad \frac{\partial}{\partial \epsilon} H_{j-1}\left(\boldsymbol{y}, x_{2 j-1}, \epsilon, \boldsymbol{x}\right) \rightarrow K_{1}\left(\boldsymbol{y}, y_{j}, \boldsymbol{x}\right) .
$$

We define $\delta_{0}=\min \left\{\frac{x_{2 j+1}-y_{j}}{4}, \frac{y_{j}-y_{j-1}}{4}\right\}$ and $H_{j-1}\left(\boldsymbol{y}, x_{2 j-1}, 0, \boldsymbol{x}\right):=F_{j}\left(\boldsymbol{y}, x_{2 j-1}, \boldsymbol{x}\right)$. Then, the function $\left(x_{2 j-1}, \epsilon\right) \mapsto H_{j-1}\left(\boldsymbol{y}, x_{2 j-1}, \epsilon, \boldsymbol{x}\right)$ is continuous on $\left[y_{j}-\delta_{0}, y_{j}+\delta_{0}\right] \times$ $B\left(0, \delta_{0}\right)$ where $B\left(0, \delta_{0}\right):=\left\{z \in \mathbb{C}: d(z, 0)<\delta_{0}\right\}$. Moreover, for every $x_{2 j-1} \in\left[y_{j}-\right.$ $\left.\delta_{0}, y_{j}+\delta_{0}\right]$, the function $\epsilon \mapsto H_{j-1}\left(\boldsymbol{y}, x_{2 j-1}, \epsilon, \boldsymbol{x}\right)$ is holomorphic in $B\left(0, \delta_{0}\right) \backslash\{0\}$. Thus, the function $\epsilon \mapsto H_{j-1}\left(\boldsymbol{y}, x_{2 j-1}, \epsilon, \boldsymbol{x}\right)$ is holomorphic in $B\left(0, \delta_{0}\right)$. Then, we have

$$
K_{n}\left(\boldsymbol{y}, x_{2 j-1}, \boldsymbol{x}\right)=\frac{1}{2 \pi i} \int_{\partial B\left(0, \delta_{0}\right)} \frac{H_{j-1}\left(\boldsymbol{y}, x_{2 j-1}, z, \boldsymbol{x}\right)}{z^{n+1}} d z .
$$

Note that, there exists $M=M\left(\boldsymbol{y}, \boldsymbol{x}, y_{j}\right)>0$ such that, for all $x_{2 j-1} \in\left[y_{j}-\delta_{0}, y_{j}+\delta_{0}\right]$ and $z \in B\left(0,2 \delta_{0}\right) \backslash B\left(0, \frac{\delta_{0}}{2}\right)$,

$$
\left|H_{j-1}\left(\boldsymbol{y}, x_{2 j-1}, z, \boldsymbol{x}\right)\right| \leq M, \quad \text { and } \quad\left|\frac{\partial}{\partial x_{2 j-1}} H_{j-1}\left(\boldsymbol{y}, x_{2 j-1}, z, \boldsymbol{x}\right)\right| \leq M .
$$

Thus, we have

$$
\left|K_{n}\left(\boldsymbol{y}, x_{2 j-1}, \boldsymbol{x}\right)\right| \leq \frac{M}{\delta_{0}^{n}},
$$

and

$$
\left|\frac{\partial}{\partial x_{2 j-1}} K_{n}\left(\boldsymbol{y}, x_{2 j-1}, \boldsymbol{x}\right)\right|=\left|\frac{1}{2 \pi i} \int_{\partial B\left(0, \delta_{0}\right)} \frac{\frac{\partial}{\partial x_{2 j-1}} H_{j-1}\left(\boldsymbol{y}, x_{2 j-1}, z, \boldsymbol{x}\right)}{z^{n+1}} d z\right| \leq \frac{M}{\delta_{0}^{n}} .
$$


These imply that, for every $x_{2 j-1} \in\left[y_{j}-\delta_{0}, y_{j}+\delta_{0}\right]$ and $\epsilon \in B\left(0, \frac{\delta_{0}}{2}\right)$,

$$
\frac{\partial}{\partial x_{2 j-1}} H_{j-1}\left(\boldsymbol{y}, x_{2 j-1}, \epsilon, \boldsymbol{x}\right)=\frac{\partial}{\partial x_{2 j-1}} F_{j}\left(\boldsymbol{y}, x_{2 j-1}, \boldsymbol{x}\right)+\sum_{n \geq 1} \frac{\partial}{\partial x_{2 j-1}} K_{n}\left(\boldsymbol{y}, x_{2 j-1}, \boldsymbol{x}\right) \epsilon^{n},
$$

and

$$
\frac{\partial}{\partial \epsilon} H_{j-1}\left(\boldsymbol{y}, x_{2 j-1}, \epsilon, x_{2 j+1} \ldots, x_{4 N}\right)=\sum_{n \geq 1} n K_{n}\left(\boldsymbol{y}, x_{2 j-1}, \boldsymbol{x}\right) \epsilon^{n-1} .
$$

These give (5.12), and complete the proof of (5.10). Eq. (5.9) and (5.11) can be proved in a similar way.

Plugging (5.9)-(5.11) into (5.8), and letting $x_{2 j-1}, x_{2 j} \rightarrow y_{j}$, we obtain $\mathcal{D}_{j} F_{j}=0$. This completes the proof of (5.7). Taking $j=2 N$ in (5.7), we obtain the third order PDE (1.8) as desired. This completes the proof.

\subsection{Proof of Theorem 1.1}

In this section, we complete the proof of Theorem 1.1. Before that, we first address the coefficient $\mathcal{M}_{\omega, \tau(\hat{\alpha})}$ in the theorem.

Lemma 5.9. Fix $N \geq 1$ and the index valences $\varsigma=(2, \ldots, 2)$ of length $2 N$. Define $\omega \in \mathrm{DP}_{2 \mathrm{~N}}$ to be: $\forall j \in\{0,1, \ldots, N-1\}$,

$$
\omega(4 j)=0, \quad \omega(4 j+1)=1, \quad \omega(4 j+2)=2, \quad \omega(4 j+3)=1, \quad \omega(4 j+4)=0 .
$$

For any $\hat{\alpha} \in \mathrm{LP}_{\varsigma}$, let $\tau(\hat{\alpha}) \in \mathrm{PP}_{2 \mathrm{~N}}$ be the associated planar pair partition as defined in Section 2.2. Recall the definition of the incidence matrix $\mathcal{M}$ from (3.11). Then

$$
\mathcal{M}_{\omega, \beta}=1 \quad \text { implies } \quad \beta=\tau(\hat{\alpha}) \text { for some } \hat{\alpha} \in \mathrm{LP}_{\varsigma} .
$$

However, the converse does not hold in general.

Proof. Recall from Section 2.2 that $\tau$ introduces a bijection between $\mathrm{LP}_{\varsigma}$ and the collection $\left\{\beta \in \mathrm{PP}_{2 \mathrm{~N}}: \wedge_{2 \mathrm{j}-1} \notin \beta\right.$, for all $\left.1 \leq \mathrm{j} \leq 2 \mathrm{~N}\right\}$. Thus, it suffices to prove that $\wedge_{2 j-1} \notin \beta$ for every $1 \leq j \leq 2 N$. By definition,

$$
\omega=\{\{4 j+1,4 j+4\},\{4 j+2,4 j+3\}: 1 \leq j \leq N-1\} .
$$

Note that $\mathcal{M}_{\omega, \beta}=1$ implies there exists a $\sigma$ which is a permutation of $\{4 j+3,4 j+4$ : $0 \leq j \leq N-1\}$ such that

$$
\beta=\{\{4 j+1, \sigma(4 j+4)\},\{4 j+2, \sigma(4 j+3)\}: 0 \leq j \leq N-1\} .
$$

This implies $\wedge_{2 j-1} \notin \beta$ for every $1 \leq j \leq 2 N$.

Proof of Theorem 1.1 and (1.7). We use the same notations as in Section 5.3. By conformal invariance, we may assume $\Omega=\mathbb{H}$ and $y_{1}<\cdots<y_{2 N}$. Suppose $\Gamma$ is zero-boundary GFF on $\mathbb{H}$ and let $u$ be the harmonic function with the boundary data (1.5). From Proposition 5.3, we have

$$
\lim _{\delta \rightarrow 0} \mathbb{P}\left[\mathcal{A}^{\delta}=\hat{\alpha}\right]=\mathbb{P}[\mathcal{A}=\hat{\alpha}] .
$$

Let $\eta_{2 j-1}$ be the level line of the continuum GFF $\Gamma+u$ starting from $y_{2 j-1}$ with height $\lambda$ for $1 \leq j \leq N$; and let $\eta_{2 j}$ be the level line of $-(\Gamma+u)$ starting from $y_{2 j}$ with height $\lambda$ for $1 \leq j \leq N$. Note that the collection $\left\{\eta_{2}, \eta_{4}, \ldots, \eta_{2 N}\right\}$ coincides with the collection of level lines $\Gamma+u$ starting from $y_{2 j-1}$ with height $-\lambda$ for $1 \leq j \leq N$. See Figure 8. From 

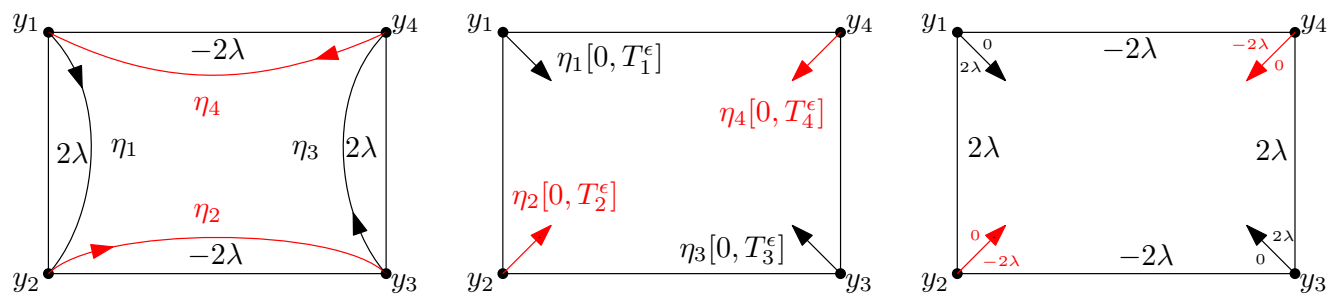

Figure 8: Consider continuum GFF $\Gamma+u$ in rectangle with alternating boundary data. In the left panel, we have four level lines: Let $\eta_{1}$ (resp. $\eta_{3}$ ) be the level line of $\Gamma+u$ starting from $y_{1}$ (resp. from $y_{3}$ ) with height $\lambda$. These two curves are in black.Let $\eta_{2}$ (resp. $\eta_{4}$ ) be the level line of $-(\Gamma+u)$ starting from $y_{2}$ (resp. from $y_{4}$ ) with height $\lambda$. These two curves are in red. The middle panel indicates the domain $H_{\epsilon}$ which is obtained by removing from $\mathbb{H}$ the four pieces $\eta_{i}\left[0, T_{i}^{\epsilon}\right]$ with $i=1,2,3,4$. In the right panel, we see that the boundary data of $\Gamma+u$ in $H_{\epsilon}$ is piecewise constant: $0,2 \lambda, 0,-2 \lambda, 0,2 \lambda, 0,-2 \lambda$.

Lemma 5.4, the frontier of $\mathbb{A}$ and of $\mathbb{V}$ has the same law as $\cup_{1 \leq j \leq 2 N} \eta_{j}$. It suffices to prove

$$
\mathbb{P}\left[\left\{\eta_{1}, \ldots, \eta_{2 N}\right\} \text { forms the planar link pattern } \hat{\alpha}\right]=\mathcal{M}_{\omega, \tau(\hat{\alpha})} \frac{\mathcal{Z}_{\hat{\alpha}}\left(y_{1}, \ldots, y_{2 N}\right)}{\mathcal{Z}_{\mathrm{mGFF}}^{(N)}\left(y_{1}, \ldots, y_{2 N}\right)},
$$

where $\omega$ and $\mathcal{M}_{\omega, \tau(\hat{\alpha})}$ are defined in Lemma 5.9.

For $1 \leq j \leq 2 N$ and $\epsilon>0$ small, we denote $T_{j}^{\epsilon}=\inf \left\{t>0: d\left(\eta_{j}(t), y_{j}\right)=\epsilon\right\}$. We take $\phi^{\epsilon}$ to be the conformal map from

$$
H_{\epsilon}:=\mathbb{H} \backslash\left(\cup_{1 \leq j \leq 2 N} \eta_{j}\left[0, T_{j}^{\epsilon}\right]\right)
$$

onto $\mathbb{H}$ normalized at $\infty$. Then, we see that, given $H_{\epsilon}$, the event

$$
\left\{\left\{\eta_{1}, \ldots, \eta_{2 N}\right\} \text { forms the planar link pattern } \hat{\alpha}\right\}
$$

is the same as

$$
\left\{\left\{\phi^{\epsilon}\left(\eta_{1}\right), \ldots, \phi^{\epsilon}\left(\eta_{2 N}\right)\right\} \text { forms the planar link pattern } \tau(\hat{\alpha})\right\}
$$

where $\tau(\hat{\alpha})$ is defined in Section 2.2.

Now, let us consider the collection $\left\{\phi^{\epsilon}\left(\eta_{1}\right), \ldots, \phi^{\epsilon}\left(\eta_{2 N}\right)\right\}$. The conditional law of $\Gamma+u$ given $H_{\epsilon}$ is a GFF in $H_{\epsilon}$ with the following boundary data: for $1 \leq j \leq 2 N$,

$$
2 \lambda \text { on }\left(y_{2 j-1}^{+}, y_{2 j}^{-}\right), \quad 0 \text { along the left side of } \eta_{2 j}\left[0, T_{2 j}^{\epsilon}\right] \text {, }
$$

$-2 \lambda$ along the right side of $\eta_{2 j}\left[0, T_{2 j}^{\epsilon}\right]$,

$-2 \lambda$ on $\left(y_{2 j}^{+}, y_{2 j+1}^{-}\right), \quad 0$ along the left side of $\eta_{2 j+1}\left[0, T_{2 j+1}^{\epsilon}\right]$,

$2 \lambda$ along the right side of $\eta_{2 j+1}\left[0, T_{2 j+1}^{\epsilon}\right]$.

See Figure 8. Then, we have

$\mathbb{P}\left[\left\{\eta_{1}, \ldots, \eta_{2 N}\right\}\right.$ forms the planar link pattern $\left.\hat{\alpha}\right]$

$=\mathbb{E}\left[\mathbb{P}\left[\left\{\eta_{1}, \ldots, \eta_{2 N}\right\}\right.\right.$ forms the planar link pattern $\left.\left.\hat{\alpha} \mid H_{\epsilon}\right]\right]$

$=\mathbb{E}\left[\mathbb{P}\left[\left\{\phi^{\epsilon}\left(\eta_{1}\right), \ldots, \phi^{\epsilon}\left(\eta_{2 N}\right)\right\}\right.\right.$ forms the planar link pattern $\left.\left.\tau(\hat{\alpha}) \mid H_{\epsilon}\right]\right]$

$=\mathbb{E}\left[\mathcal{M}_{\omega, \tau(\hat{\alpha})} \frac{\mathcal{Z}_{\tau(\hat{\alpha})}\left(\phi^{\epsilon}\left(y_{1}^{-}\right), \phi^{\epsilon}\left(\eta_{1}\left(T_{1}^{\epsilon}\right)\right), \phi^{\epsilon}\left(y_{2}^{-}\right), \phi^{\epsilon}\left(\eta_{2}\left(T_{2}^{\epsilon}\right)\right), \ldots, \phi^{\epsilon}\left(y_{2 N}^{+}\right), \phi^{\epsilon}\left(\eta_{2 N}\left(T_{2 N}^{\epsilon}\right)\right)\right)}{\mathcal{U}_{\omega}\left(\phi^{\epsilon}\left(y_{1}^{-}\right), \phi^{\epsilon}\left(\eta_{1}\left(T_{1}^{\epsilon}\right)\right), \phi^{\epsilon}\left(y_{2}^{-}\right), \phi^{\epsilon}\left(\eta_{2}\left(T_{2}^{\epsilon}\right)\right), \ldots, \phi^{\epsilon}\left(y_{2 N}^{+}\right), \phi^{\epsilon}\left(\eta_{2 N}\left(T_{2 N}^{\epsilon}\right)\right)\right)}\right]$

$=\mathcal{M}_{\omega, \tau(\hat{\alpha})} \frac{\mathcal{Z}_{\hat{\alpha}}\left(y_{1}, \ldots, y_{2 N}\right)}{\mathcal{U}_{(\omega)_{2}}^{4}\left(y_{1}, \ldots, y_{2 N}\right)}$, 
where $(\omega)_{2}$ is defined as in Lemma 5.7. In the second last equal sign, we use Theorem 4.1: consider the GFF in $H_{\epsilon}$, the collection $\left\{\phi^{\epsilon}\left(\eta_{2}\right), \phi^{\epsilon}\left(\eta_{4}\right), \ldots, \phi^{\epsilon}\left(\eta_{2 N}\right)\right\}$ coincides with the collection of level lines starting from $y_{2 j-1}$ with height $-\lambda$. Therefore, the connection probability is given by $\mathcal{M}_{\omega, \tau(\hat{\alpha})} \mathcal{Z}_{\tau(\hat{\alpha})} / \mathcal{U}_{\omega}$. In the last equal sign, we let $\epsilon \rightarrow 0$. Combining Proposition 5.6, Lemma 5.7, and dominated convergence theorem, we obtain the conclusion.

Finally, from the above analysis, we have

$$
\lim _{\delta \rightarrow 0} \mathbb{P}\left[\mathcal{A}^{\delta}=\hat{\alpha}\right]=\mathbb{P}[\mathcal{A}=\hat{\alpha}]=\mathcal{M}_{\omega, \tau(\hat{\alpha})} \frac{\mathcal{Z}_{\hat{\alpha}}\left(y_{1}, \ldots, y_{2 N}\right)}{\mathcal{U}_{(\omega)_{2}}^{4}\left(y_{1}, \ldots, y_{2 N}\right)}, \quad \text { for all } \hat{\alpha} \in \mathrm{LP}_{\varsigma} .
$$

Furthermore, from (3.12) and (5.13), we have

$$
\sum_{\hat{\alpha} \in \mathrm{LP}_{\varsigma}} \mathcal{M}_{\omega, \tau(\hat{\alpha})} \frac{\mathcal{Z}_{\hat{\alpha}}\left(y_{1}, \ldots, y_{2 N}\right)}{\mathcal{U}_{(\omega)_{2}}^{4}\left(y_{1}, \ldots, y_{2 N}\right)}=1 .
$$

Thus

$$
\mathcal{Z}_{\mathrm{mGFF}}^{(N)}\left(y_{1}, \ldots, y_{2 N}\right):=\sum_{\hat{\alpha} \in \mathrm{LP}_{\varsigma}} \mathcal{M}_{\omega, \tau(\hat{\alpha})} \mathcal{Z}_{\hat{\alpha}}\left(y_{1}, \ldots, y_{2 N}\right)=\mathcal{U}_{(\omega)_{2}}^{4}\left(y_{1}, \ldots, y_{2 N}\right) .
$$

This completes the proof of (1.7).
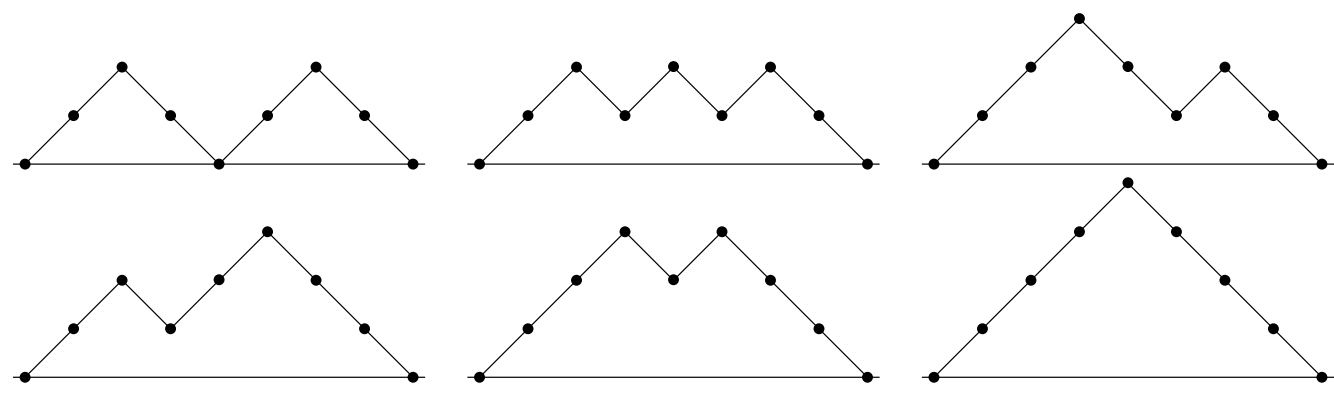

Figure 9: There are six Dyck paths in this figure: in the first row, from left to right, we denote them by $\alpha_{1}, \alpha_{2}, \alpha_{3}$ respectively; in the second row, from left to right, we denote them by $\alpha_{4}, \alpha_{5}, \alpha_{6}$ respectively. We see that $\alpha_{1} \preceq \alpha_{2} \preceq \alpha_{3}, \alpha_{4} \preceq \alpha_{5} \preceq \alpha_{6}$.

Corollary 5.10. The conclusion in (1.3) holds.

Proof. We define $\beta_{1}, \beta_{2}, \beta_{3}$ as in Figure 5 and we define $\alpha_{1}, \ldots, \alpha_{6}$ as in Figure 9. From (3.12), we have

$$
\begin{aligned}
& \mathcal{Z}_{\beta_{1}}=\mathcal{U}_{\alpha_{2}}-\mathcal{U}_{\alpha_{3}}-\mathcal{U}_{\alpha_{4}}+\mathcal{U}_{\alpha_{5}}-2 \mathcal{U}_{\alpha_{6}}, \\
& \mathcal{Z}_{\beta_{2}}=\mathcal{U}_{\alpha_{6}}, \\
& \mathcal{Z}_{\beta_{3}}=\mathcal{U}_{\alpha_{1}}-\mathcal{U}_{\alpha_{2}}+\mathcal{U}_{\alpha_{3}}+\mathcal{U}_{\alpha_{4}}-\mathcal{U}_{\alpha_{5}}+\mathcal{U}_{\alpha_{6}} .
\end{aligned}
$$

Suppose $y_{1}<y_{2}<y_{3}<y_{4}$ and we need to derive the limits as

$$
x_{1}, x_{2} \rightarrow y_{1} ; \quad x_{3}, x_{4} \rightarrow y_{2} ; \quad x_{5}, x_{6} \rightarrow y_{3} ; \quad x_{7}, x_{8} \rightarrow y_{4} .
$$

We denote $x_{j i}=x_{j}-x_{i}$ for $1 \leq i<j \leq 8$ and $y_{j i}=y_{j}-y_{i}$ for $1 \leq i<j \leq 4$. Furthermore, we denote the cross-ratio by $q=\left(y_{21} y_{43}\right) /\left(y_{31} y_{42}\right)$. 
First, for $\alpha_{1}$ and $\alpha_{6}$, we have

$$
\begin{aligned}
\lim _{\substack{x_{1}, x_{2} \rightarrow y_{1} ; x_{3}, x_{4} \rightarrow y_{2} ; \\
x_{5}, x_{6} \rightarrow y_{3} ; x_{7}, x_{8} \rightarrow y_{4}}} \frac{\mathcal{U}_{\alpha_{1}}\left(x_{1}, \ldots, x_{8}\right)}{\sqrt{x_{21} x_{43} x_{65} x_{87}}}=\left(\frac{y_{31} y_{42}}{y_{21} y_{41} y_{32} y_{43}}\right)^{2}=\frac{1}{q^{2}(1-q)^{2} y_{31}^{2} y_{42}^{2}}, \\
\lim _{\substack{x_{1}, x_{2} \rightarrow y_{1} ; x_{3}, x_{4} \rightarrow y_{2} ; \\
x_{5}, x_{6} \rightarrow y_{3} ; x_{7}, x_{8} \rightarrow y_{4}}} \frac{\mathcal{U}_{\alpha_{6}}\left(x_{1}, \ldots, x_{8}\right)}{\sqrt{x_{21} x_{43} x_{65} x_{87}}}=\left(\frac{y_{21} y_{43}}{y_{31} y_{41} y_{32} y_{42}}\right)^{2}=\frac{q^{2}}{(1-q)^{2} y_{31}^{2} y_{42}^{2}} .
\end{aligned}
$$

Second, for $n=2,3,4,5$, we have

$$
\lim _{\substack{x_{1}, x_{2} \rightarrow y_{1} ; \\ x_{7}, x_{8} \rightarrow y_{4}}} \frac{\mathcal{U}_{\alpha_{n}}\left(x_{1}, \ldots, x_{8}\right)}{\sqrt{x_{21} x_{87}}}=y_{41}^{-2} \times \prod_{3 \leq i \leq 6}\left(x_{i}-y_{1}\right)^{\vartheta_{\alpha_{n}}(i, 1)}\left(y_{4}-x_{i}\right)^{\vartheta_{\alpha_{n}}(i, 7)} \times \prod_{3 \leq i<j \leq 6} x_{j i}^{\frac{1}{2} \vartheta_{\alpha_{n}}(i, j)} .
$$

Taking the difference between $\mathcal{U}_{\alpha_{2}}$ and $\mathcal{U}_{\alpha_{3}}$ and the difference between $\mathcal{U}_{\alpha_{4}}$ and $\mathcal{U}_{\alpha_{5}}$, we have

$$
\begin{aligned}
& \lim _{\substack{x_{1}, x_{2} \rightarrow y_{1}, x_{3}, x_{4} \rightarrow y_{2} ; \\
x_{7}, x_{8} \rightarrow y_{4}}} \frac{\left(\mathcal{U}_{\alpha_{2}}-\mathcal{U}_{\alpha_{3}}\right)\left(x_{1}, \ldots, x_{8}\right)}{\sqrt{x_{21} x_{43} x_{87}}} \\
= & y_{41}^{-2} \times \frac{\left(x_{6}-y_{1}\right)}{\left(x_{5}-y_{1}\right)} \frac{\left(y_{4}-x_{5}\right)}{\left(y_{4}-x_{6}\right)} \times\left(\frac{\sqrt{x_{65}}}{\left(x_{5}-y_{2}\right)\left(x_{6}-y_{2}\right)}+\frac{2 y_{41}}{y_{21} y_{42} \sqrt{x_{65}}}\right), \\
& \lim _{x_{1}, x_{2} \rightarrow y_{1}, x_{3}, x_{4} \rightarrow y_{2} ;} \frac{\left(\mathcal{U}_{\alpha_{4}}-\mathcal{U}_{\alpha_{5}}\right)\left(x_{1}, \ldots, x_{8}\right)}{\sqrt{x_{21} x_{43} x_{87}}} \\
= & y_{41}^{-2} \times \frac{\left(x_{5}-y_{1}\right)\left(y_{4}-x_{6}\right)}{\left(x_{6}-y_{1}\right)\left(y_{4}-x_{5}\right)} \times\left(\frac{-\sqrt{x_{65}}}{\left(x_{5}-y_{2}\right)\left(x_{6}-y_{2}\right)}+\frac{2 y_{41}}{y_{21} y_{42} \sqrt{x_{65}}}\right) .
\end{aligned}
$$

Taking the difference between these two, we have

$$
\begin{aligned}
& \lim _{\substack{x_{1}, x_{2} \rightarrow y_{1} ; x_{3}, x_{4} \rightarrow y_{2} ; \\
x_{5}, x_{6} \rightarrow y_{3} ; x_{7}, x_{8} \rightarrow y_{4}}} \frac{\left(\mathcal{U}_{\alpha_{2}}-\mathcal{U}_{\alpha_{3}}-\mathcal{U}_{\alpha_{4}}+\mathcal{U}_{\alpha_{5}}\right)\left(x_{1}, \ldots, x_{8}\right)}{\sqrt{x_{21} x_{43} x_{65} x_{87}}} \\
= & \frac{2}{y_{41}^{2} y_{32}^{2}}+\frac{4}{y_{21} y_{31} y_{42} y_{43}}=\left(\frac{2}{(1-q)^{2}}+\frac{4}{q}\right) \frac{1}{y_{31}^{2} y_{42}^{2}} .
\end{aligned}
$$

Plugging (5.15), (5.16) and (5.17) into (5.14), we have

$$
\begin{aligned}
& \lim _{\substack{x_{1}, x_{2} \rightarrow y_{1} ; x_{3}, x_{4} \rightarrow y_{2} ; \\
x_{5}, x_{6} \rightarrow y_{3} ; x_{7}, x_{8} \rightarrow y_{4}}} \frac{\mathcal{Z}_{\beta_{1}}\left(x_{1}, \ldots, x_{8}\right)}{\mathcal{U}_{\alpha_{1}}\left(x_{1}, \ldots, x_{8}\right)}=2 q(1-q)\left(2-q+q^{2}\right), \\
& \lim _{\substack{x_{1}, x_{2} \rightarrow y_{1} ; x_{3}, x_{4} \rightarrow y_{2} ; \\
x_{5}, x_{6} \rightarrow y_{3} ; x_{7}, x_{8} \rightarrow y_{4}}} \underset{\mathcal{Z}_{\alpha_{2}}\left(x_{1}, \ldots, x_{8}\right)}{\left.\mathcal{U}_{1}, \ldots, x_{8}\right)}=q^{4}, \\
& \lim _{\substack{x_{1}, x_{2} \rightarrow y_{1} ; x_{3}, x_{4} \rightarrow y_{2} ; \\
x_{5}, x_{6} \rightarrow y_{3} ; x_{7}, x_{8} \rightarrow y_{4}}} \frac{\mathcal{Z}_{\beta_{3}}\left(x_{1}, \ldots, x_{8}\right)}{\mathcal{U}_{\alpha_{1}}\left(x_{1}, \ldots, x_{8}\right)}=(1-q)^{4} .
\end{aligned}
$$

The scaling limit of the crossing probability in (1.3) corresponds to the limit of $\mathcal{Z}_{\beta_{2}} / \mathcal{U}_{\alpha_{1}}$, see Figure 1 and Figure 5. This completes the proof.

\section{A Technical lemmas}

The following three lemmas are technical. Lemmas A.1 and A.2 are needed in the proof of Lemma A.3 which is essential in the proof of Theorem 4.1.

Lemma A.1. Let $x_{1}<x_{2}<x_{3}<x_{4}$. Suppose $\eta$ is a continuous simple curve in $\mathbb{H}$ starting from $x_{1}$ and terminating at $x_{4}$ at time $T$. Assume $\eta$ hits $\mathbb{R}$ only at its two end points. Let $\left(W_{t}, 0 \leq t \leq T\right)$ be its driving function and $\left(g_{t}, 0 \leq t \leq T\right)$ be the corresponding family of conformal maps. Then

$$
\lim _{t \rightarrow T} \frac{\left(g_{t}\left(x_{3}\right)-g_{t}\left(x_{2}\right)\right)\left(g_{t}\left(x_{4}\right)-W_{t}\right)}{\left(g_{t}\left(x_{3}\right)-W_{t}\right)\left(g_{t}\left(x_{4}\right)-g_{t}\left(x_{2}\right)\right)}=0 .
$$




\section{Crossing probabilities in metric graph GFF}

Proof. See [PW19, Lemma B.2].

Lemma A.2. Let $x_{0}<x_{1}<x_{2}<x_{3}<x_{4}$. Suppose $\eta$ is a continuous simple curve in $\mathbb{H}$ starting from $x_{0}$ and terminating at $x_{4}$ at time $T$. Assume $\eta$ hits $\mathbb{R}$ only at its two end points. Let $\left(W_{t}, 0 \leq t \leq T\right)$ be its driving function and $\left(g_{t}, 0 \leq t \leq T\right)$ be the corresponding family of conformal maps. Then there exist $C_{1}, C_{2}>0$, which depend on $\eta[0, T]$, such that for all $t \in[0, T]$,

$$
C_{1} \leq\left|\frac{\left(g_{t}\left(x_{2}\right)-g_{t}\left(x_{1}\right)\right)\left(g_{t}\left(x_{3}\right)-W_{t}\right)}{\left(g_{t}\left(x_{2}\right)-W_{t}\right)\left(g_{t}\left(x_{3}\right)-g_{t}\left(x_{1}\right)\right)}\right| \leq C_{2} .
$$

Proof. To prove the conclusion, we will show the following two estimates: First, we will show that there exist $C_{1}, C_{2}>0$, which only depend on $\eta[0, T]$, such that for all $t \in[0, T]$,

$$
C_{1} \leq \frac{g_{t}\left(x_{2}\right)-g_{t}\left(x_{1}\right)}{g_{t}\left(x_{3}\right)-g_{t}\left(x_{1}\right)} \leq C_{2} .
$$

Second, we will show

$$
\lim _{t \rightarrow T} \frac{g_{t}\left(x_{3}\right)-W_{t}}{g_{t}\left(x_{2}\right)-W_{t}}=1+\lim _{t \rightarrow T} \frac{g_{t}\left(x_{3}\right)-g_{t}\left(x_{2}\right)}{g_{t}\left(x_{2}\right)-W_{t}}=1 .
$$

In this proof, we use $\asymp$ to simplify notations: for two functions $f$ and $g$, the notation $f \asymp g$ means that there exists a constant $C>0$ which only depends on $\eta[0, T]$ such that $C^{-1} \leq f / g \leq C$.

We first show (A.1). Note that for an interval $[a, b]$, we have

$$
b-a=\lim _{y \rightarrow \infty} \pi y \mathbb{P}^{i y}[\mathrm{BM} \text { hits } \partial \mathbb{H} \text { in }[a, b]],
$$

where BM is the Brownian motion starts from iy. By conformal invariance of the Brownian motion, we have

$$
b-a=\lim _{y \rightarrow \infty} \pi y \mathbb{P}^{g_{t}^{-1}(i y)}\left[\mathrm{BM} \text { hits } \partial(\mathbb{H} \backslash \eta[0, t]) \text { in } g_{t}^{-1}([a, b])\right] .
$$

We choose $\delta_{0}$ small enough, such that the $\delta_{0}$-neighborhood of the interval $\left[x_{1}, x_{3}\right]$ does not intersect $\eta[0, T]$. We denote the boundary of this neighborhood in $\mathbb{H}$ by $\gamma$, this is a simple curve. For the Brownian motion starting from $g_{t}^{-1}(i y)$, let $\tau$ be the first time the Brownian motion hits $\gamma$. Consider the connected component $V$ of $\mathbb{H} \backslash \eta[0, T]$ which contains $x_{1}$ on its boundary and choose a point $z \in V$. Suppose $\mathbb{U}$ is the unit disk, and $\phi_{t}: \mathbb{H} \backslash \eta[0, t] \rightarrow \mathbb{U}$ is the conformal map with $\phi_{t}(z)=0, \phi_{t}^{\prime}(z)>0$. Suppose $\phi_{T}: V \rightarrow \mathbb{U}$ is the conformal map with the same normalization. Then, for any compact set $K \subset \bar{V}$ which does not intersect $\eta[0, T]$, the conformal map $\phi_{t}$ converges to $\phi_{T}$ uniformly on $K$.

Note that

$$
\begin{aligned}
& \mathbb{P}^{g_{t}^{-1}(i y)}\left[\mathrm{BM} \text { hits } \partial(\mathbb{H} \backslash \eta[0, t]) \text { in }\left[x_{1}, x_{2}\right]\right] \\
= & \mathbb{P}^{g_{t}^{-1}(i y)}\left[\mathbb{1}_{\{\tau<\infty\}} \mathbb{P}^{B_{\tau}}\left[\mathrm{BM} \text { hits } \partial(\mathbb{H} \backslash \eta[0, t]) \text { in }\left[x_{1}, x_{2}\right]\right]\right] .
\end{aligned}
$$

We will compare

$$
\mathbb{P}^{B_{\tau}}\left[\mathrm{BM} \text { hits } \partial(\mathbb{H} \backslash \eta[0, t]) \text { in }\left[x_{1}, x_{2}\right]\right] \quad \text { and } \quad \mathbb{P}^{B_{\tau}}\left[\text { BM hits } \partial(\mathbb{H} \backslash \eta[0, t]) \text { in }\left[x_{1}, x_{3}\right]\right] .
$$

In fact we can replace $B_{\tau}$ by a deterministic point on $\gamma$. For every $w \in \gamma$, we have

$$
\mathbb{P}^{w}\left[\mathrm{BM} \text { hits } \partial(\mathbb{H} \backslash \eta[0, t]) \text { in }\left[x_{1}, x_{2}\right]\right]=\mathbb{P}^{\phi_{t}(w)}\left[\mathrm{BM} \text { hits } \partial \mathbb{U} \text { in }\left[\phi_{t}\left(x_{1}\right), \phi_{t}\left(x_{2}\right)\right]\right],
$$




\section{Crossing probabilities in metric graph GFF}

where $\left[\phi_{t}\left(x_{1}\right), \phi_{t}\left(x_{2}\right)\right]$ is the conformal image of $\left[x_{1}, x_{2}\right]$. By direct computation, the right hand-side equals

$$
\frac{1}{2 \pi}\left(\arg \frac{\phi_{t}\left(x_{2}\right)-\phi_{t}(w)}{1-\overline{\phi_{t}(w)} \phi_{t}\left(x_{2}\right)}-\arg \frac{\phi_{t}\left(x_{1}\right)-\phi_{t}(w)}{1-\overline{\phi_{t}(w)} \phi_{t}\left(x_{1}\right)}\right),
$$

where arg is the argument principal which takes value in $[0,2 \pi)$. Note that there exists $\epsilon_{0}>0$ such that

$$
\frac{1}{2 \pi}\left(\arg \frac{\phi_{t}\left(x_{2}\right)-\phi_{t}(w)}{1-\overline{\phi_{t}(w)} \phi_{t}\left(x_{2}\right)}-\arg \frac{\phi_{t}\left(x_{1}\right)-\phi_{t}(w)}{1-\overline{\phi_{t}(w)} \phi_{t}\left(x_{1}\right)}\right) \leq 1-\epsilon_{0}
$$

because $\gamma$ is bounded away from $\left[x_{1}, x_{3}\right]$. Thus,

$$
\begin{aligned}
\mathbb{P}^{\phi_{t}(w)}\left[\mathrm{BM} \text { hits } \partial \mathbb{U} \text { in }\left[\phi_{t}\left(x_{1}\right), \phi_{t}\left(x_{2}\right)\right]\right] & \asymp\left|\frac{\phi_{t}\left(x_{2}\right)-\phi_{t}(w)}{1-\overline{\phi_{t}(w)} \phi_{t}\left(x_{2}\right)}-\frac{\phi_{t}\left(x_{1}\right)-\phi_{t}(w)}{1-\overline{\phi_{t}(w)} \phi_{t}\left(x_{1}\right)}\right| \\
& =\frac{\left(1-\left|\phi_{t}(w)\right|^{2}\right)\left|\phi_{t}\left(x_{2}\right)-\phi_{t}\left(x_{1}\right)\right|}{\left|1-\overline{\phi_{t}(w)} \phi_{t}\left(x_{2}\right)\right|\left|1-\overline{\phi_{t}(w)} \phi_{t}\left(x_{1}\right)\right|} .
\end{aligned}
$$

Similarly, we have

$$
\mathbb{P}^{\phi_{t}(w)}\left[\mathrm{BM} \text { hits } \partial \mathbb{U} \text { in }\left[\phi_{t}\left(x_{1}\right), \phi_{t}\left(x_{3}\right)\right]\right] \asymp \frac{\left(1-\left|\phi_{t}(w)\right|^{2}\right)\left|\phi_{t}\left(x_{3}\right)-\phi_{t}\left(x_{1}\right)\right|}{\left|1-\overline{\phi_{t}(w)} \phi_{t}\left(x_{3}\right)\right|\left|1-\overline{\phi_{t}(w)} \phi_{t}\left(x_{1}\right)\right|} .
$$

Therefore,

$$
\frac{\mathbb{P}^{\phi_{t}(w)}\left[\mathrm{BM} \text { hits } \partial \mathbb{U} \text { in }\left[\phi_{t}\left(x_{1}\right), \phi_{t}\left(x_{2}\right)\right]\right]}{\mathbb{P}^{\phi_{t}(w)}\left[\operatorname{BM} \text { hits } \partial \mathbb{U} \text { in }\left[\phi_{t}\left(x_{1}\right), \phi_{t}\left(x_{3}\right)\right]\right]} \asymp \frac{\left|\phi_{t}\left(x_{2}\right)-\phi_{t}\left(x_{1}\right)\right|}{\left|\phi_{t}\left(x_{3}\right)-\phi_{t}\left(x_{1}\right)\right|} \frac{\left|\phi_{t}\left(x_{3}\right)-\phi_{t}(w)\right|}{\left|\phi_{t}\left(x_{2}\right)-\phi_{t}(w)\right|} \asymp 1 .
$$

The last $\asymp$ is because of the uniform convergence of $\phi_{t}$. Thus, we have

$$
\mathbb{P}^{B_{\tau}}\left[\mathrm{BM} \text { hits } \partial(\mathbb{H} \backslash \eta[0, t]) \text { in }\left[x_{1}, x_{2}\right]\right] \asymp \mathbb{P}^{B_{\tau}}\left[\mathrm{BM} \text { hits } \partial(\mathbb{H} \backslash \eta[0, t]) \text { in }\left[x_{1}, x_{3}\right]\right] \text {. }
$$

This implies (A.1).

Next, we show (A.2). Consider the Brownian motion starting from $g_{t}^{-1}(i y)$. We define $C\left(x_{4}, \delta\right):=\left\{z \in \mathbb{H}: d\left(z, x_{4}\right)=\delta\right\}$. Let $\tau_{\delta}$ be the first time that it hits the connected component of half circle $C\left(x_{4}, \delta\right) \cap \mathbb{H} \backslash \eta[0, t]$ which contains $x_{4}-\delta$ on its boundary and we denote this connected component by $C_{\delta}$. Then we have

$$
\begin{aligned}
& \mathbb{P}^{g_{t}^{-1}(i y)}\left[\mathrm{BM} \text { hits } \partial(\mathbb{H} \backslash \eta[0, t]) \text { in the right side of } \eta[0, t] \cup\left[x_{0}, x_{2}\right]\right] \\
\geq & \mathbb{P}^{g_{t}^{-1}(i y)}\left[\mathbb{1}_{\left\{\tau_{\delta}<\infty\right\}} \mathbb{P}^{B_{\tau_{\delta}}}\left[\mathrm{BM} \text { hits } \partial(\mathbb{H} \backslash \eta[0, t]) \text { in the right side of } \eta[0, t] \cup\left[x_{0}, x_{2}\right]\right]\right],
\end{aligned}
$$

and

$$
\begin{aligned}
& \mathbb{P}^{g_{t}^{-1}(i y)}\left[\mathrm{BM} \text { hits } \partial(\mathbb{H} \backslash \eta[0, t]) \text { in }\left[x_{2}, x_{3}\right]\right] \\
= & \mathbb{P}^{g_{t}^{-1}(i y)}\left[\mathbb{1}_{\left\{\tau_{\delta}<\infty\right\}} \mathbb{P}^{B_{\tau_{\delta}}}\left[\mathrm{BM} \text { hits } \partial(\mathbb{H} \backslash \eta[0, t]) \text { in }\left[x_{2}, x_{3}\right]\right]\right] .
\end{aligned}
$$

By conformal invariance of the Brownian motion, we have

$$
\begin{aligned}
& \mathbb{P}^{B_{\tau_{\delta}}}\left[\mathrm{BM} \text { hits } \partial(\mathbb{H} \backslash \eta[0, t]) \text { in the right side of } \eta[0, t] \cup\left[x_{0}, x_{2}\right]\right] \\
= & \mathbb{P}^{\phi_{t}\left(B_{\tau_{\delta}}\right)}\left[\mathrm{BM} \text { hits } \partial \mathbb{U} \text { in }\left[\phi_{t}(\eta(t)), \phi_{t}\left(x_{2}\right)\right]\right],
\end{aligned}
$$




\section{Crossing probabilities in metric graph GFF}

where $\left[\phi_{t}(\eta(t)), \phi_{t}\left(x_{2}\right)\right]$ is the conformal image of the right side of $\eta[0, t] \cup\left[x_{0}, x_{2}\right]$. Moreover

$$
\begin{aligned}
& \mathbb{P}^{B_{\tau_{\delta}}}\left[\mathrm{BM} \text { hits } \partial(\mathbb{H} \backslash \eta[0, t]) \text { in }\left[x_{2}, x_{3}\right]\right] \\
= & \mathbb{P}^{\phi_{t}\left(B_{\tau_{\delta}}\right)}\left[\mathrm{BM} \text { hits } \partial \mathbb{U} \text { in }\left[\phi_{t}\left(x_{2}\right), \phi_{t}\left(x_{3}\right)\right]\right],
\end{aligned}
$$

where $\left[\phi_{t}\left(x_{2}\right), \phi_{t}\left(x_{3}\right)\right]$ is the conformal image of $\left[x_{2}, x_{3}\right]$. We replace $B_{\tau_{\delta}}$ by a deterministic point on $C_{\delta}$ for the same reason as in the proof of (A.1). For every $w \in C_{\delta}$, by Beurling estimate and conformal invariance, there exists $C>0$ such that

$$
\mathbb{P}^{\phi_{t}(w)}\left[\mathrm{BM} \text { hits } \partial \mathbb{U} \text { in }\left[\phi_{t}\left(x_{2}\right), \phi_{t}\left(x_{3}\right)\right]\right] \leq C\left(\frac{\delta}{x_{4}-x_{3}}\right)^{\frac{1}{2}} .
$$

This implies that there exists $\epsilon_{0}>0$ such that

$$
\mathbb{P}^{\phi_{t}(w)}\left[\mathrm{BM} \text { hits } \partial \mathbb{U} \text { in }\left[\phi_{t}\left(x_{2}\right), \phi_{t}\left(x_{3}\right)\right]\right] \leq 1-\epsilon_{0} .
$$

Thus, by the same method as in the proof of (A.1), we have

$$
\mathbb{P}^{\phi_{t}(w)}\left[\mathrm{BM} \text { hits } \partial \mathbb{U} \text { in }\left[\phi_{t}\left(x_{2}\right), \phi_{t}\left(x_{3}\right)\right]\right] \asymp \frac{\left(1-\left|\phi_{t}(w)\right|^{2}\right)\left|\phi_{t}\left(x_{3}\right)-\phi_{t}\left(x_{2}\right)\right|}{\left|1-\overline{\phi_{t}(w)} \phi_{t}\left(x_{3}\right)\right|\left|1-\overline{\phi_{t}(w)} \phi_{t}\left(x_{2}\right)\right|} .
$$

Moreover,

$$
\begin{aligned}
& \mathbb{P}^{\phi_{t}(w)}\left[\mathrm{BM} \text { hits } \partial \mathbb{U} \text { in }\left[\phi_{t}(\eta(t)), \phi_{t}\left(x_{2}\right)\right]\right] \\
= & \frac{1}{2 \pi}\left(\arg \frac{\phi_{t}\left(x_{2}\right)-\phi_{t}(w)}{1-\overline{\phi_{t}(w)} \phi_{t}\left(x_{2}\right)}-\arg \frac{\phi_{t}(\eta(t))-\phi_{t}(w)}{1-\overline{\phi_{t}(w)} \phi_{t}(\eta(t))}\right) \\
\geq & \frac{1}{2 \pi}\left|\frac{\phi_{t}\left(x_{2}\right)-\phi_{t}(w)}{1-\overline{\phi_{t}(w)} \phi_{t}\left(x_{2}\right)}-\frac{\phi_{t}(\eta(t))-\phi_{t}(w)}{1-\overline{\phi_{t}(w)} \phi_{t}(\eta(t))}\right| \\
= & \frac{1}{2 \pi} \frac{\left(1-\left|\phi_{t}(w)\right|^{2}\right)\left|\phi_{t}(\eta(t))-\phi_{t}\left(x_{2}\right)\right|}{\left|1-\overline{\phi_{t}(w)} \phi_{t}(\eta(t))\right|\left|1-\overline{\phi_{t}(w)} \phi_{t}\left(x_{2}\right)\right|} .
\end{aligned}
$$

Combining these two together, there exists $C>0$ such that

$$
\frac{\mathbb{P}^{\phi_{t}(w)}\left[\mathrm{BM} \text { hits } \partial \mathbb{U} \text { in }\left[\phi_{t}(\eta(t)), \phi_{t}\left(x_{2}\right)\right]\right]}{\mathbb{P}^{\phi_{t}(w)}\left[\mathrm{BM} \text { hits } \partial \mathbb{U} \text { in }\left[\phi_{t}\left(x_{2}\right), \phi_{t}\left(x_{3}\right)\right]\right]} \geq C \frac{\left|\phi_{t}(\eta(t))-\phi_{t}\left(x_{2}\right)\right|}{\left|\phi_{t}\left(x_{3}\right)-\phi_{t}\left(x_{2}\right)\right|} \frac{\left|\phi_{t}\left(x_{3}\right)-\phi_{t}(w)\right|}{\left|\phi_{t}(\eta(t))-\phi_{t}(w)\right|} .
$$

We denote the connected component of $\mathbb{H} \backslash\left(\eta[0, t] \cup C_{\delta}\right)$ which contains $\infty$ by $A$. By the relation between diameter and harmonic measure, there exists $C_{1}>0$, such that

$$
\begin{aligned}
\frac{1}{C_{1}} \operatorname{diam}\left(\phi_{\mathrm{t}}(\mathrm{A})\right) \leq \mathbb{P}^{0}\left[\text { BM hits } \phi_{\mathrm{t}}\left(\mathrm{C}_{\delta}\right) \text { before } \partial \mathbb{U}\right] & =\mathbb{P}^{z}\left[\text { BM hits } C_{\delta} \text { before } \partial(\mathbb{H} \backslash \eta[0, t])\right] \\
& \leq \mathbb{P}^{z}\left[\text { BM hits } C_{\delta} \text { before } \partial \mathbb{H}\right] \\
& \leq C_{1} \delta .
\end{aligned}
$$

Thus, we have

$$
\left|\phi_{t}(\eta(t))-\phi_{t}(w)\right| \leq C_{1}^{2} \delta
$$

For $\left|\phi_{t}(\eta(t))-\phi_{t}\left(x_{2}\right)\right|$ and $\left|\phi_{t}\left(x_{3}\right)-\phi_{t}(w)\right|$, we have

$$
\begin{aligned}
\left|\phi_{t}(\eta(t))-\phi_{t}\left(x_{2}\right)\right| & \geq \min \left\{\left|\phi_{t}\left(\frac{x_{4}+x_{3}}{2}\right)-\phi_{t}\left(x_{2}\right)\right|,\left|\phi_{t}\left(\frac{x_{1}+x_{0}}{2}\right)-\phi_{t}\left(x_{2}\right)\right|\right\}, \\
\left|\phi_{t}(w)-\phi_{t}\left(x_{3}\right)\right| & \geq\left|\phi_{t}(\eta(t))-\phi_{t}\left(x_{3}\right)\right|-\left|\phi_{t}(\eta(t))-\phi_{t}(w)\right| \\
& \geq \min \left\{\left|\phi_{t}\left(\frac{x_{4}+x_{3}}{2}\right)-\phi_{t}\left(x_{3}\right)\right|,\left|\phi_{t}\left(\frac{x_{1}+x_{0}}{2}\right)-\phi_{t}\left(x_{3}\right)\right|\right\}-C_{1}^{2} \delta .
\end{aligned}
$$




\section{Crossing probabilities in metric graph GFF}

Thus, by the uniform convergence, there exists $C_{2}>0$ such that

$$
\frac{\left|\phi_{t}(\eta(t))-\phi_{t}\left(x_{2}\right)\right|\left|\phi_{t}\left(x_{3}\right)-\phi_{t}(w)\right|}{\left|\phi_{t}\left(x_{3}\right)-\phi_{t}\left(x_{2}\right)\right|} \geq C_{2} .
$$

This implies that there exists $C>0$ such that

$$
\frac{\mathbb{P}^{\phi_{t}(w)}\left[\mathrm{BM} \text { hits } \partial \mathbb{U} \text { in }\left[\phi_{t}(\eta(t)), \phi_{t}\left(x_{2}\right)\right]\right]}{\mathbb{P}^{\phi_{t}(w)}\left[\mathrm{BM} \text { hits } \partial \mathbb{U} \text { in }\left[\phi_{t}\left(x_{2}\right), \phi_{t}\left(x_{3}\right)\right]\right]} \geq C \frac{1}{\delta} .
$$

Therefore, we have (A.2). Combining (A.1) and (A.2), we obtain the conclusion.

We set $\mathcal{B}_{\emptyset}=1$, and for $\alpha, \beta \in \mathrm{PP}_{\mathrm{N}}$ and $x_{1}<\cdots<x_{2 N}$, we define

$$
\mathcal{B}_{\beta}\left(x_{1}, \ldots, x_{2 N}\right):=\prod_{\{a, b\} \in \beta}\left|x_{a}-x_{b}\right|^{-1}, \quad F_{\alpha, \beta}\left(x_{1}, \ldots, x_{2 N}\right):=\frac{\mathcal{B}_{\beta}\left(x_{1}, \ldots, x_{2 N}\right)}{\mathcal{U}_{\alpha}\left(x_{1}, \ldots, x_{2 N}\right)^{2}} .
$$

Lemma A.3. Fix $\alpha, \beta \in \mathrm{PP}_{\mathrm{N}}$ such that $\alpha \stackrel{()}{\longleftarrow} \beta$. Fix $j \in\{1,2, \ldots, 2 N-1\}$, we assume that $\wedge^{j} \in \alpha$ and $\wedge^{j} \in \beta$. Fix $n \in\{1, \ldots, j-1, j+2, \ldots, 2 N\}$ such that $\alpha(n-1)=\alpha(j)$ and $\alpha(n)=\alpha(j-1)$. Fix $x_{1}<\cdots<x_{2 N}$. Suppose $\eta$ is a continuous simple curve in $\mathbb{H}$ starting from $x_{j}$ and terminating at $x_{n}$ at time $T$. Assume $\eta$ hits $\mathbb{R}$ only at its two end points. Let $\left(W_{t}, 0 \leq t \leq T\right)$ be its driving function and $\left(g_{t}, 0 \leq t \leq T\right)$ be the corresponding family of conformal maps. Then

$$
\lim _{t \rightarrow T} F_{\alpha, \beta}\left(g_{t}\left(x_{1}\right), \ldots, g_{t}\left(x_{j-1}\right), W_{t}, g_{t}\left(x_{j+1}\right), g_{t}\left(x_{2 N}\right)\right)=0 .
$$

Proof. We may assume $j+1<n$. The other case can be proved similarly. By definition, we have

$$
F_{\alpha, \beta}\left(x_{1}, \ldots, x_{2 N}\right)=\prod_{\substack{1 \leq i \leq 2 N \\ i \neq j, j+1}}\left|\frac{x_{i}-x_{j+1}}{x_{i}-x_{j}}\right|^{\vartheta_{\alpha}(i, j)} F_{\alpha / \wedge_{j}, \beta / \wedge_{j}}\left(x_{1}, \ldots, x_{j-1}, x_{j+2}, \ldots, x_{2 N}\right) .
$$

To get the conclusion, we will prove the following two estimates:

$$
\lim _{t \rightarrow T} \prod_{\substack{1 \leq i \leq 2 N \\ i \neq j, j+1}}\left|\frac{g_{t}\left(x_{i}\right)-g_{t}\left(x_{j+1}\right)}{g_{t}\left(x_{i}\right)-W_{t}}\right|^{\vartheta_{\alpha}(i, j)}=0,
$$

and

$$
\sup _{0 \leq t \leq T} F_{\alpha / \wedge_{j}, \beta / \wedge_{j}}\left(g_{t}\left(x_{1}\right), \ldots, g_{t}\left(x_{j-1}\right), g_{t}\left(x_{j+2}\right), \ldots, g_{t}\left(x_{2 N}\right)\right)<\infty .
$$

Suppose $\alpha=\left\{\left\{a_{1}, b_{1}\right\}, \ldots,\left\{a_{N}, b_{N}\right\}\right\}$ is ordered as in (2.2). The number of elements in two sets of indexes $A=\left\{i: j+1<i \leq n, i \in\left\{a_{1}, \ldots, a_{N}\right\}\right\}$ and $B=\{i: j+1<$ $\left.i \leq n, i \in\left\{b_{1}, \ldots, b_{N}\right\}\right\}$ are equal. Note that $n \in B$. We choose the increasing bijection $\xi: A \rightarrow B$ and suppose $\xi\left(i_{0}\right)=n$.

We first show (A.3). We write

$$
\begin{aligned}
& \prod_{\substack{1 \leq i \leq 2 N \\
i \neq j, j+1}}\left|\frac{g_{t}\left(x_{i}\right)-g_{t}\left(x_{j+1}\right)}{g_{t}\left(x_{i}\right)-W_{t}}\right|^{\vartheta_{\alpha}(i, j)} \\
= & \prod_{i<j \text { or } i>n}\left|\frac{g_{t}\left(x_{i}\right)-g_{t}\left(x_{j+1}\right)}{g_{t}\left(x_{i}\right)-W_{t}}\right|^{\vartheta_{\alpha}(i, j)} \times \prod_{\substack{i \in A \\
i \neq i_{0}}}\left(\left|\frac{g_{t}\left(x_{i}\right)-g_{t}\left(x_{j+1}\right)}{g_{t}\left(x_{i}\right)-W_{t}}\right|\left|\frac{g_{t}\left(x_{\xi(i)}\right)-W_{t}}{g_{t}\left(x_{\xi(i)}\right)-g_{t}\left(x_{j+1}\right)}\right|\right) \\
& \times\left|\frac{g_{t}\left(x_{i_{0}}\right)-g_{t}\left(x_{j+1}\right)}{g_{t}\left(x_{i_{0}}\right)-W_{t}}\right|\left|\frac{g_{t}\left(x_{n}\right)-W_{t}}{g_{t}\left(x_{n}\right)-g_{t}\left(x_{j+1}\right)}\right|
\end{aligned}
$$




\section{Crossing probabilities in metric graph GFF}

By Lemma A.1, we have

$$
\lim _{t \rightarrow T}\left|\frac{g_{t}\left(x_{i_{0}}\right)-g_{t}\left(x_{j+1}\right)}{g_{t}\left(x_{i_{0}}\right)-W_{t}}\right|\left|\frac{g_{t}\left(x_{n}\right)-W_{t}}{g_{t}\left(x_{n}\right)-g_{t}\left(x_{j+1}\right)}\right|=0 .
$$

By Lemma A.2, there exist $C_{1}, C_{2}>0$, which only depend on $\eta[0, T]$, such that for any $i \in A$ with $\xi(i) \neq n$, we have for all $t \in[0, T]$,

$$
C_{1} \leq\left|\frac{g_{t}\left(x_{i}\right)-g_{t}\left(x_{j+1}\right)}{g_{t}\left(x_{i}\right)-W_{t}}\right|\left|\frac{g_{t}\left(x_{\xi(i)}\right)-W_{t}}{g_{t}\left(x_{\xi(i)}\right)-g_{t}\left(x_{j+1}\right)}\right| \leq C_{2} .
$$

For $i \notin A \cup B$,

$$
\lim _{t \rightarrow T} \frac{g_{t}\left(x_{i}\right)-g_{t}\left(x_{j+1}\right)}{g_{t}\left(x_{i}\right)-W_{t}}=\frac{g_{T}\left(x_{i}\right)-g_{T}\left(x_{j+1}\right)}{g_{T}\left(x_{i}\right)-W_{T}} .
$$

Combining with (A.6) and (A.5), we obtain (A.3).

Next, we prove (A.4). We write

$$
\begin{aligned}
& F_{\alpha / \wedge_{j}, \beta / \wedge_{j}}\left(g_{t}\left(x_{1}\right), \ldots, g_{t}\left(x_{j-1}\right), g_{t}\left(x_{j+2}\right), \ldots, g_{t}\left(x_{2 N}\right)\right) \\
& \prod_{\left\{a_{i}, b_{i}\right\} \in \beta / \wedge_{j}}\left(g_{t}\left(x_{b_{i}}\right)-g_{t}\left(x_{a_{i}}\right)\right)^{-1} \\
& =\frac{a_{i} \notin A \text { or } b_{i} \notin B}{\prod_{\substack{i \notin A \cup B \\
\text { or } k \notin A \cup B}}\left|g_{t}\left(x_{k}\right)-g_{t}\left(x_{i}\right)\right|^{\vartheta_{\alpha}(i, k)}} \times S_{t},
\end{aligned}
$$

where

$$
S_{t}=\frac{\prod_{\substack{\left\{a_{i}, b_{i}\right\} \in \beta / \wedge_{j} \\ a_{i} \in A \text { and } b_{i} \in B}}\left(g_{t}\left(x_{b_{i}}\right)-g_{t}\left(x_{a_{i}}\right)\right)^{-1}}{\prod_{\substack{i \in A \cup B \backslash\{n\} \\ \text { and } k \in A \cup B \backslash\{n\}}}\left|g_{t}\left(x_{k}\right)-g_{t}\left(x_{i}\right)\right|^{\vartheta_{\alpha}(i, k)} \prod_{i \in A \cup B \backslash\{n\}}\left|g_{t}\left(x_{n}\right)-g_{t}\left(x_{i}\right)\right|^{\vartheta_{\alpha}(i, n)}} .
$$

In this decomposition, we have

$$
\frac{\prod_{\substack{\left\{a_{i}, b_{i}\right\} \in \beta / \wedge_{j} \\ a_{i} \notin A \text { or } \\ i}}\left(g_{t}\left(x_{b_{i}}\right)-g_{t}\left(x_{a_{i}}\right)\right)^{-1}}{\prod_{\substack{i \notin A \cup B \\ \text { or } k \notin A \cup B}}\left|g_{t}\left(x_{k}\right)-g_{t}\left(x_{i}\right)\right|^{\vartheta_{\alpha}(i, k)}} \asymp 1,
$$

because both the numerator and the denominator converge to a bounded and nonzero quantity as $t \rightarrow T$. Here the notation $\asymp$ is defined in the same way as in the proof of Lemma A.2. By (A.1), for distinct $i, k \in A \cup B \backslash\{n\}$, we have

$$
\left|g_{t}\left(x_{k}\right)-g_{t}\left(x_{i}\right)\right| \asymp g_{t}\left(x_{j+2}\right)-g_{t}\left(x_{j+1}\right) .
$$

By the same method as in the proof of (A.2), for $i \in A \cup B \backslash\{n\}$, we have

$$
\lim _{t \rightarrow T} \frac{g_{t}\left(x_{n}\right)-g_{t}\left(x_{i}\right)}{g_{t}\left(x_{n}\right)-g_{t}\left(x_{n-1}\right)}=1 .
$$

Thus we have

$$
S_{t} \asymp \prod_{\substack{\left\{a_{i}, b_{i}\right\} \in \beta / \wedge_{j} \\ a_{i} \in A \text { and } b_{i} \in B}}\left(g_{t}\left(x_{b_{i}}\right)-g_{t}\left(x_{a_{i}}\right)\right)^{-1}\left(g_{t}\left(x_{j+2}\right)-g_{t}\left(x_{j+1}\right)\right)^{\# A-1}\left(g_{t}\left(x_{n}\right)-g_{t}\left(x_{n-1}\right)\right) .
$$

When there is $a \in A$ such that $\{a, n\} \in \beta / \wedge_{j}$, and $\# A-1$ pairs $\left\{a_{i}, b_{i}\right\} \in \beta / \wedge_{j}$ such that $a_{i} \in A$ and $b_{i} \in B$, we have $S_{t} \asymp 1$. Otherwise, we have $\lim _{t \rightarrow T} S_{t}=0$. This gives (A.4) and completes the proof. 


\section{B Proof of Proposition 5.6}

Proof of Proposition 5.6. From (3.12), we have

$$
\mathcal{Z}_{\alpha}\left(x_{1}, \ldots, x_{4 N}\right)=\sum_{\beta \in \mathrm{DP}_{2 \mathrm{~N}}} \mathcal{M}_{\alpha, \beta}^{-1} \mathcal{U}_{\beta}\left(x_{1}, \ldots, x_{4 N}\right) .
$$

For $\beta \in \mathrm{DP}_{2 \mathrm{~N}}$, there exists $J \subset\{1,2, \ldots, 2 N\}$ such that

$$
\diamond_{2 j-1} \in \beta \text { for all } j \in J \text {, and } \times_{2 j-1} \in \beta \text { for all } j \in\{1, \ldots, 2 N\} \backslash J \text {. }
$$

Then, from the definition (3.10), the following limit exists:

$$
\lim _{\substack{x_{2 j-1}, x_{2 j} \rightarrow y_{j} \\ \forall j \notin J}}, \frac{\mathcal{U}_{\beta}\left(x_{1}, \ldots, x_{4 N}\right)}{\prod_{j \notin J}\left(x_{2 j}-x_{2 j-1}\right)^{1 / 2}} .
$$

To obtain the desired limit, we need to group distinct $\beta$ 's according to the location of their local extremes.

Let $J$ be any subset of $\{1,2, \ldots, 2 N\}$, and define

$$
\mathcal{P}_{J}^{\alpha}=\left\{\beta \in \mathrm{DP}_{2 \mathrm{~N}}: \beta \succeq \alpha, \diamond_{2 \mathrm{j}-1} \in \beta \text { for all } \mathrm{j} \in \mathrm{J}, \times_{2 \mathrm{j}-1} \in \beta \text { for all } \mathrm{j} \in\{1,2, \ldots, 2 \mathrm{~N}\} \backslash \mathrm{J}\right\} .
$$

It suffices to show that the following limit exists for all possible $J$ :

$$
\lim _{\substack{x_{2 j-1}, x_{2 j} \rightarrow y_{j} \\ \forall j \in J}} \frac{\sum_{\beta \in \mathcal{P}_{J}^{\alpha}} \mathcal{M}_{\alpha, \beta}^{-1} \mathcal{U}_{\beta}\left(x_{1}, \ldots, x_{4 N}\right)}{\prod_{j \in J}\left(x_{2 j}-x_{2 j-1}\right)^{1 / 2}} .
$$

Suppose $n=\# J \geq 1$. For some $\beta_{0} \in \mathcal{P}_{J}^{\alpha}$ such that $\vee_{2 j-1} \in \beta_{0}$ for all $j \in J$, we define

$$
\mathcal{P}_{J}^{\alpha, \beta_{0}}=\left\{\beta \in \mathrm{DP}_{2 \mathrm{~N}}: \exists\left\{\mathrm{i}_{1}, \ldots, \mathrm{i}_{\mathrm{k}}\right\} \subset \mathrm{J} \text { such that } \beta=\beta_{0} \uparrow \diamond_{2 \mathrm{i}_{1}-1} \cdots \uparrow \diamond_{2 \mathrm{i}_{\mathrm{k}}-1}\right\} .
$$

It is clear that $\# \mathcal{P}_{J}^{\alpha, \beta_{0}}=2^{n}$. Furthermore, for distinct $\beta_{0}, \beta_{0}^{\prime} \in \mathcal{P}_{J}^{\alpha}$ such that $\vee_{2 j-1} \in \beta_{0}$ and $\vee_{2 j-1} \in \beta_{0}^{\prime}$ for all $j \in J$, we see that $\mathcal{P}_{J}^{\alpha, \beta_{0}} \cap \mathcal{P}_{J}^{\alpha, \beta_{0}^{\prime}}=\emptyset$. Thus $\left\{\mathcal{P}_{J}^{\alpha, \beta_{0}}: \beta_{0} \in\right.$ $\mathcal{P}_{J}^{\alpha}$ with $\left.\vee_{2 j-1} \in \beta_{0} \forall j \in J\right\}$ gives a disjoint partition of $\mathcal{P}_{J}^{\alpha}$. Therefore, it suffices to show that the following limit exists for each such $\beta_{0}$ :

$$
\lim _{\substack{x_{2 j-1}, x_{2 j} \rightarrow y_{j} \\ \forall j \in J}} \frac{\sum_{\beta \in \mathcal{P}_{J}^{\alpha, \beta_{0}}} \mathcal{M}_{\alpha, \beta}^{-1} \mathcal{U}_{\beta}\left(x_{1}, \ldots, x_{4 N}\right)}{\prod_{j \in J}\left(x_{2 j}-x_{2 j-1}\right)^{1 / 2}} .
$$

To derive (B.1), we will show a more general conclusion. Suppose $K \subset J$ and suppose $\gamma_{0} \in \mathcal{P}_{J}^{\alpha}$ such that $\vee_{2 j-1} \in \gamma_{0}$ for all $j \in K$. We define

$$
\mathcal{P}_{J, K}^{\alpha, \gamma_{0}}=\left\{\gamma \in \mathrm{DP}_{2 \mathrm{~N}}: \exists\left\{\mathrm{i}_{1}, \ldots, \mathrm{i}_{\mathrm{k}}\right\} \subset \mathrm{K} \text { such that } \gamma=\gamma_{0} \uparrow \diamond_{2 \mathrm{i}_{1}-1} \cdots \uparrow \diamond_{2 \mathrm{i}_{\mathrm{k}}-1}\right\} .
$$

We denote $R_{K}:=\{2 j-1,2 j: j \in K\}$, and we denote

$$
Z_{K, j}^{\gamma}:=\sum_{l \notin R_{K}} \frac{\vartheta_{\gamma}(l, 2 j-1)}{x_{l}-y_{j}} .
$$

We denote by $\mathfrak{S}_{n}$ the set of permutations of $\{1,2, \ldots, n\}$. Suppose $K=\left\{j_{1}, \ldots, j_{n}\right\}$. For any $\gamma_{0} \in \mathcal{P}_{J}^{\alpha}$ such that $\vee_{2 j-1} \in \gamma_{0}$ for all $j \in K$, we claim that

$$
\begin{aligned}
& \lim _{\substack{x_{2 j-1}, x_{2 j} \rightarrow y_{j} \\
\forall j \in K}} \frac{\sum_{\gamma \in \mathcal{P}_{J, K}^{\alpha, \gamma_{0}}} \mathcal{M}_{\alpha, \gamma}^{-1} \mathcal{H}_{\gamma}\left(x_{1}, \ldots, x_{4 N}\right)}{\prod_{j \in K}\left(x_{2 j}-x_{2 j-1}\right)^{1 / 2}} \\
= & \mathcal{M}_{\alpha, \gamma_{0}}^{-1} \prod_{\substack{1 \leq t<s \leq 4 N \\
t, s \notin R_{K}}}\left(x_{s}-x_{t}\right)^{\frac{1}{2} \vartheta_{\gamma_{0}}(t, s)} \times\left(\sum_{m=0}^{\left\lfloor\frac{n}{2}\right\rfloor} \sum_{\sigma \in \mathfrak{J}_{n}^{m}} \frac{2^{m} Z_{K, j_{\sigma_{2 m+1}}}^{\gamma_{0}} \cdots Z_{K, j_{\sigma_{n}}}^{\gamma_{0}}}{\left(y_{j_{\sigma_{1}}}-y_{j_{\sigma_{2}}}\right)^{2} \times \cdots \times\left(y_{j_{\sigma_{2 m-1}}}-y_{j_{\sigma_{2 m}}}\right)^{2}}\right),
\end{aligned}
$$




\section{Crossing probabilities in metric graph GFF}

where $\mathfrak{J}_{n}^{m}$ is a subset of $\mathfrak{S}_{n}$ :

$\mathfrak{J}_{n}^{m}=\left\{\sigma \in \mathfrak{S}_{n}: \sigma_{1}<\sigma_{3}<\cdots<\sigma_{2 m-1}\right.$, and $\sigma_{2 j-1}<\sigma_{2 j}$ for $j \leq m$, and $\left.\sigma_{2 m+1}<\cdots<\sigma_{n}\right\}$.

Fix $\alpha$ and $J$, we will show (B.2) by induction on $n=\# K$. It is true for $K=\emptyset$ as it is the same as the definition of $\mathcal{U}_{\gamma_{0}}$. Suppose (B.2) holds for $\# K=i$. We need to show it for $\# K=i+1$. Suppose $K=\left\{j_{1}, \ldots, j_{i+1}\right\}$. We will take the limit in the left hand side of (B.2) in a particular order: we first let $x_{2 j-1}, x_{2 j} \rightarrow y_{j}$ with $j \in K \backslash\left\{j_{i+1}\right\}$ and then let $x_{2 j_{i+1}-1}, x_{2 j_{i+1}} \rightarrow y_{j_{i+1}}$. It will be clear from the calculation that the limit in (B.2) for $\# K=i+1$ does not depend on the order of taking limits.

For any $\gamma_{0} \in \mathcal{P}_{J}^{\alpha}$ such that $\vee_{2 j_{1}-1}, \ldots, \vee_{2 j_{i+1}-1} \in \gamma_{0}$, we have the decomposition

$$
\mathcal{P}_{J, K}^{\alpha, \gamma_{0}}=\mathcal{P}_{J, K \backslash\left\{j_{i+1}\right\}}^{\alpha, \gamma_{0}} \bigsqcup \mathcal{P}_{J, K \backslash\left\{j_{i+1}\right\}}^{\alpha, \gamma_{0} \uparrow \diamond_{2 j_{i+1}-1}} .
$$

Denote by $\gamma_{1}=\gamma_{0} \uparrow \diamond_{2 j_{i+1}-1}$ and $K_{1}=\left\{j_{1}, \ldots, j_{i}\right\}=K \backslash\left\{j_{i+1}\right\}$. Then we have

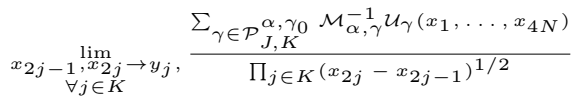

$$
\begin{aligned}
& =\lim _{x_{2 j_{i+1}-1}, x_{2 j_{i+1}} \rightarrow y_{j+1}} \frac{1}{\left(x_{2 j_{i+1}}-x_{2 j_{i+1}-1}\right)^{1 / 2}}
\end{aligned}
$$

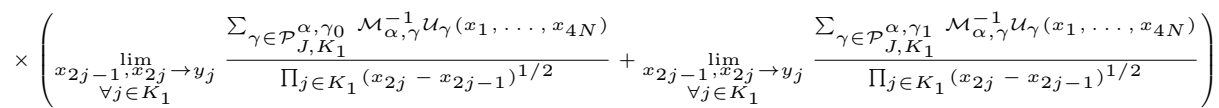

By the induction hypothesis, we have

$$
\begin{aligned}
& \lim _{\substack{x_{2 j}, x_{2 j} \rightarrow y_{j} \\
\forall j \in K_{1}}} \frac{\sum_{\gamma \in \mathcal{P}_{J, K_{1}}^{\alpha, \gamma_{0}}} \mathcal{M}_{\alpha, \gamma}^{-1} \mathcal{U}_{\gamma}\left(x_{1}, \ldots, x_{4 N}\right)}{\prod_{j \in K_{1}}\left(x_{2 j}-x_{2 j-1}\right)^{1 / 2}}=\mathcal{M}_{\alpha, \gamma_{0}}^{-1} \prod_{\substack{1 \leq t<s \leq 4 N \\
t, s \notin R_{K_{1}}}}\left(x_{s}-x_{t}\right)^{\frac{1}{2} \vartheta_{\gamma_{0}}(t, s)} \times S_{0}, \\
& \lim _{\substack{x_{2 j}-x_{2 j} \rightarrow y_{j} \\
\forall j \in K_{1}}} \frac{\sum_{\gamma \in \mathcal{P}_{J, K_{1}}^{\alpha, \gamma_{1}}} \mathcal{M}_{\alpha, \gamma}^{-1} \mathcal{U}_{\gamma}\left(x_{1}, \ldots, x_{4 N}\right)}{\prod_{j \in K_{1}}\left(x_{2 j}-x_{2 j-1}\right)^{1 / 2}}=\mathcal{M}_{\alpha, \gamma_{1}}^{-1} \prod_{\substack{1 \leq t<s \leq 4 N \\
t, s \notin R_{K_{1}}}}\left(x_{s}-x_{t}\right)^{\frac{1}{2} \vartheta_{\gamma_{1}}(t, s)} \times S_{1},
\end{aligned}
$$

where

$$
S_{u}=\sum_{m=0}^{\left\lfloor\frac{i}{2}\right\rfloor} \sum_{\sigma \in \mathfrak{J}_{i}^{m}} \frac{2^{m} Z_{K_{1}, j_{\sigma_{2 m+1}}}^{\gamma_{u}} \cdots Z_{K_{1}, j_{\sigma_{i}}}^{\gamma_{u}}}{\left(y_{\sigma_{\sigma_{1}}}-y_{j_{\sigma_{2}}}\right)^{2} \times \cdots \times\left(y_{j_{\sigma_{2 m-1}}}-y_{j_{\sigma_{2 m}}}\right)^{2}}, \quad \text { for } u=0,1
$$

Comparing the two expressions in the right hand side, we have $\mathcal{M}_{\alpha, \gamma_{1}}^{-1}=-\mathcal{M}_{\alpha, \gamma_{0}}^{-1}$, and

$$
\begin{aligned}
& \prod_{\substack{1 \leq t<s \leq 4 N \\
t, s \notin R_{K_{1}}}}\left(x_{s}-x_{t}\right)^{\frac{1}{2} \vartheta_{\gamma_{0}}(t, s)} \\
= & \prod_{\substack{1 \leq t<s \leq 4 N \\
t, s \notin R_{K}}}\left(x_{s}-x_{t}\right)^{\frac{1}{2} \vartheta_{\gamma_{0}}(t, s)} \times \prod_{\substack{1 \leq n \leq 4 N \\
n \notin R_{K}}}\left|\frac{x_{n}-x_{2 j_{i+1}-1}}{x_{n}-x_{2 j_{i+1}}}\right|^{\frac{1}{2} \vartheta_{\gamma_{0}}\left(n, 2 j_{i+1}-1\right)} \times\left(x_{2 j_{i+1}}-x_{2 j_{i+1}-1}\right)^{-\frac{1}{2}} \\
& \prod_{\substack{1 \leq t<s \leq 4 N \\
t, s \notin R_{K_{1}}}}\left(x_{s}-x_{t}\right)^{\frac{1}{2} \vartheta_{\gamma_{1}}(t, s)} \\
= & \prod_{\substack{1 \leq t<s \leq 4 N \\
t, s \notin R_{K}}}\left(x_{s}-x_{t}\right)^{\frac{1}{2} \vartheta_{\gamma_{0}}(t, s)} \times \prod_{\substack{1 \leq n \leq 4 N \\
n \notin R_{K}}}\left|\frac{x_{n}-x_{2 j_{i+1}-1}}{x_{n}-x_{2 j_{i+1}}}\right|^{-\frac{1}{2} \vartheta_{\gamma_{0}}\left(n, 2 j_{i+1}-1\right)} \times\left(x_{2 j_{i+1}}-x_{2 j_{i+1}-1}\right)^{-\frac{1}{2}}
\end{aligned}
$$




\section{Crossing probabilities in metric graph GFF}

Plugging into (B.3) and denoting $\delta=x_{2 j_{i+1}}-x_{2 j_{i+1}-1}$, we have

$$
\begin{aligned}
& \lim _{\substack{x_{2 j-1}, x_{2 j} \rightarrow y_{j}, \forall j \in K}} \frac{\sum_{\gamma \in \mathcal{P}_{J, K}^{\alpha, \gamma_{0}}} \mathcal{M}_{\alpha, \gamma}^{-1} \mathcal{U}_{\gamma}\left(x_{1}, \ldots, x_{4 N}\right)}{\prod_{j \in K}\left(x_{2 j}-x_{2 j-1}\right)^{1 / 2}} \\
= & \mathcal{M}_{\alpha, \gamma_{0}}^{-1} \prod_{\substack{1 \leq t<s \leq 4 N \\
t, s \notin R_{K}}}\left(x_{s}-x_{t}\right)^{\frac{1}{2} \vartheta_{\gamma_{0}}(t, s)} \\
& \times \lim _{\substack{\delta \rightarrow 0 \\
x_{2 j_{i+1}-1} \rightarrow y_{j_{i+1}}}} \frac{1}{\delta}\left(\left(\prod_{\substack{1 \leq n \leq 4 N \\
n \notin \bar{R}_{K}}}\left|\frac{x_{n}-x_{2 j_{i+1}-1}}{x_{n}-x_{2 j_{i+1}}}\right|^{\vartheta_{\gamma_{0}}\left(n, 2 j_{i+1}-1\right)}-1\right) \times S_{0}+S_{0}-S_{1}\right) .
\end{aligned}
$$

Note that

$$
\begin{aligned}
& \left.Z_{K_{1}, j_{\sigma_{2 m+1}}}^{\gamma_{0}} \cdots Z_{K_{1}, j_{\sigma_{i}}}^{\gamma_{0}}+\frac{\delta}{\left(x_{2 j_{i+1}-1}-y_{j_{\sigma_{2 m+1}}}\right)\left(x_{2 j_{i+1}}-y_{j_{\sigma_{2 m+1}}}\right)}\right) \\
& \times \cdots \times\left(Z_{K, j_{\sigma_{i}}}^{\gamma_{0}}+\frac{\delta}{\left(x_{2 j_{i+1}-1}-y_{j_{i}}\right)\left(x_{2 j_{i+1}}-y_{j_{i}}\right)}\right) ; \\
& \left.Z_{K_{1}, j_{\sigma_{2 m+1}}}^{\gamma_{1}} \cdots Z_{K_{1}, j_{\sigma_{i}}}^{\gamma_{1}}\right) \\
= & \left(Z_{K, j_{\sigma_{2 m+1}}}^{\gamma_{0}}-\frac{\delta}{\left(x_{2 j_{i+1}-1}-y_{j_{\sigma_{2 m+1}}}\right)\left(x_{2 j_{i+1}}-y_{j_{2 m+1}}\right)}\right) \\
& \times \cdots \times\left(Z_{K, j_{\sigma_{i}}}^{\gamma_{0}}-\frac{\delta}{\left(x_{2 j_{i+1}-1}-y_{j_{i}}\right)\left(x_{2 j_{i+1}}-y_{j_{i}}\right)}\right)
\end{aligned}
$$

Plugging into $S_{0}$ and $S_{1}$, we have

$$
\begin{aligned}
& \lim _{\substack{\delta \rightarrow 0 \\
x_{2 j_{i+1}-1} \rightarrow y_{j_{i+1}}}} \frac{1}{\delta}\left(\prod_{\substack{1 \leq n \leq 4 N \\
n \notin \mathbb{R} K}}\left|\frac{x_{n}-x_{2 j_{i+1}-1}}{x_{n}-x_{2 j_{i+1}}}\right|^{\vartheta_{\gamma_{0}}\left(n, 2 j_{i+1}-1\right)}-1\right) \times S_{0} \\
& =Z_{K, j_{i+1}}^{\gamma_{0}}\left(\sum_{m=0}^{\left\lfloor\frac{i}{2}\right\rfloor} \sum_{\sigma \in \mathfrak{J}_{i}^{m}} \frac{2^{m} Z_{K, j_{\sigma_{2 m+1}}}^{\gamma_{0}} \cdots Z_{K, j_{\sigma_{i}}}^{\gamma_{0}}}{\left(y_{j_{\sigma_{1}}}-y_{j_{\sigma_{2}}}\right)^{2} \times \cdots \times\left(y_{j_{\sigma_{2 m-1}}}-y_{j_{\sigma_{2 m}}}\right)^{2}}\right) ; \\
& \lim _{\substack{\delta \rightarrow 0 \\
x_{2 j_{i+1}-1} \rightarrow y_{j_{i+1}}}} \frac{1}{\delta}\left(S_{0}-S_{1}\right) \\
& =\sum_{m=0}^{\left\lfloor\frac{i}{2}\right\rfloor} \sum_{\sigma \in \mathfrak{J}_{i}^{m}} \sum_{r=2 m+1}^{i} \frac{2^{m+1} Z_{K, j_{\sigma_{2 m+1}}}^{\gamma_{0}} \cdots Z_{K, j_{\sigma_{r-1}}}^{\gamma_{0}} Z_{K, j_{\sigma_{r+1}}}^{\gamma_{0}} \cdots Z_{K, j_{\sigma_{i}}}^{\gamma_{0}}}{\left(y_{j_{\sigma_{1}}}-y_{j_{\sigma_{2}}}\right)^{2} \times \cdots \times\left(y_{j_{\sigma_{2 m-1}}}-y_{j_{\sigma_{2 m}}}\right)^{2} \times\left(y_{j_{i+1}}-y_{j_{\sigma_{r}}}\right)^{2}} .
\end{aligned}
$$




\section{Crossing probabilities in metric graph GFF}

Plugging into (B.4), we see that it remains to show

$$
\begin{aligned}
& \sum_{m=0}^{\left\lfloor\frac{i+1}{2}\right\rfloor} \sum_{\tau \in \mathfrak{J}_{i+1}^{m}} \frac{2^{m} Z_{K, j_{\tau_{2 m+1}}}^{\gamma_{0}} \cdots Z_{K, j_{\tau_{i+1}}}^{\gamma_{0}}}{\left(y_{j_{\tau_{1}}}-y_{j_{\tau_{2}}}\right)^{2} \times \cdots \times\left(y_{j_{\tau_{2 m}-1}}-y_{j_{\tau_{2 m}}}\right)^{2}} \\
= & \sum_{m=0}^{\left\lfloor\frac{i}{2}\right\rfloor} \sum_{\sigma \in \mathfrak{J}_{i}^{m}}\left(\sum_{r=2 m+1}^{i} \frac{2^{m+1} Z_{K, j_{\sigma_{2 m+1}}}^{\gamma_{0}} \cdots Z_{K, j_{\sigma_{r-1}}}^{\gamma_{0}} Z_{K, j_{\sigma_{r+1}}}^{\gamma_{0}} \cdots Z_{K, j_{\sigma_{i}}}^{\gamma_{0}}}{\left(y_{j_{\sigma_{1}}}-y_{j_{\sigma_{2}}}\right)^{2} \times \cdots \times\left(y_{j_{\sigma_{2 m-1}}}-y_{j_{\sigma_{2 m}}}\right)^{2} \times\left(y_{j_{i+1}}-y_{j_{\sigma_{r}}}\right)^{2}}\right) \\
& +\sum_{m=0}^{\left\lfloor\frac{i}{2}\right\rfloor} \sum_{\sigma \in \mathfrak{J}_{i}^{m}}\left(\frac{2^{m} Z_{K, j_{\sigma_{2 m+1}}}^{\gamma_{0}} \cdots Z_{K, j_{\sigma_{i}}}^{\gamma_{0}} Z_{K, j_{i+1}}^{\gamma_{0}}}{\left(y_{j_{\sigma_{1}}}-y_{j_{\sigma_{2}}}\right)^{2} \times \cdots \times\left(y_{j_{\sigma_{2 m-1}}}-y_{j_{\sigma_{2 m}}}\right)^{2}}\right) .
\end{aligned}
$$

For $\tau \in \mathfrak{J}_{i+1}^{m}$, let us consider the location of $i+1$ in $\tau$. If $\tau_{i+1}=i+1$, we define $\sigma_{j}=\tau_{j}$ for $1 \leq j \leq i$, then $\sigma \in \mathfrak{J}_{i}^{m}$. Thus, for $0 \leq m \leq\left\lfloor\frac{i}{2}\right\rfloor$, we have

$$
\begin{aligned}
& \sum_{\substack{\tau \in \mathfrak{J}_{i+1}^{m}: \\
\tau_{i+1}=i+1}} \frac{2^{m} Z_{K, j_{\tau_{2 m+1}}}^{\gamma_{0}} \cdots Z_{K, j_{\tau_{i+1}}}^{\gamma_{0}}}{\left(y_{j_{\tau_{1}}}-y_{j_{\tau_{2}}}\right)^{2} \times \cdots \times\left(y_{j_{\tau_{2 m-1}}}-y_{j_{\tau_{2 m}}}\right)^{2}} \\
= & \sum_{\sigma \in \mathfrak{J}_{i}^{m}} \frac{2^{m} Z_{K, j_{\sigma_{2 m+1}}}^{\gamma_{0}} \cdots Z_{K, j_{\sigma_{i}}}^{\gamma_{0}} Z_{K, j_{i+1}}^{\gamma_{0}}}{\left(y_{j_{\sigma_{1}}}-y_{j_{\sigma_{2}}}\right)^{2} \times \cdots \times\left(y_{j_{\sigma_{2 m-1}}}-y_{j_{\sigma_{2 m}}}\right)^{2}} .
\end{aligned}
$$

If $\tau_{i+1}<i+1$, we define a mapping for each $m \in\left\{1,2, \ldots,\left\lfloor\frac{i}{2}\right\rfloor\right\}$ :

$$
T_{m}:\left\{\tau \in \mathfrak{J}_{i+1}^{m}: \tau_{i+1}<i+1\right\} \longrightarrow \mathfrak{J}_{i}^{m-1} \times\{2 m-1, \ldots, i\}, \quad \tau \mapsto(\sigma, r)
$$

in the following way. For $\tau \in \mathfrak{J}_{i+1}^{m}$ and $\tau_{i+1}<i+1$, we must have $\tau_{2 k}=i+1$ for some $1 \leq k \leq m$. We set $\sigma_{j}=\tau_{j}$, for $j \leq 2 k-2$; we set $\sigma_{j}=\tau_{j+2}$, for $2 k-1 \leq j \leq 2 m-2$; and we set $\left\{\sigma_{2 m-1}, \ldots, \sigma_{i}\right\}=\left\{\tau_{2 k-1}, \tau_{2 m+1}, \ldots, \tau_{i+1}\right\}$ such that $\sigma_{2 m-1}<\ldots<\sigma_{i}$. Suppose $\sigma_{r}=\tau_{2 k-1}$ for some $r \in\{2 m-1, \ldots, i\}$. This defines the map $T_{m}(\tau)=(\sigma, r)$. We argue that $T_{m}$ is a bijection. For any $(\sigma, r) \in \mathfrak{J}_{i}^{m-1} \times\{2 m-1, \ldots, i\}$, we can define $\tau$ as follows: $\left\{\left\{\tau_{1}, \tau_{2}\right\}, \ldots,\left\{\tau_{2 m-1}, \tau_{2 m}\right\}\right\}=\left\{\left\{\sigma_{1}, \sigma_{2}\right\}, \ldots,\left\{\sigma_{2 m-3}, \sigma_{2 m-2}\right\},\left\{\sigma_{r}, i+1\right\}\right\}$ and $\left\{\tau_{2 m+1}, \ldots, \tau_{i+1}\right\}=\left\{\sigma_{2 m-1}, \ldots, \sigma_{r-1}, \sigma_{r+1}, \ldots, \sigma_{i}\right\}$, such that $\tau_{1}<\tau_{3}<\cdots<\tau_{2 m-1}$, $\tau_{2 j-1}<\tau_{2 j}$ for $j \leq m$ and $\tau_{2 m+1}<\cdots<\tau_{i+1}$. Then we have $\tau \in \mathfrak{J}_{i+1}^{m}$ and $\tau_{i+1}<i+1$. This implies $T_{m}$ is a bijection. Thus, we have

$$
\begin{aligned}
& \sum_{m=1}^{\left\lfloor\frac{i}{2}\right\rfloor} \sum_{\substack{\tau \in \mathfrak{J}_{i+1}^{m}: \\
\tau_{i+1}<i+1}} \frac{2^{m} Z_{K, j_{\tau_{2 m+1}}}^{\gamma_{0}} \cdots Z_{K, j_{\tau_{i+1}}}^{\gamma_{0}}}{\left(y_{j_{\tau_{1}}}-y_{j_{\tau_{2}}}\right)^{2} \times \cdots \times\left(y_{j_{\tau_{2 m-1}}}-y_{j_{\tau_{2 m}}}\right)^{2}} \\
= & \sum_{m=1}^{\left\lfloor\frac{i}{2}\right\rfloor} \sum_{\sigma \in \mathfrak{J}_{i}^{m-1}} \sum_{r=2 m-1}^{i} \frac{2^{m} Z_{K, j_{\sigma_{2 m-1}}}^{\gamma_{0}} \cdots Z_{K, j_{\sigma_{r-1}}}^{\gamma_{0}} Z_{K, j_{\sigma_{r+1}}}^{\gamma_{0}} \cdots Z_{K, j_{\sigma_{i}}}^{\gamma_{0}}}{\left(y_{j_{\sigma_{1}}}-y_{j_{\sigma_{2}}}\right)^{2} \times \cdots \times\left(y_{j_{\sigma_{2 m-3}}}-y_{j_{\sigma_{2 m-2}}}\right)^{2} \times\left(y_{j_{i+1}}-y_{j_{\sigma}}\right)^{2}} \\
= & \sum_{m=0}^{\left\lfloor\frac{i}{2}\right\rfloor-1} \sum_{\sigma \in \mathfrak{J}_{i}^{m}} \sum_{r=2 m+1}^{i} \frac{2^{m+1} Z_{K, j_{\sigma_{2 m+1}}}^{\gamma_{0}} \cdots Z_{K, j_{\sigma_{r-1}}}^{\gamma_{0}} Z_{K, j_{\sigma_{r+1}}}^{\gamma_{0}} \cdots Z_{K, j_{\sigma_{i}}}^{\gamma_{0}}}{\left(y_{j_{\sigma_{1}}}-y_{j_{\sigma_{2}}}\right)^{2} \times \cdots \times\left(y_{j_{\sigma_{2 m-1}}}-y_{j_{\sigma_{2 m}}}\right)^{2} \times\left(y_{j_{i+1}}-y_{j_{\sigma_{r}}}\right)^{2}} .
\end{aligned}
$$

Combining (B.6) and (B.7), we obtain (B.5) for even $i$. Next, suppose $i$ is odd and denote 
$\ell=\frac{i+1}{2}$. By (B.6) and (B.7), we have

$$
\begin{aligned}
& \sum_{m=0}^{\ell} \sum_{\substack{\tau \in \mathfrak{J}_{i+1}^{m}: \\
\tau_{i+1}=i+1}} \frac{2^{m} Z_{K, j_{\tau_{2 m+1}}}^{\gamma_{0}} \cdots Z_{K, j_{\tau_{i+1}}}^{\gamma_{0}}}{\left(y_{j_{\tau_{1}}}-y_{j_{\tau_{2}}}\right)^{2} \times \cdots \times\left(y_{j_{\tau_{2 m-1}}}-y_{j_{\tau_{2 m}}}\right)^{2}} \\
& =\sum_{m=0}^{\left\lfloor\frac{i}{2}\right\rfloor} \sum_{\sigma \in \mathfrak{J}_{i}^{m}} \frac{2^{m} Z_{K, j_{\sigma_{2 m+1}}}^{\gamma_{0}} \cdots Z_{K, j_{\sigma_{i}}}^{\gamma_{0}} Z_{K, j_{i+1}}^{\gamma_{0}}}{\left(y_{j_{\sigma_{1}}}-y_{j_{\sigma_{2}}}\right)^{2} \times \cdots \times\left(y_{j_{\sigma_{2 m-1}}}-y_{j_{\sigma_{2 m}}}\right)^{2}} \\
& +\sum_{\substack{\tau \in \mathfrak{J}_{i+1}^{\ell}: \\
\tau_{i+1}=i+1}} \frac{2^{\ell}}{\left(y_{j_{\tau_{1}}}-y_{j_{\tau_{2}}}\right)^{2} \times \cdots \times\left(y_{j_{i}}-y_{j_{\tau_{i+1}}}\right)^{2}} ; \\
& \sum_{m=0}^{\ell} \sum_{\substack{\tau \in \mathfrak{I}_{i+1}^{m}: \\
\tau_{i+1}<i+1}} \frac{2^{m} Z_{K, j_{\tau_{2 m+1}}}^{\gamma_{0}} \cdots Z_{K, j_{\tau_{i+1}}}^{\gamma_{0}}}{\left(y_{j_{\tau_{1}}}-y_{j_{\tau_{2}}}\right)^{2} \times \cdots \times\left(y_{j_{\tau_{2 m-1}}}-y_{j_{\tau_{2}}}\right)^{2}} \\
& =\sum_{m=1}^{\left\lfloor\frac{i}{2}\right\rfloor} \sum_{\substack{\tau \in \mathfrak{J}_{i+1}^{m}: \\
\tau_{i+1}<i+1}} \frac{2^{m} Z_{K, j_{\tau_{2 m+1}}}^{\gamma_{0}} \cdots Z_{K, j_{\tau_{i+1}}}^{\gamma_{0}}}{\left(y_{\tau_{\tau_{1}}}-y_{j_{\tau_{2}}}\right)^{2} \times \cdots \times\left(y_{j_{\tau_{2 m}-1}}-y_{j_{\tau_{2}}}\right)^{2}} \\
& +\sum_{\substack{\tau \in \mathfrak{J}_{i+1}^{\ell}: \\
\tau_{i+1}<i+1}} \frac{2^{\ell}}{\left(y_{j_{1}}-y_{j_{\tau_{2}}}\right)^{2} \times \cdots \times\left(y_{j_{\tau_{i}}}-y_{j_{\tau_{i+1}}}\right)^{2}} \\
& =\sum_{m=0}^{\left\lfloor\frac{i}{2}\right\rfloor-1} \sum_{\sigma \in \mathfrak{J}_{i}^{m}} \sum_{r=2 m+1}^{i} \frac{2^{m+1} Z_{K, j_{\sigma_{2 m+1}}}^{\gamma_{0}} \cdots Z_{K, j_{\sigma_{r-1}}}^{\gamma_{0}} Z_{K, j_{\sigma_{r+1}}}^{\gamma_{\sigma_{2}}} \cdots Z_{K, j_{\sigma_{i}}}^{\gamma_{0}}}{\left(y_{j_{\sigma_{1}}}-y_{j_{\sigma_{2}}}\right)^{2} \times \cdots \times\left(y_{j_{\sigma_{2 m-1}}}-y_{j_{\sigma_{2 m}}}\right)^{2} \times\left(y_{j_{i+1}}-y_{j_{\sigma_{r}}}\right)^{2}} \\
& +\sum_{\substack{\tau \in \mathcal{J}_{i+1}^{\ell}: \\
\tau_{i+1}<i+1}} \frac{2^{\ell}}{\left(y_{j_{\tau_{1}}}-y_{j_{\tau_{2}}}\right)^{2} \times \cdots \times\left(y_{j_{\tau_{i}}}-y_{j_{\tau_{i+1}}}\right)^{2}} .
\end{aligned}
$$

Combining these two, in order to get (B.5), it remains to show

$$
\begin{aligned}
& \sum_{\tau \in \mathfrak{J}_{i+1}^{\ell}} \frac{2^{\ell}}{\left(y_{j_{\tau_{1}}}-y_{j_{\tau_{2}}}\right)^{2} \times \cdots \times\left(y_{j_{\tau_{i}}}-y_{j_{\tau_{i+1}}}\right)^{2}} \\
= & \sum_{\sigma \in \mathfrak{J}_{i}^{\ell-1}} \frac{2^{\ell}}{\left(y_{j_{\sigma_{1}}}-y_{j_{\sigma_{2}}}\right)^{2} \times \cdots \times\left(y_{j_{\sigma_{i-2}}}-y_{j_{\sigma_{i-1}}}\right)^{2} \times\left(y_{j_{i+1}}-y_{j_{\sigma_{i}}}\right)^{2}} .
\end{aligned}
$$

To derive (B.8), we define $T: \mathfrak{J}_{i+1}^{\ell} \longrightarrow \mathfrak{J}_{i}^{\ell-1}$ in the following way. For $\tau \in \mathfrak{J}_{i+1}^{\ell}$, we must have $\tau_{2 k}=i+1$ for some $1 \leq k \leq \ell$. We set $\sigma_{j}=\tau_{j}$, for $j \leq 2 k-2$; we set $\sigma_{j}=\tau_{j+2}$, for $2 k-1 \leq j \leq 2 \ell-2$; and we set $\sigma_{i}=\tau_{2 k-1}$. This defines the map $T(\tau)=\sigma$. We argue that $T$ is a bijection. For any $\sigma \in \mathfrak{J}_{i}^{\ell-1}$, we can define $\tau$ as follows: $\left\{\left\{\tau_{1}, \tau_{2}\right\}, \ldots,\left\{\tau_{i}, \tau_{i+1}\right\}\right\}=\left\{\left\{\sigma_{1}, \sigma_{2}\right\}, \ldots,\left\{\sigma_{i-2}, \sigma_{i-1}\right\},\left\{\sigma_{i}, i+1\right\}\right\}$ such that $\tau_{1}<\tau_{3}<\cdots<\tau_{i}, \tau_{2 j-1}<\tau_{2 j}$ for $j \leq \ell$. Then we have $\tau \in \mathfrak{J}_{i+1}^{\ell}$. This implies $T$ is a bijection, and gives (B.8). Hence, we complete the proof of (B.5) for odd $i$, and complete the proof of (B.5).

Eq. (B.5) gives (B.2) for $\# K=i+1$ and completes the induction. Hence, Eq. (B.2) holds for all $K \subset J$. Taking $K=J$ in (B.2), we obtain (B.1). This completes the proof.

\section{References}

[ALS20] Juhan Aru, Titus Lupu, and Avelio Sepúlveda. The first passage sets of the 2d Gaussian free field: convergence and isomorphism. Comm. Math. Phys., 375(3):1885-1929, 2020. 


\section{Crossing probabilities in metric graph GFF}

\section{MR-4091511}

[BB03] Michel Bauer and Denis Bernard. Conformal field theories of stochastic Loewner evolutions. Comm. Math. Phys., 239(3):493-521, 2003. MR-2000927

[BB04] Michel Bauer and Denis Bernard. Conformal transformations and the SLE partition function martingale. Ann. Henri Poincaré, 5(2):289-326, 2004. MR-2057676

[BBK05] Michel Bauer, Denis Bernard, and Kalle Kytölä. Multiple Schramm-Loewner evolutions and statistical mechanics martingales. J. Stat. Phys., 120(5-6):1125-1163, 2005. MR-2187598

[Dub06] Julien Dubédat. Euler integrals for commuting SLEs. J. Stat. Phys., 123(6):1183-1218, 2006. MR-2253875

[Dub07] Julien Dubédat. Commutation relations for Schramm-Loewner evolutions. Comm. Pure Appl. Math., 60(12):1792-1847, 2007. MR-2358649

[Dub09] Julien Dubédat. SLE and the free field: partition functions and couplings. J. Amer. Math. Soc., 22(4):995-1054, 2009. MR-2525778

[Dub15] Julien Dubédat. SLE and Virasoro representations: fusion. Comm. Math. Phys., 336(2):761-809, 2015. MR-3322386

[DWW20] Jian Ding, Mateo Wirth, and Hao Wu. Crossing estimates from metric graph and discrete GFF. arXiv:2001.06447, 2020.

[FK04] Roland Friedrich and Jussi Kalkkinen. On conformal field theory and stochastic Loewner evolution. Nuclear Phys. B, 687(3):279-302, 2004. MR-2059141

[FK15] Steven M. Flores and Peter Kleban. A solution space for a system of null-state partial differential equations: Part 2. Comm. Math. Phys., 333(1):435-481, 2015. MR-3294955

[FSKZ17] S. M. Flores, J. J. H. Simmons, P. Kleban, and R. M. Ziff. A formula for crossing probabilities of critical systems inside polygons. J. Phys. A, 50(6):064005, 91, 2017. MR-3606709

[KKP19] Alex Karrila, Kalle Kytölä, and Eveliina Peltola. Conformal blocks, $q$-combinatorics, and quantum group symmetry. Ann. Inst. Henri Poincaré D, 6(3):449-487, 2019. MR-4002673

[KKP20] Alex Karrila, Kalle Kytölä, and Eveliina Peltola. Boundary correlations in planar LERW and UST. Comm. Math. Phys., 376(3):2065-2145, 2020. MR-4104543

[KP16] Kalle Kytölä and Eveliina Peltola. Pure partition functions of multiple SLEs. Comm. Math. Phys., 346(1):237-292, 2016. MR-3528421

[KW11] Richard W. Kenyon and David B. Wilson. Boundary partitions in trees and dimers. Trans. Amer. Math. Soc., 363(3):1325-1364, 2011. MR-2737268

[Lup16] Titus Lupu. From loop clusters and random interlacements to the free field. Ann. Probab., 44(3):2117-2146, 2016. MR-3502602

[MS16] Jason Miller and Scott Sheffield. Imaginary geometry I: Interacting SLEs. Probab. Theory Related Fields, 164(3-4):553-705, 2016. MR-3477777

[Pel19] Eveliina Peltola. Toward a conformal field theory for Schramm-Loewner evolutions. J. Math. Phys., 60(10):103305, 39, 2019. MR-4021824

[Pel20] Eveliina Peltola. Basis for solutions of the Benoit \& Saint-Aubin PDEs with particular asymptotics properties. Ann. Inst. Henri Poincaré D, 7(1):1-73, 2020. MR-4071342

[PW18] Eveliina Peltola and Hao Wu. Crossing probabilities of multiple Ising interfaces. arXiv:1808.09438, 2018.

[PW19] Eveliina Peltola and Hao Wu. Global and local multiple SLEs for $\kappa \leq 4$ and connection probabilities for level lines of GFF. Comm. Math. Phys., 366(2):469-536, 2019. MR-3922531

[RS05] Steffen Rohde and Oded Schramm. Basic properties of SLE. Ann. of Math. (2), 161(2):883924, 2005. MR-2153402

[She07] Scott Sheffield. Gaussian free fields for mathematicians. Probab. Theory Related Fields, 139(3-4):521-541, 2007. MR-2322706

[SS09] Oded Schramm and Scott Sheffield. Contour lines of the two-dimensional discrete Gaussian free field. Acta Math., 202(1):21-137, 2009. MR-2486487

[SS13] Oded Schramm and Scott Sheffield. A contour line of the continuum Gaussian free field. Probab. Theory Related Fields, 157(1-2):47-80, 2013. MR-3101840 
[Wu20] Hao Wu. Hypergeometric SLE: conformal Markov characterization and applications. Comm. Math. Phys., 374(2):433-484, 2020. MR-4072221

[WW17] Menglu Wang and Hao Wu. Level lines of Gaussian free field I: zero-boundary GFF. Stochastic Process. Appl., 127(4):1045-1124, 2017. MR-3619265

Acknowledgments. We acknowledge Jian Ding, Titus Lupu, and Mateo Wirth for helpful discussion on GFF. We acknowledge Eveliina Peltola for stimulating discussion about partition functions for multiple SLEs. We acknowledge the referee for careful comments which improved the presentation and provided deep insight into CFT. 